Louisiana State University

LSU Digital Commons

Faculty Publications

Department of Mathematics

3-1-2016

\title{
Excluded-Minor Characterization of Apex-Outerplanar Graphs
}

Guoli Ding

Louisiana State University

Stan Dziobiak

Recinto Universitario de Mayagüez

Follow this and additional works at: https://digitalcommons.Isu.edu/mathematics_pubs

\section{Recommended Citation}

Ding, G., \& Dziobiak, S. (2016). Excluded-Minor Characterization of Apex-Outerplanar Graphs. Graphs and Combinatorics, 32 (2), 583-627. https://doi.org/10.1007/s00373-015-1611-9

This Article is brought to you for free and open access by the Department of Mathematics at LSU Digital Commons. It has been accepted for inclusion in Faculty Publications by an authorized administrator of LSU Digital Commons. For more information, please contact ir@lsu.edu. 
Louisiana State University LSU Digital Commons

2011

\section{Excluded-minor characterization of apex- outerplanar graphs}

Stanislaw Dziobiak

Louisiana State University and Agricultural and Mechanical College, standzio@math.lsu.edu

Follow this and additional works at: https://digitalcommons.lsu.edu/gradschool_dissertations

Part of the Applied Mathematics Commons

\section{Recommended Citation}

Dziobiak, Stanislaw, "Excluded-minor characterization of apex-outerplanar graphs" (2011). LSU Doctoral Dissertations. 3102. https://digitalcommons.lsu.edu/gradschool_dissertations/3102

This Dissertation is brought to you for free and open access by the Graduate School at LSU Digital Commons. It has been accepted for inclusion in LSU Doctoral Dissertations by an authorized graduate school editor of LSU Digital Commons. For more information, please contactgradetd@lsu.edu. 


\title{
EXCLUDED-MINOR CHARACTERIZATION OF APEX-OUTERPLANAR GRAPHS
}

\author{
A Dissertation \\ Submitted to the Graduate Faculty of the \\ Louisiana State University and \\ Agricultural and Mechanical College \\ in partial fulfillment of the \\ requirements for the degree of \\ Doctor of Philosophy \\ in
}

The Department of Mathematics

by

Stanislaw Dziobiak

B.S. in Computer Science, Cornell University, 2002

M.Eng. in Computer Science, Cornell University, 2003

M.S. in Mathematics, Louisiana State University, 2007

August 2011 


\section{Acknowledgments}

"Lord grant that I shall never seek so much to be consoled, as to console; to be understood, as to understand; or to be loved, as to love, with all my heart."

(Prayer of St. Francis)

This work would not have been possible without my advisor Professor Guoli Ding. Thank you sir for introducing me to the beautiful field of graph theory, for suggesting this and other interesting problems, for always pointing me in the right direction, for your patience, encouragement, and inspiration.

I am forever indebted to my father Wiesław Dziobiak who has always been my role model, taught me everything I know about life, and always sacrificed himself for the benefit of our family.

I would like to thank all of my mathematics teachers who have motivated and inspired me over the years, among them: Wiesław Dziobiak, Włodzimierz Obrembski, René Torres, Ravi Ramakrishna, Dexter Kozen, Bogdan Oporowski, James Oxley, and Guoli Ding.

I would like to thank the members of my final exam committee, professors: Guoli Ding, Richard Litherland, Bogdan Oporowski, James Oxley, Robert Perlis, and Sudipta Sarangi, for their thoughtful comments and suggestions regarding this dissertation.

I would like to thank all of the mathematics faculty, staff, and graduate students at LSU for making my graduate school experience an enjoyable and memorable one.

Finally, I would like to thank my all of my family, friends, and colleagues for their constant support and reasons only they understand.

This dissertation is dedicated to the memory of my mother Teresa Dziobiak (1952 - 2011). Thank you for your unconditional love. 


\section{Table of Contents}

Acknowledgments $\ldots \ldots \ldots \ldots \ldots \ldots \ldots \ldots \ldots \ldots \ldots \ldots \ldots \ldots \ldots \ldots \ldots \ldots$ ii

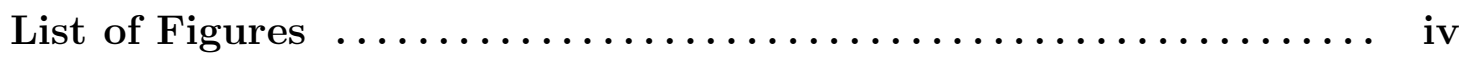

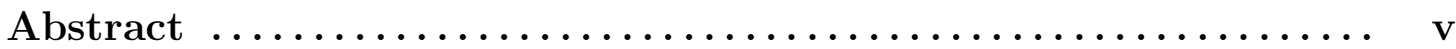

Chapter 1: Introduction $\ldots \ldots \ldots \ldots \ldots \ldots \ldots \ldots \ldots \ldots \ldots, 1$

1.1 Overview . . . . . . . . . . . . . . . . . . 1

1.2 Preliminaries . . . . . . . . . . . . . . . . . . . 5

1.3 Graph Minors . . . . . . . . . . . . . . . . . . . . . . . . . . 9 9

1.4 Related Results . . . . . . . . . . . . . . . . . . . . . . . . 12

1.5 Main Result . . . . . . . . . . . . . . . . . . . . . . . . 15

Chapter 2: Key Lemma ........................... 16

2.1 Definitions . . . . . . . . . . . . . . . . . . 16

2.2 Starting Point . . . . . . . . . . . . . . . . . . . . . . 17

2.3 Key Lemma . . . . . . . . . . . . . . . . . . . . . . . . . . . 19

Chapter 3: Connectivity Two $\ldots \ldots \ldots \ldots \ldots \ldots \ldots \ldots \ldots \ldots \ldots .24$

3.1 Roadmap . . . . . . . . . . . . . . . . . . . . 24

3.2 Case 1: Both Sides Non-Outerplanar . . . . . . . . . . . . . . 24

3.3 Case 2: One Side Outerplanar . . . . . . . . . . . . . . . 28

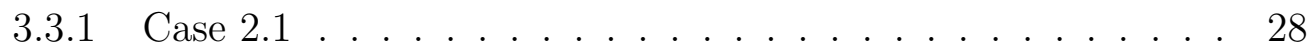

3.3 .2 Case $2.2 \ldots \ldots \ldots \ldots \ldots$

Chapter 4: Connectivity Three $\ldots \ldots \ldots \ldots \ldots \ldots \ldots \ldots \ldots \ldots \ldots 66$

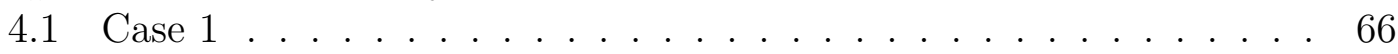

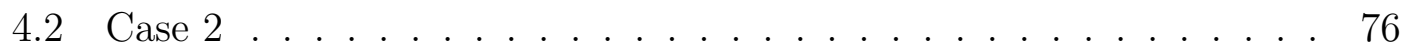

References $\ldots \ldots \ldots \ldots \ldots \ldots \ldots \ldots \ldots \ldots \ldots \ldots \ldots \ldots \ldots \ldots \ldots \ldots .61$

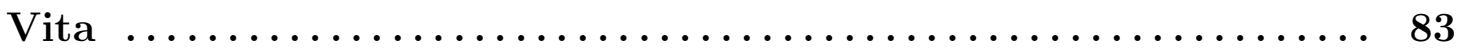




\section{List of Figures}

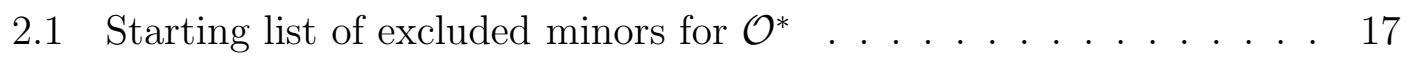

$2.2 \quad K_{4}$ and $K_{2,3}$ 's with prescribed vertices $x$ and $y \ldots \ldots . . . . . . .19$

$2.3 \quad P_{2}$ and $C_{4} \ldots \ldots \ldots \ldots \ldots \ldots \ldots$

$3.1 \mathcal{T}$ family . . . . . . . . . . . . . . . . . . 27

$3.2 \mathcal{G}$ family . . . . . . . . . . . . . . . . . . . 32

$3.3 \mathcal{J}$ family . . . . . . . . . . . . . . . . . . . . . 32

$3.4 \mathcal{H}$ family . . . . . . . . . . . . . . . . . . . . 46

$3.5 \mathcal{Q}$ family . . . . . . . . . . . . . . . . . . 51 


\section{Abstract}

It is well known that the class of outerplanar graphs is minor-closed and can be characterized by two excluded minors: $K_{4}$ and $K_{2,3}$. The class of graphs that contain a vertex whose removal leaves an outerplanar graph is also minor-closed. We provide the complete list of 57 excluded minors for this class. 


\section{Chapter 1}

\section{Introduction}

\subsection{Overview}

One of the deepest and by many considered the most important works in graph theory is the Graph Minor Theory developed by Neil Robertson and Paul Seymour, and joined in the later stages by Robin Thomas. One of the main results of this work is the proof of Wagner's Conjecture [15] that finite graphs are well-quasiordered under the minor relation, namely, for every infinite sequence of graphs, at least one graph is a minor of another (where a graph is a minor of another if the first can be obtained from a subgraph of the second by contracting edges). An equivalent formulation of this result is the following:

Theorem 1.1. [15] Any minor-closed class of graphs can be characterized by a finite number of excluded minors.

A minor-closed class of graphs is a class closed under the operation of taking minors; also, a graph $G$ is an excluded minor or an obstruction of a class $\mathcal{C}$ if $G$ is not a member of $\mathcal{C}$, but every proper minor of $G$ is; the complete (finite) list of excluded minors of a class $\mathcal{C}$ is called its obstruction set and is denoted by $\mathbf{o b}(\mathcal{C})$. This work took more than 21 years to publish in a series of 23 long papers, and it led to entirely new concepts and a new way of looking at graph theory. At the core of this monumental project lies a powerful theorem capturing the general structure of graphs that do not contain a fixed minor [14]. The rough formulation of this theorem is as follows: 
Theorem 1.2. If a minor-closed class of graphs does not contain all graphs, then every graph in it is glued together in a tree-like fashion from graphs that can be nearly embedded in a fixed surface.

For the precise formulation of this theorem see [5]. Recently, a number of beautiful results that use this structural result have appeared (for a survey, see [10]).

One of the simplest structures satisfying the conclusion of Theorem 1.2 is that of apex-planar graphs. A graph is apex-planar if it contains a vertex whose removal leaves a planar graph. The class of apex-planar graphs is the motivating class behind our research. It arises in two of the hardest and most famous conjectures in graph theory: Hadwiger's Conjecture and Jorgensen's Conjecture.

First, Hadwiger's Conjecture [8] states that graphs with no $K_{n}$-minor can be colored with at most $n-1$ colors (a coloring of a graph $G=(V(G), E(G)$ ) is an assignment of a color to each vertex $v \in V(G)$ such that no two adjacent vertices are assigned the same color). While the statement has been shown to be true for $n \leqslant 6$ (the case $n=5$ is, according to [20], equivalent to the Four Color Theorem [12], while the case $n=6$ was proved in [16]), it remains open for $n \geqslant 7$. To prove Hadwiger's Conjecture for $n=6$, the authors of [16] show (without assuming the Four Color Theorem) that every minimal counterexample to Hadwiger's conjecture for $n=6$ is apex-planar. With this theorem in place, Hadwiger's conjecture for $n=6$ follows immediately since, by the Four Color Theorem, apex-planar graphs are 5-colorable, and so no counterexample exists.

Secondly, Jorgensen's Conjecture (which implies Hadwiger's Conjecture for $n=$ 6) states that every 6 -connected graph with no $K_{6}$-minor is apex-planar. The fact that the conjecture holds for large graphs has been shown by Thomas and Norin (see Theorem 1.14). 
In fact, it has been a long-standing problem to find an explicit excluded-minor characterization for the class of apex-planar graphs [1]. It must be mentioned that even though Theorem 1.1 assures us that a given minor-closed class of graphs has finitely many minimal excluded minors, the proof is not constructive and for most minor-closed classes, including apex-planar graphs, we do not know how to obtain its finite obstruction set, since there are no general techniques for doing this (for an interesting result on the actual computability of this problem, see [2]). Besides providing an elegant characterization of a minor-closed class $\mathcal{C}$, the knowledge of the complete list of excluded minors (the obstruction set) of $\mathcal{C}$ is very useful as it allows for efficient (polynomial-time) testing for membership in $\mathcal{C}$. This is due to the following algorithmic implication of Robertson and Seymour:

Theorem 1.3. [13] For every fixed graph $H$, there exists an $O\left(n^{3}\right)$ algorithm for deciding if a given graph of order $n$ contains $H$ as a minor.

Therefore, to test whether a given graph belongs to $\mathcal{C}$, one simply checks whether it contains any of the excluded minors of $\mathcal{C}$ as a minor. Since the obstruction set of $\mathcal{C}$ is finite, the number of such checks is a constant (that depends only on $\mathcal{C}$ ).

Because finding the complete explicit list of excluded minors for the class of apex-planar graphs has been well known to be very hard, we have tried to find an excluded-minor characterization of a very similar class, namely that of apexouterplanar graphs, in hopes that it will shed light on the original problem. A graph is apex-outerplanar if it contains a vertex whose removal leaves an outerplanar graph; and an outerplanar graph is one with no $K_{4^{-}}$or $K_{2,3}$-minor.

Our general technique was to narrow down the possible structure of an excluded minor for this class. After quickly finding an initial set of excluded minors, which included $K_{5}$ and the octahedron, we were able to conclude that the connectivity 
of the remaining excluded minors must be 2 or 3 using the following theorem of Halin and Jung [7]: if $\delta(G) \geqslant 4$, then $G$ contains $K_{5}$ or the octahedron as a minor.

The farther we got in the proof, the more the structure of the excluded minors tightened. Eventually, after a long struggle, we generated the proof of the complete list of 57 excluded minors. Our list has been confirmed by Galen E. Turner [19], who computed this list with the aid of computers, but without proof of its completeness. Three of the graphs on our list are simply disjoint copies of $K_{4}$ 's and $K_{2,3}$ 's; four of them are 3-connected: $K_{5}, K_{3,3}$, the cube, and the octahedron (Platonic solids); and the remaining fifty have connectivity 2 , twenty of which, unsurprisingly, turn out to be triangular arrangements of $K_{4}$ 's and $K_{2,3}$. It is exactly the analogous arrangements of $K_{5}$ 's and $K_{3,3}$ 's that are excluded minors for the original class of apex-planar graphs, thus at least, giving us a lower bound on the number of such graphs, and shedding some light on the original open problem.

Our problem also falls within a more general framework. Let $\mathcal{C}$ be a minorclosed class of graphs, and let $\mathcal{C}^{*}$ be the class of graphs that contain a vertex whose removal leaves a graph in $\mathcal{C}$. Hence, clearly $\mathcal{C} \subseteq \mathcal{C}^{*}$, and it is easy to check that $\mathcal{C}^{*}$ is also minor-closed (see Observation 1.12). Consider the following problem: given a minor-closed class $\mathcal{C}$ and $\mathbf{o b}(\mathcal{C})$, find $\mathbf{o b}\left(\mathcal{C}^{*}\right)$. Adler, et. al [2] showed that this problem is computable. That is, they show that there exists an algorithm that given $\mathbf{o b}(\mathcal{C})$ computes $\mathbf{o b}\left(\mathcal{C}^{*}\right)$. However, their result is purely existential, it does not yield an explicit algorithm.

In this dissertation, we find the explicit list $\mathbf{o b}\left(\mathcal{O}^{*}\right)$ given $\mathbf{o b}(\mathcal{O})=\left\{K_{4}, K_{2,3}\right\}$. Our result is the first one in literature of this kind, in the sense that $\mathbf{o b}\left(\mathcal{C}^{*}\right)$ has not been found for any other non-trivial class $\mathcal{C}$. 


\subsection{Preliminaries}

We begin by introducing some standard graph theoretical terminology that will be use throughout the dissertation. For a more complete treatment please refer to [3], [4], or [22].

A graph $G$ is an ordered pair $(V(G), E(G))$ consisting of a finite set $V(G)$ of vertices and a finite multiset $E(G)$ of edges, disjoint from $V(G)$, whose elements are unordered pairs of (not necessarily distinct) elements of $V(G)$. If $u, v \in V(G)$ and $e=\{u, v\} \in E(G)$ then $u$ and $v$ are called the endpoints of the edge $e$, and $e$ denoted simply as $u v$. An edge is said to be incident with each of its endpoints and vice versa. If $u v \in E(G)$, then vertices $u$ and $v$ are said to be adjacent, or neighbors. Two edges are said to be adjacent if they have a common endpoint. An edge with identical endpoints is called a loop, and two edges incident with the same pair of distinct endpoints are called parallel edges. A graph is simple if it has no loops nor parallel edges. To avoid confusion, we refer to graphs with possible loops and parallel edges as multigraphs. A simplification of a multigraph $G$ is the simple graph obtained from $G$ by deleting (see definition below) all loops and parallel edges. All of the graphs considered in this dissertation are assumed to be simple (see Section 1.5 for the reason why).

If $G_{1}$ and $G_{2}$ are two graphs, then $G_{1} \mid G_{2}$ denotes the disjoint union of $G_{1}$ and $G_{2}$, that is, a graph $H$ such that $V(H)=V\left(G_{1}\right) \cup V\left(G_{2}\right)$ and $E(H)=E\left(G_{1}\right) \cup E\left(G_{2}\right)$, where both set unions are disjoint.

Let $v \in V(G)$, then the set of neighbors of $v$ in $G$ is called the neighborhood of $v$ and is denoted as $N_{G}(v)$, or simply as $N(v)$ if the context is understood. Also, the number of edges of $G$ incident with $v$ is the degree of $v$ in $G$, and denoted as $\operatorname{deg}_{G}(v)$, or simply as $\operatorname{deg}(v)$. This is equal to $|N(v)|$ by our assumption that $G$ is simple. The number $\delta(G):=\min \left\{\operatorname{deg}_{G}(v) \mid v \in V(G)\right\}$ is called the minimum degree 
of $G$, and the number $\Delta(G):=\max \left\{\operatorname{deg}_{G}(v) \mid v \in V(G)\right\}$ is called the maximum degree of $G$.

Two graphs $G=(V(G), E(G))$ and $H=(V(H), E(H))$ are isomorphic, denoted as $G \cong H$, if there exists a bijection $\phi: V(G) \rightarrow V(H)$ with $u v \in E(G)$ if and only if $\phi(u) \phi(v) \in E(H)$. A subgraph of a graph $G$ is a graph $H$ such that $V(H) \subseteq V(G)$ and $E(H) \subseteq E(G)$. In this case, we say that $G$ contains $H$, and write $H \subseteq G$. $H$ is a proper subgraph of $G$ if $H \subseteq G$ and $H \neq G$. If $H \subseteq G$ and $V(H)=V(G)$, then $H$ is said to be a spanning subgraph of $G$. If $H \subseteq G$ and $H$ contains all the edges of $G$ whose endpoints belong to $V(H)$, then $H$ is an induced subgraph of $G$, and if $S=V(H)$, then we write $H=G[S]$ and say that $H$ is the subgraph of $G$ induced by $S$.

Alternatively, we may think of a subgraph $H \subseteq G$ as being obtained from $G$ by deleting edges and/or vertices (and all of their incident edges). The subgraph of $G$ obtained by deleting edge $e$ will be denoted by $G \backslash e$. Similarly, if $F \subseteq E(G)$ then $G \backslash F$ denotes the subgraph of $G$ obtained by deleting all the edges of $F$; if $v \in V(G)$ then $G-v$ denotes the subgraph of $G$ obtained by deleting vertex $v$ and all of its incident edges, and if $W \subseteq V(G)$ then $G-W$ denotes the subgraph of $G$ obtained by deleting all of the vertices in $W$ and all of their incident edges. Note that $G[W]=G-\bar{W}$, where $\bar{W}$ is the complement of $W$ in $V(G)$.

A complete graph or clique is a simple graph in which every pair of vertices is adjacent. A clique on $n$ vertices is denoted by $K_{n}$. A graph $G$ is bipartite if $V(G)$ is the union of two disjoint sets $X$ and $Y$ such that each edge of $G$ has one endpoint in $X$ and the other in $Y$. If, furthermore, every vertex in $X$ is adjacent to every vertex in $Y$, then $G$ is called a complete bipartite graph, and if $m:=|X|$, and $n:=|Y|$, then $G$ is denoted by $K_{m, n}$. 
A path is a graph $P=(V(P), E(P))$ with $V(P)=\left\{v_{1}, v_{2}, \ldots, v_{k+1}\right\}$ where $k \geqslant 0$, and $E(P)=\left\{v_{1} v_{2}, v_{2} v_{3}, \ldots, v_{k} v_{k+1}\right\}$. The length of $P$ is $k$, and $P$ is also denoted by $P_{k}$. If $u:=v_{1}$ and $v:=v_{k+1}$, then $P$ is called a $u v$-path. The set $\left\{v_{2}, v_{3}, \ldots, v_{k}\right\}$ is called the interior of $P$ and is denoted by $\operatorname{int}(P)$. Two paths $P$ and $Q$ are said to be internally disjoint if their interiors are disjoint. Similarly, a cycle is a graph $C=(V(C), E(C))$ with $V(C)=\left\{v_{1}, v_{2}, \ldots, v_{k}\right\} k \geqslant 3$, and $E(C)=\left\{v_{1} v_{2}, v_{2} v_{3}, \ldots, v_{k-1} v_{k}, v_{k} v_{1}\right\}$. The length of $C$ is $k$, and $C$ is also denoted by $C_{k}$. An edge $e \notin E(C)$ with both endpoints in $V(C)$ is called a chord of $C$. Let $C=u_{1}, u_{2}, \ldots u_{n}, u_{1}$ be a cycle with vertices listed in the clockwise order around $C$. We denote by $C\left[u_{i}, u_{j}\right]$ the set $\left\{u_{i}, u_{i+1}, \ldots, u_{j}\right\}$ if $i \leqslant j$, or the set $\left\{u_{i}, u_{i+1}, \ldots, u_{n}, u_{1}, \ldots, u_{j}\right\}$ if $i>j$. Similarly, $C\left[u_{i}, u_{j}\right):=C\left[u_{i}, u_{j}\right]-\left\{u_{j}\right\}$, $C\left(u_{i}, u_{j}\right]:=C\left[u_{i}, u_{j}\right]-\left\{u_{i}\right\}$, and $C\left(u_{i}, u_{j}\right):=C\left[u_{i}, u_{j}\right]-\left\{u_{i}, u_{j}\right\}$. Also, if $P=$ $u_{1}, u_{2}, \ldots u_{n}$ is a path, then we define $P\left[u_{i}, u_{j}\right], P\left[u_{i}, u_{j}\right), P\left(u_{i}, u_{j}\right]$, and $P\left(u_{i}, u_{j}\right)$ analogously, and by $\operatorname{int}(P)$ we denote $P\left(u_{1}, u_{n}\right)$.

A graph $G$ is connected if for each pair of distinct vertices $u, v \in V(G), G$ contains a $u v$-path. If $G$ is not connected, then we say that it is disconnected. A connected graph that contains no cycles is called a tree. A vertex of degree 1 in a tree $T$ is called a leaf of $T$. A maximal connected subgraph of $G$ is called a component of $G$. Let $u$ and $v$ be non-adjacent vertices of $G$. A $u v$-vertex cut is a set $S \subseteq V(G)-\{u, v\}$ such that $u$ and $v$ belong to different components of $G-S$. In this case we say that $S$ separates $u$ and $v$. A vertex cut separating some pair of non-adjacent vertices is simply called a vertex cut of $G$, and one with $k$ elements is called a $k$-vertex cut, or simply a $k$-cut. The only element in a 1 -vertex cut is called a cut vertex. $G$ is said to be $k$-connected if $|V(G)| \geqslant k+1$ and every vertex cut has at least $k$ vertices. The connectivity of $G$, denoted by $\kappa(G)$, is the maximum $k$ such that $G$ is $k$-connected (if $G$ is not a clique, this is equal to the minimum 
size of a vertex cut). By convention, $\kappa(G)=0$ if and only if $G$ is disconnected or $G=K_{1}$, and $\kappa\left(K_{n}\right)=n-1$ for all $n \geqslant 1$.

The following theorem is a classical result of Menger from 1927, which can be found in [3].

Theorem 1.4 (Menger's Theorem). Let $G$ be a graph and $u$ and $v$ two nonadjacent vertices of $G$. Then the maximum number of pairwise internally disjoint uv-paths is equal to the minimum size of a uv-vertex cut.

The following definition has been adopted from [3]. Let $H$ be a proper subgraph of a connected graph $G$. The set $E(G) \backslash E(H)$ may be partitioned into classes as follows.

- For each component $F$ of $G-V(H)$, there is a class consisting of the edges of $F$ together with the edges joining $F$ to $H$.

- Each remaining edge $e$ (that is, one which has both enpoints in $V(H)$ ) defines a singleton class $\{e\}$.

The subgraphs of $G$ induced by these classes are the bridges of $H$, or $H$-bridges. It follows immediately from the definition that bridges of $H$ can intersect only in vertices of $H$, and that any two vertices of a bridge of $H$ are connected by a path in the bridge that is internally disjoint from $H$. For a bridge $B$ of $H$, the elements of $V(B) \cap V(H)$ are called the feet of $B$, and for a set $W \subseteq V(H)$, we say that the feet attach to $W$ if $V(B) \cap V(H) \subseteq W$. The remaining vertices of $B$ are its internal vertices. A bridge is trivial if it has no internal vertices (that is, if it is of the second type). In a connected graph, every bridge has at least one foot; and in a 2-connected graph, every bridge has at least two feet.

A block of a connected graph $G$ is a maximal 2-connected subgraph of $G$, or a $K_{2}$-subgraph of $G$, one of whose vertices has degree 1 in $G$, or both of whose 
vertices are cut vertices in $G$. We note that two distinct blocks in $G$ share at most one vertex since otherwise their union would be 2-connected. The block tree of $G$ is a tree $T$ whose vertex set is the disjoint union of the set of blocks of $G$ and the set of cut vertices of $G$. The only edges in $T$ are those that join cut vertices of $G$ to blocks that contain them. The fact that the block tree of a connected graph is indeed a tree is non-trivial, but is an easy consequence of the definition and can be found in [4].

A planar embedding of a graph $G$ is a drawing of $G$ in the plane where the vertices are represented by points, the edges by simple curves joining the endpoints, and the edges intersect only at their endpoints. Graph $G$ is said to be planar or embeddable in the plane if it has a planar embedding. A plane graph is a particular planar embedding of a planar graph. The faces of a plane graph are the maximal regions (open sets) of the plane that are disjoint from the embedding. Each plane graph has exactly one unbounded face, called the outer face or the infinite face. The boundary of a face $f$ is the boundary of the open set $f$ in the usual topological sense. A face is said to be incident with the vertices and edges in its boundary.

\subsection{Graph Minors}

To contract an edge $e=u v \in E(G)$ is to delete the edge $e$ and identify its endpoints $u$ and $v$. We denote the resulting graph by $G / e$. Because we are only considering simple graphs, we implicitly assume that such an operation is immediately followed by the deletion of a parallel edge, if necessary. More precisely, $G / e$ is the simple graph obtained by deleting vertices $u$ and $v$, and adding a new vertex $w$ whose neighborhood is the set $\left(N_{G}(u) \cup N_{G}(u)\right)-\{u, v\}$. Similarly, if $F \subseteq E(G)$ then $G / F$ denotes the graph obtained by contracting the edges of $F$ one by one in any order (the result is independent of the order). 
Given graphs $H$ and $G, H$ is a minor of $G$, denoted by $H \leqslant_{m} G$, or $G \geqslant_{m} H$, if $H$ can be obtained from a subgraph of $G$ by contracting edges. Equivalently, $H \leqslant_{m} G$ if $H$ can be obtained from $G$ by any sequence of the following operations: deleting vertices, deleting edges, and contracting edges. Also, a useful alternative definition of a minor is the following.

Definition 1.5. Let $G$ be a graph, and consider a partition $\left(V_{0}, V_{1}, \ldots, V_{k}\right)$ of $V$ such that $G\left[V_{i}\right]$ is connected for $i=1, \ldots, k$. Let $H$ be the graph obtained from $G$ by deleting $V_{0}$ and contracting each induced subgraph $G\left[V_{i}\right]$ to a single vertex. Then any spanning subgraph $F$ of $H$ is said to be a minor of $G$.

Let $G$ be a graph, and $e=u v \in E(G)$. To subdivide the edge $e$ is to delete $e$, add a new vertex $w$, and two new edges $u w$ and $w v$. Any graph obtained from a graph $G$ by a sequence of edge subdivisions is called a subdivision of $G$, or $G$-subdivision.

Clearly, if $G$ contains an $H$-subdivision (in literature $H$ is called a topological minor of $G$ ), then $G \geqslant_{m} H$. The converse also holds if $\Delta(H) \leqslant 3$ as shown in Lemma 1.6.

Lemma 1.6. If $H \leqslant{ }_{m} G$ and $\Delta(H) \leqslant 3$, then $G$ contains an $H$-subdivision.

Proof. Let $H \leqslant_{m} G$. Then, $G$ has a vertex partition $\left(V_{0}, V_{1}, \ldots, V_{k}\right)$ as in Definition 1.5 , and we may assume that $H$ is a spanning subgraph of $H^{\prime}$, where $H^{\prime}$ is the graph obtained from $G$ by deleting $V_{0}$ and shrinking each induced subgraph $G\left[V_{i}\right]$ to a single vertex $v_{i}$. Then, since each $v_{i} \in V(H)$ is obtained by shrinking the corresponding connected subgraph $G\left[V_{i}\right]$ for some $i$, we may assume, without loss of generality, that each $G\left[V_{i}\right]$ is a tree. Furthermore, since $\Delta(H) \leqslant 3$, we have that at most three edges go out of each tree $G\left[V_{i}\right]$. Now, we may further assume that each $G\left[V_{i}\right]$ only has at most three leaves, namely the leaves that are incident with the edges going out of $G\left[V_{i}\right]$. Hence, we may assume that each $G\left[V_{i}\right]$ is just 
a subdivision of $K_{1, \ell}$, where $\ell \in\{1,2,3\}$. Hence, in the shrinking of each $G\left[V_{i}\right]$ to produce a vertex of $H$, the only contractions performed are unsubdivisions. Hence $G$ contains an $H$-subdivision.

A class $\mathcal{C}$ of graphs is minor-closed if for every $G \in \mathcal{C}$ all the minors of $G$ are also in $\mathcal{C}$. A minor-closed class is proper if it is not the class of all graphs. Some examples of proper minor-closed classes are: planar graphs, outerplanar graphs (see Section 1.5), series-parallel graphs (see [4]), graphs embeddable in a fixed surface (see [11]), linklessly embeddable graphs (see [17]), and graphs of tree-width bounded by a fixed constant (see [4]).

To see, for instance, that the class of planar graphs is minor-closed, it is sufficient to verify that for any planar graph, all of its single-edge deletions and all of its single-edge contractions are planar. So let $\Pi$ be a planar embedding of a planar graph $G$, and let $e=u v \in E(G)$. Clearly, by deleting the simple curve representing edge $e$ from $\Pi$, we obtain a planar embedding $\Pi^{\prime}$ of $G \backslash e$. Similarly, we can identify the points representing vertices $u$ and $v$ by sliding them along the simple curve representing $e$ in such a way that no edges intersect (except at the endpoints), thus obtaining a planar embedding $\Pi^{\prime \prime}$ of $G / e$.

For a minor-closed class $\mathcal{C}$, we let $\mathbf{o b}(\mathcal{C})$ denote its set of excluded minors, that is, minor-minimal graphs not in $\mathcal{C}$ (that is, graphs that are not in $\mathcal{C}$, but whose every proper minor is in $\mathcal{C})$. We call $\mathbf{o b}(\mathcal{C})$ the obstruction set of $\mathcal{C}$. It is clear that a graph $G$ belongs to $\mathcal{C}$ if and only if it has no minor in $\mathbf{o b}(\mathcal{C})$. Therefore the set $\mathbf{o b}(\mathcal{C})$ characterizes the class $\mathcal{C}$. Such a characterization is called the excludedminor characterization of $\mathcal{C}$. The following is a landmark result of Robertson and Seymour [15]. 
Theorem 1.7 (Graph Minor Theorem). For any minor-closed class $\mathcal{C}, \operatorname{ob}(\mathcal{C})$ is finite.

Unfortunately, the proof of the above theorem is non-constructive, and hence gives no method of finding $\mathbf{o b}(\mathcal{C})$. In fact, a fully constructive proof is impossible. Fellows and Langston [6] proved that there is no algorithm to compute the obstruction set of an arbitrary minor-closed class $\mathcal{C}$, represented as a Turing machine that enumerates the elements of $\mathcal{C}$. More precisely,

Theorem 1.8. The following problem is undecidable:

Given: Turing machine deciding a minor-closed class $\mathcal{C}$

Problem: Compute $\mathbf{o b}(\mathcal{C})$.

The importance of knowing $\mathbf{o b}(\mathcal{C})$ explicitly is that it allows for polynomial-time membership testing according to the following result of Robertson and Seymour [13].

Theorem 1.9. For any fixed graph $H$, there is an algorithm to determine whether a given n-vertex graph has $H$ as a minor in $O\left(n^{3}\right)$-time.

Corollary 1.10. For any minor-closed class $\mathcal{C}$, there is an algorithm to determine whether a given n-vertex graph $G$ belongs to $\mathcal{C}$ in $O\left(n^{3}\right)$-time.

The only drawback of the above theorem is that it is non-constructive. Its proof does not yield an explicit description of the algorithm, only knowledge of its existence. Moreover, the constant hidden in the $O\left(n^{3}\right)$ running-time is an enormous function of the number of vertices of $H$.

\subsection{Related Results}

A graph $G$ is apex-planar if there exists $v \in V(G)$ such that $G-v$ is planar. Let $\mathcal{P}$ and $\mathcal{P}^{*}$ denote the classes of planar and apex-planar graphs, respectively. 
The following is Wagner's reformulation of Kuratowski's famous characterization of planar graphs. For a proof, see [4].

Theorem 1.11. $\mathrm{ob}(\mathcal{P})=\left\{K_{5}, K_{3,3}\right\}$.

Let $\mathcal{C}$ be a minor-closed class of graphs, and let $\mathcal{C}^{*}$ be the class of graphs that contain a vertex whose removal leaves a graph in $\mathcal{C}$. Hence, clearly $\mathcal{C} \subseteq \mathcal{C}^{*}$, and it is easy to check that $\mathcal{C}^{*}$ is also minor-closed.

Observation 1.12. $\mathcal{C}^{*}$ is minor-closed.

Proof. Let $G \in \mathcal{C}^{*}$, and let $e \in E(G)$. It is sufficient to show that both $G \backslash e$ and $G / e$ belong to $\mathcal{C}^{*}$. Let $v \in V(G)$ be such that $G-v \in \mathcal{C}$. If $e \in E(G-v)$, then since $\mathcal{C}$ is minor-closed, it follows that $v$ is still apex in $G \backslash e$ and in $G / e$. Otherwise, $e$ is incident with $v$ in $G$. Let $x$ be the other endpoint of $e$. Then $(G \backslash e)-v=G-v \in \mathcal{C}$, and $(G / e)-v=(G-v)-x \in \mathcal{C}$. Hence, in both cases both $G \backslash e$ and $G / e \in \mathcal{C}^{*}$.

Consider the following problem: given a minor-closed class $\mathcal{C}$ and $\mathbf{o b}(\mathcal{C})$, find $\operatorname{ob}\left(\mathcal{C}^{*}\right)$. Adler, et. al [2] showed that this problem is computable. That is, they show that there exists an algorithm that given $\mathbf{o b}(\mathcal{C})$ computes $\mathbf{o b}\left(\mathcal{C}^{*}\right)$. However, their result is purely existential, it does not yield an explicit algorithm.

Let $\mathcal{O}$ and $\mathcal{O}^{*}$ denote the classes of outerplanar and apex-outerplanar graphs, respectively (see next section for definitions). In this dissertation, we find the explicit list $\mathbf{o b}\left(\mathcal{O}^{*}\right)$ given $\mathbf{o b}(\mathcal{O})=\left\{K_{4}, K_{2,3}\right\}$ (see next section). Our result is the first one in literature of this kind, in the sense that $\mathbf{o b}\left(\mathcal{C}^{*}\right)$ has not been found for any other class $\mathcal{C}$.

A related result was done by Wargo [21] for $\alpha$-outerplanar multigraphs, in which he characterizes the class in terms of 13 excluded minors. An $\alpha$-outerplanar multigraph is a multigraph $G$ which is not outerplanar such that, for some edge $\alpha$, both multigraphs $G \backslash \alpha$ and $G / \alpha$ are outerplanar. It is easy to check that the class of 
$\alpha$-outerplanar multigraphs is a proper subclass of apex-outerplanar multigraphs (see next section).

A long-standing related open problem has been to find $\mathbf{o b}\left(\mathcal{P}^{*}\right)$, that is the excluded minors for the class of apex-planar graphs [1] $\left(\mathcal{P}^{*}\right.$ is minor-closed by Observation 1.12). However, the problem is known to be very hard and is widely open. Even if we restrict the class $\mathcal{P}^{*}$ to 6 -connected graphs, it is still not known what the complete list of excluded minors is. This problem has only been solved partially for large graphs.

Jorgensen [9] made the following powerful conjecture (which actually implies the famous Hadwiger's Conjecture for $t=6$ ).

Conjecture 1.13. Every 6-connected graph with no $K_{6}$-minor is apex-planar.

The conjecture is also related to two important open problems in structural graph theory, namely those to characterize the structure of graphs that do not contain the Petersen graph as a minor, and those that do not contain a $K_{6}$-minor.

The conjecture says that if we impose the strong, extra condition of 6-connectedness on the class of apex-planar graphs, then the only excluded minor is $K_{6}$. For large graphs, Thomas and Norin [18] proved the following powerful theorem about the structure of graphs that do not contain a $K_{t}$-minor, which for the value $t=6$ implies Jorgensen's conjecture for large graphs.

Theorem 1.14. For every there exists $N_{t}$ such that for every $t$-connected graph $G \ngtr_{m} K_{t}$ on at least $N_{t}$ vertices, there exists $X \subseteq V(G)$ with $|X| \leqslant t-5$ such that $G-X$ is planar.

For the value $t=6$, this is exactly the statement of Jorgensen's conjecture for large graphs. 


\subsection{Main Result}

A graph $G$ is outerplanar if it has a planar embedding with all of its vertices incident to one common face. An outerplanar embedding of $G$ is a planar embedding of $G$ with all of its vertices incident to the outer face. Note that every outerplanar graph has an outerplanar embedding. It is easy to see that the class of outerplanar graphs is minor-closed by an argument similar to the one for planar graphs from Section 1.3. We say that a graph $G$ is apex-outerplanar if there exists $v \in V(G)$ such that $G-v$ is outerplanar. Such a vertex, if it exists, is called an apex vertex of $G$. It follows from these definitions that a multigraph is apex-outerplanar if and only if its simplification is apex-outerplanar. This is the reason why we only consider simple graphs in this dissertation.

The following theorem follows easily from Theorem 1.11 .

Theorem 1.15. ob $(\mathcal{O})=\left\{K_{4}, K_{2,3}\right\}$.

Equivalently, since $\Delta\left(K_{4}\right) \leqslant 3$ and $\Delta\left(K_{2,3}\right) \leqslant 3$, it follows by Lemma 1.6 that:

Theorem 1.16. $G$ is outerplanar if and only if it does not contain a $K_{4}$ - nor $K_{2,3}$-subdivision.

As corollary to Observation 1.12, we obtain that $\mathcal{O}^{*}$ is minor-closed. As such, it admits an excluded-minor characterization, and the goal of our work is to find $\operatorname{ob}\left(\mathcal{O}^{*}\right)$. We now state our main result. Let $\mathcal{S}$ be the set of graphs in Figure 2.1, $\mathcal{T}$ be the set of graphs in Figure 3.1, $\mathcal{G}$ be the set of graphs in Figure $3.2, \mathcal{J}$ be the set of graphs in Figure 3.3, $\mathcal{H}$ be the set of graphs in Figure 3.4, and $\mathcal{Q}$ be the set of graphs in Figure 3.5.

Theorem 1.17. A graph is apex-outerplanar if and only if it does not contain any of the 57 graphs in the set $\mathcal{S} \cup \mathcal{T} \cup \mathcal{G} \cup \mathcal{J} \cup \mathcal{H} \cup \mathcal{Q}$ as a minor. Equivalently, $\operatorname{ob}\left(\mathcal{O}^{*}\right)=\mathcal{S} \cup \mathcal{T} \cup \mathcal{G} \cup \mathcal{J} \cup \mathcal{H} \cup \mathcal{Q}$ 


\section{Chapter 2 \\ Key Lemma}

\subsection{Definitions}

We start with a few important definitions.

Definition 2.1. Let $G$ be a connected graph with cut-vertex $x$. A 1-separation of $G$ across $x$ is a pair of subgraphs $(L, R)$ of $G$ such that:

(1) $E(L) \cup E(R)=E(G)$;

(2) $V(L) \cup V(R)=V(G)$ and $V(L) \cap V(R)=\{x\}$.

Definition 2.2. Let $G$ be a graph and $x, y \in V(G)$. A 2-separation of $G$ over $\{x, y\}$ is a pair of induced subgraphs $(L, R)$ of $G$ such that:

(1) $E(L) \cup E(R)=E(G)$;

(2) $V(L) \cup V(R)=V(G)$ and $V(L) \cap V(R)=\{x, y\}$;

(3) $V(L)-V(R) \neq \varnothing$ and $V(R)-V(L) \neq \varnothing$.

Note that in Definition 2.2 above, we require $L$ and $R$ to be induced subgraphs, and that $\{x, y\}$ is necessarily a 2 -vertex-cut of $G$.

In this paper, the graphs $K_{4}$ and $K_{2,3}$ have special significance, since they are precisely the excluded minors for $\mathcal{O}$. For simplicity, we define a $K$-graph to be a graph that contains a $K_{4^{-}}$or $K_{2,3}$-subdivision (both of which we call $K$-subdivisions). Equivalently, by Theorem 1.16, $K$-graphs are precisely non-outerplanar graphs. A $K_{2,3}$-subdivision is also known in literature as a $\Theta$-graph.

We observe the following.

Lemma 2.3. If $G$ is 2-connected and contains a $K$-subdivision, then $G=K_{4}$ or $G$ contains a $K_{2,3}$-subdivision. 
Proof. If $G$ contains a $K_{2,3}$-subdivision then we are done. So suppose that $G$ contains a $K_{4}$-subdivision and $G \neq K_{4}$. Then, none of the edges of the $K_{4}$-subdivision are subdivided in $G$, for otherwise $G$ contains a $K_{2,3}$-subdivision. Hence $G$ contains $K_{4}$ as a subgraph, call it $H$. Since $G$ is simple and $G \neq H=K_{4}$, it follows that $G$ has a non-trivial bridge $B$. Since $G$ is 2 -connected, $B$ has at least two feet among the four vertices of $H=K_{4}$. Since $B$ is non-trivial the two feet of $B$ are connected by a path in $B$ of length at least two that is internally disjoint from $H$. Hence, clearly $G$ contains a $K_{2,3}$-subdivision.

\subsection{Starting Point}

Let $\mathcal{S}:=\left\{K_{5}, K_{3,3}\right.$, Oct, $\left.Q, 2 K_{4}, K_{4} \mid K_{2,3}, 2 K_{2,3}\right\}$ be the set of graphs in the figure below.
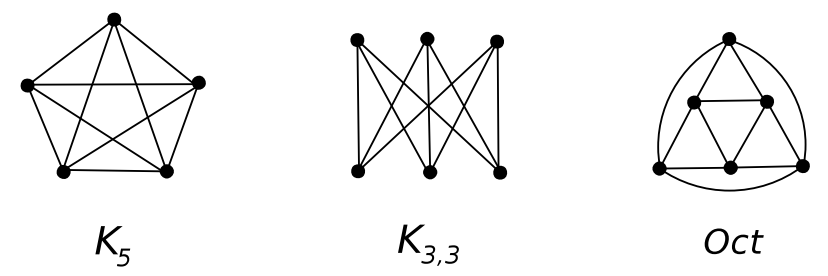

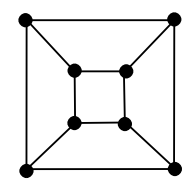

Q

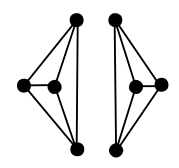

$2 K_{4}$

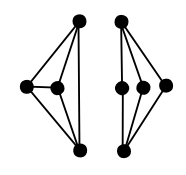

$K_{4} \mid K_{2,3}$

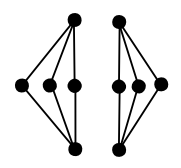

$2 K_{2,3}$

FIGURE 2.1. Starting list of excluded minors for $\mathcal{O}^{*}$

\section{Observation 2.4. $\mathcal{S} \subseteq$ ob $\left(\mathcal{O}^{*}\right)$}

The above observation can easily verified by checking that the graphs themselves do not belong to $\mathcal{O}^{*}$, but all of their single-edge deletions and single-edge contractions contain an apex vertex. We now observe the following.

Observation 2.5. Let $G$ be a graph in $\mathbf{o b}\left(\mathcal{O}^{*}\right)-\mathcal{S}$. Then: 
(1) $G$ is planar

(2) $G$ is 2-connected

(3) $G$ is not 4-connected

Proof. (1) Since $G \ngtr_{m} K_{5}$ and $G \ngtr_{m} K_{3,3}$, it follows that $G$ is planar.

(2) First, suppose that $G$ is disconnected, and let $G$ be a union of two disjoint (not necessarily connected) graphs $G_{1}$ and $G_{2}$. If one of them, say $G_{1}$ is outerplanar, then by the minor-minimality of $G, G_{2}=G-G_{1} \in \mathcal{O}^{*}$, hence $G_{2}$ has a vertex $v$ such that $G_{2}-v \in \mathcal{O}$. Then, $G_{1} \mid\left(G_{2}-v\right) \in \mathcal{O}$, hence $v$ is an apex vertex in $G$, a contradiction. Therefore, both $G_{1}$ and $G_{2}$ are not outerplanar, and so each contains $K_{4}$ or $K_{2,3}$ as a minor. Hence $G$ contains one of $2 K_{4}, K_{4} \mid K_{2,3}, 2 K_{2,3}$ as a minor, a contradiction.

Hence, $G$ is connected. Now suppose that $G$ has a cut-vertex $x$ and let $(L, R)$ be the 1-separation across $x$. By the same argument as above, both $L$ and $R$ are not outerplanar, hence they both contain $K_{4}$ or $K_{2,3}$ as a minor. This implies that both $R-x$ and $L-x$ are outerplanar (for otherwise, $G$ would contain one of $2 K_{4}, K_{4} \mid K_{2,3}, 2 K_{2,3}$ as a minor). Hence $G-x \in \mathcal{O}$, and so $G \in \mathcal{O}^{*}$, a contradiction. Therefore, $G$ is 2-connected.

(3) Suppose that $G$ is 4-connected. Then $\delta(G) \geqslant 4$, and so by Theorem 2.6 below, $G$ contains a $K_{5^{-}}$or $O c t$-minor, a contradiction since $K_{5}, O c t \in \mathcal{S}$

Theorem 2.6. (Halin and Jung [7]) If $\delta(G) \geqslant 4$, then $G$ contains a $K_{5}$ - or Octminor.

It therefore follows by Observation 2.5 that if $G \in \mathbf{o b}\left(\mathcal{O}^{*}\right)-\mathcal{S}$, then the connectivity of $G$ must be either 2 or 3 . The rest of the dissertation is divided into two sections dealing with each case separately. 


\subsection{Key Lemma}

In the remainder of this section we let $G$ be a graph in $\mathbf{o b}\left(\mathcal{O}^{*}\right)-\mathcal{S}$, and we assume that $G$ has connectivity 2 . Note that $G$ cannot contain two disjoint $K$-graphs, for otherwise it would contain one of $2 K_{4}, K_{4} \mid K_{2,3}, 2 K_{2,3}$ as a minor. The following is the key Lemma of this section, which narrows down the structure of $G$.

Lemma 2.7. Let $(L, R)$ be a 2-separation of $G$ over vertices $\{x, y\}$.

(1) If $L \notin \mathcal{O}$ and $R \notin \mathcal{O}$, then one of $L$ and $R$ is one of the five graphs $L_{1}, L_{2}$, $L_{3}, L_{4}, L_{5}$ with prescribed vertices $x$ and $y$, as shown in Figure 2.2.
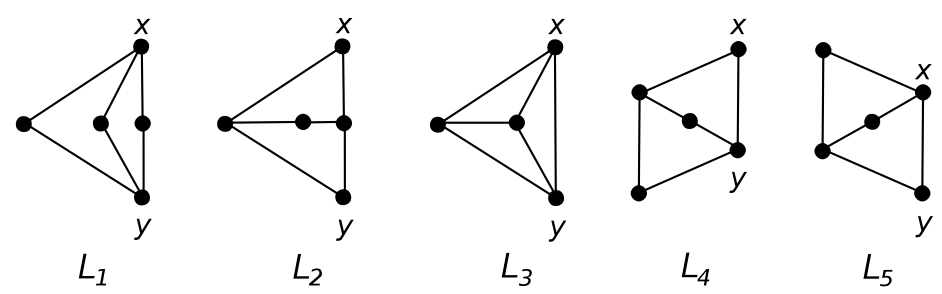

FIGURE 2.2. $K_{4}$ and $K_{2,3}$ 's with prescribed vertices $x$ and $y$

(2) If $L \in \mathcal{O}$, then $x y \notin E(G)$ and $L=P_{2}$ or $C_{4}$, where $P_{2}$ is a path on two edges with endpoints $x$ and $y$, and $C_{4}$ is a cycle on four edges with $x$ and $y$ non-adjacent, as shown in Figure 2.3.

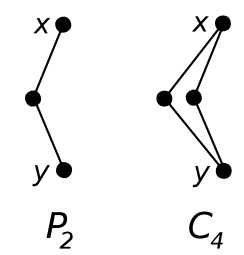

FIGURE 2.3. $P_{2}$ and $C_{4}$

Proof.

(1) Suppose that $L \notin \mathcal{O}$ and $R \notin \mathcal{O}$. Note that $G-\{x, y\} \in \mathcal{O}$, for otherwise $G$ would contain two disjoint $K$-graphs (for instance, $L$ and $R-\{x, y\}$ ). Since $G \notin \mathcal{O}^{*}$, none of its vertices is apex. In particular, since $x$ is not apex in $G$ and $y$ is a cut-vertex in $G-x$, it follows that $L-x$ or $R-x$, say $R-x$, contains a $K$ - 
subdivision, call it $K^{\prime}$, which contains $y$ (since $R-\{x, y\}$ is outerplanar). Similarly, $R-y$ contains a $K$-subdivision (not $L-y$, because such a $K$-subdivision would be disjoint from $K^{\prime}$ ), call it $K^{\prime \prime}$, which contains $x . K^{\prime}$ and $K^{\prime \prime}$ must intersect, otherwise $G$ would contain two disjoint $K$-graphs. Also, $L-x \in \mathcal{O}$ since it is disjoint from $K^{\prime \prime}$, and $L-y \in \mathcal{O}$ since it is disjoint from $K^{\prime}$. Hence, $G$ must have the following structure:

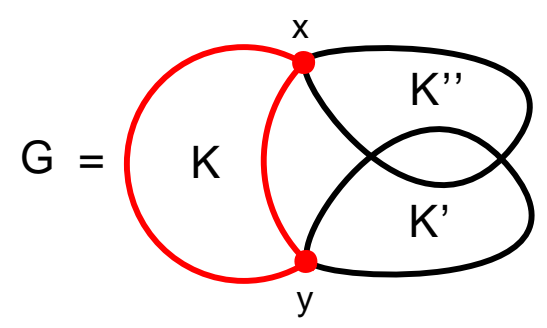

Note that, as long as $L \notin \mathcal{O}$, a graph with the above structure does not belong to $\mathcal{O}^{*}$. This is because none of its vertices is apex: $x$ is not apex, because of $K^{\prime}$; $y$ is not apex, because of $K^{\prime \prime}$; if $v \in L-\{x, y\}$, then $v$ is not apex, because of $K^{\prime}$ (or $K^{\prime \prime}$ ); finally if $v \in R-\{x, y\}$, then $v$ is not apex, because of $L$. Therefore, if $L \notin\left\{K_{4}, K_{2,3}\right\}$, then since $L \notin \mathcal{O}$, it follows that $L$ contains an edge $e$ such that either $L \backslash e \notin \mathcal{O}$, or $L / e \notin \mathcal{O}$ and $e \neq x y$. Hence, either $G \backslash e \notin \mathcal{O}^{*}$ or $G / e \notin \mathcal{O}^{*}$, a contradiction since $G$ is minor-minimal not in $\mathcal{O}^{*}$.

(2) Since $G \notin \mathcal{O}^{*}$, none of its vertices are apex. In particular, since $x$ is not apex, it follows that $R-x$ contains a $K$-subdivision. Similarly, $R-y$ contains a $K$-subdivision. Since $G$ is 2 -connected, it follows that $L$ is connected. We have two cases based on the number of blocks of $L$.

Case 1: $L$ has exactly one block.

Note that $L \neq K_{2}$, for otherwise $(L, R)$ is not a 2-separation. Hence $L$ is 2 connected.

Since $L$ is 2-connected and outerplanar, it follows that $L$ is a cycle $C$ with chords, which has a unique planar embedding such that all the vertices and edges of $C$ 
are incident with the outer face, and all the chords lie in the interior of the disk bounded by $C$. We now show that $L$ has no chords. So, suppose that $L$ does have a chord $e$. Let $s$ be an apex vertex in $G \backslash e$. Then, since $R-x$ and $R-y$ contain $K$-subdivisions, it follows that $s \in V(R-\{x, y\})$. Assume that $(G \backslash e)-s \in \mathcal{O}$ is embedded in the plane so that all of its vertices are incident with the outer face. Then this embedding, restricted to the subgraph $L \backslash e$, is such that all the vertices and edges of $C$ are incident with the outer face. Therefore, by putting the chord $e$ back in, we obtain an embedding of $G-s$ with all of its vertices still incident with the outer face, hence $G-s$ is outerplanar, a contradiction. Hence, we have shown that $L$ has no chords, therefore $L=C$.

Now, suppose that $x$ and $y$ are consecutive vertices of $C$, that is $x y \in E(C)$. Let $s$ be an apex vertex in $G \backslash x y$. Then, again we have that $s \in V(R-\{x, y\})$. Assume that $(G \backslash x y)-s \in \mathcal{O}$ is embedded in the plane so that all of its vertices are incident with the outer face. Since all the vertices of $C-\{x, y\}$ have degree $=2$ in $(G \backslash x y)-s$, it follows that all the edges of $C$ except for $x y$ are incident with the outer face. Therefore, by putting the edge $x y$ back in, we obtain an embedding of $G-s$ with all of its vertices still incident with the outer face, a contradiction.

Therefore, $x$ and $y$ are non-consecutive, which implies that the length of $C$ is at least four. In fact $C=C_{4}$, for suppose that $C=C_{n}$ with $n \geqslant 5$. Then one of the two paths from $x$ to $y$ in $C$ must have length at least three. Let $f$ be an edge on that path with endpoints different from $x$ and $y$. Let $s$ be an apex vertex in $G / f$. Then, again $s \in V(R-\{x, y\})$. Assume that $(G / f)-s \in \mathcal{O}$ is embedded in the plane so that all of its vertices are incident with the outer face. Since all the vertices of $(C / f)-\{x, y\}$ have degree $=2$ in $(G / f)-s$, it follows that all the edges of $C / f$ are incident with the outer face. Therefore, by uncontracting edge $f \in E(C)$, we obtain an embedding of $G-s$ with all of its vertices still incident 
with the outer face, hence $G-s$ is outerplanar, a contradiction. Hence, we have shown that $L=C=C_{4}$.

Therefore, we have shown that if $L$ has only one block, then $L$ is 2 -connected, and in fact $L=C_{4}$ with $x$ and $y$ non-adjacent. Now, we consider the more general case.

Case 2: $L$ has at least two blocks.

Let $B_{x}$ and $B_{y}$ be two distinct blocks containing $x$ and $y$, respectively. Then the block tree of $L$ is, in fact, a path from $B_{x}$ to $B_{y}$, for otherwise $G$ would contain a cut-vertex. Every block on this path is either $K_{2}$ or is 2-connected. If $L$ contains a block $B$ that is 2-connected, then let $s, t \in V(B)$ be the two cut-vertices in $L$ (or in the case of $B_{x}$ and $B_{y}$ the associated pair is given by the corresponding cut-vertex, and $x$ or $y$, respectively). Then since $G$ has a 2 -separation $\left(B, R^{\prime}\right)$ over $\{s, t\}$, it follows by the previous argument that $B=C_{4}$. Therefore, every block of $L$ (which is a path) is either $K_{2}$ or $C_{4}$.

Now suppose that $L$ contains a block $B=C_{4}$, and let $B^{\prime}$ be any other block. Denote by $G / B^{\prime}$ the graph obtained by contracting all the edges of $B^{\prime}$. Again, let $s$ be an apex vertex in $G / B^{\prime}$. Then again $s \in V(R-\{x, y\})$. Assume that $\left(G / B^{\prime}\right)-s \in \mathcal{O}$ is embedded in the plane so that all of its vertices are incident with the outer face. Since two of the non-adjacent vertices of $B$ have degree $=2$ in $\left(G / B^{\prime}\right)-s$ and since all the blocks are either $K_{2}$ or $C_{4}$, it follows that all the edges of $B$ and, in fact, all the edges of $L / B^{\prime}$ are incident with the outer face. Therefore, by uncontracting block $B^{\prime}$, we obtain an embedding of $G-s$ with all of its vertices still incident with the outer face, a contradiction. Hence, we have shown that $L$ does not contain a block $B=C_{4}$, and therefore all the blocks of $L$ are $K_{2}$ 's, or equivalently $L$ is an induced path of length at least two from $x$ to $y$.

Then, in fact, $L=P_{2}$, for suppose that $L=P_{n}$ with $n \geqslant 3$. Let $f$ be an edge 
in $L=P_{n}$ with endpoints different from $x$ and $y$. Let $s$ be an apex vertex in $G / f$. Then, again $s \in V(R-\{x, y\})$. Assume that $(G / f)-s \in \mathcal{O}$ is embedded in the plane so that all of its vertices are incident with the outer face. Since all the vertices of $(L / f)-\{x, y\}$ have degree $=2$ in $(G / f)-s$, it follows that all the edges of $L / f$ are incident with the outer face. Therefore, by uncontracting edge $f$, we obtain an embedding of $G-s$ with all of its vertices still incident with the outer face, a contradiction. Hence, we have shown that $L=P_{2}$. This proves (2).

Finally, we note that 2-separations $(L, R)$ with $L=P_{2}$ or $L=C_{4}$ are indeed realized by graphs in $\mathbf{o b}\left(\mathcal{O}^{*}\right)$ as will be shown later. 


\section{Chapter 3}

\section{Connectivity Two}

\subsection{Roadmap}

Roadmap.

The proof of the connectivity- 2 case will follow the following roadmap. We will split up the case analysis based on the existence of the possible types of 2-separations that can occur in $G$, as indicated by Lemma 2.7. In the following outline of the case structure, all the 2-separations refer to 2-separations $(L, R)$ in $G$ over vertices $\{x, y\}$. Also, $P_{2}$ and $C_{4}$ are as drawn in Figure 2.3, with vertices $\{x, y\}$ as labelled in the Figure.

Case 1: There exists a 2-separation such that both $L \notin \mathcal{O}$ and $R \notin \mathcal{O}$;

Case 2: For each 2-separation, $L=P_{2}$ or $C_{4}$;

Case 2.1: There exists a 2-separation such that $L=C_{4}$;

Case 2.1.1: There exists a 2-separation such that $L=C_{4}$ and $G-\{x, y\} \notin \mathcal{O}$;

Case 2.1.2: There exists a 2-separation such that $L=C_{4}$ and for every such 2-separation $G-\{x, y\} \in \mathcal{O}$;

Case 2.2: For each 2-separation, $L=P_{2}$;

Case 2.2.1: There exists a 2-separation such that $L=P_{2}$ and $G-\{x, y\} \notin \mathcal{O}$;

Case 2.2.2: For each 2-separation, $L=P_{2}$ and $G-\{x, y\} \in \mathcal{O}$.

Note that organizing the case analysis in this way restricts the structure of $G$ more and more as we proceed through the cases.

\subsection{Case 1: Both Sides Non-Outerplanar}

Case 1: There exists a 2-separation such that both $L \notin \mathcal{O}$ and $R \notin \mathcal{O}$. 
By Lemma 2.7, $L \in\left\{L_{1}, L_{2}, L_{3}, L_{4}, L_{5}\right\}$. Note that $R-\{x, y\}$ is outerplanar, for otherwise $G$ contains two disjoint $K$-graphs. Since $G \notin \mathcal{O}^{*}$, none of its vertices is apex. In particular, since $x$ is not apex, $R-x$ contains a $K$-subdivision, which contains $y$ (since $R-\{x, y\}$ is outerplanar). Similarly, $R-y$ contains a $K$-subdivision, which contains $x$. These two $K$-subdivisions must intersect, otherwise $G$ would contain two disjoint $K$-graphs. Hence, $G$ must have the following structure:

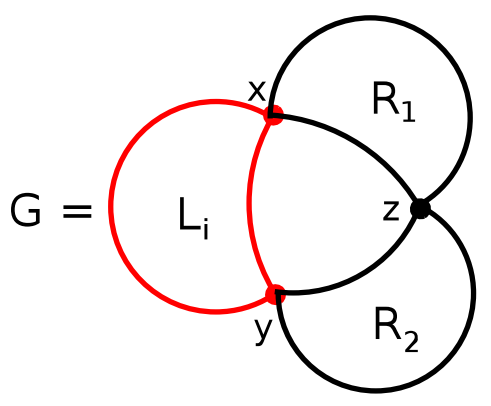

Note that each of the $L_{i}(i=1, \ldots, 5)$ contains $C_{4}$ as a minor (with the vertices $x$ and $y$ preserved). Let $G^{\prime}$ be the graph obtained from $G$ by reducing $L$ (under the minor operation) to $C_{4}$, so that $\left(C_{4}, R\right)$ is a 2-separation of $G^{\prime}$ over $\{x, y\}$. Note that $G^{\prime}$ is a proper minor of $G$, hence by the minor-minimality of $G$, it follows that $G^{\prime} \in \mathcal{O}^{*}$. If there are at least two internally disjoint paths in $R$ from $x$ to $y$, then $G^{\prime}$ has no apex vertex, a contradiction.
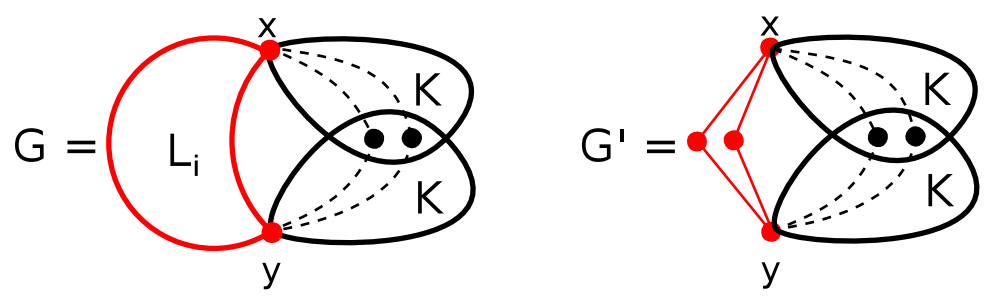

Hence, $R$ has a cut-vertex $z$. Note that $R-z \in \mathcal{O}$, otherwise $R$ contains two disjoint $K$-graphs, a contradiction. 


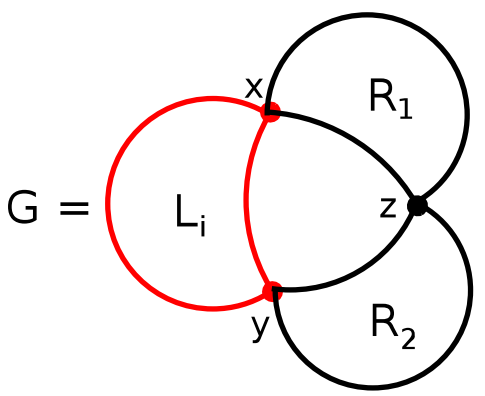

Let $R_{1}$ and $R_{2}$ be the two sides of the 1-separation of $R$ across $z$, such that $x \in R_{1}$ and $y \in R_{2}$. By applying Lemma 2.7 to the 2 -separation in $G$ over $\{x, z\}$, and to the 2-separation in $G$ over $\{y, z\}$, we conclude that both $R_{1}, R_{2} \in\left\{L_{1}, L_{2}, L_{3}, L_{4}, L_{5}\right\}$. Therefore, $G$ is one of the 30 graphs $\left\{T_{1}, T_{2}, \ldots, T_{30}\right\}$ listed in Figure 3.1. It is straightforward to verify that each $T_{i}$ is minor-minimal $\notin \mathcal{O}^{*}$ satisfying the hypothesis of Case 1 . Hence $T_{i} \in \mathbf{o b}\left(\mathcal{O}^{*}\right)$ for $i=1, \ldots, 30$. 

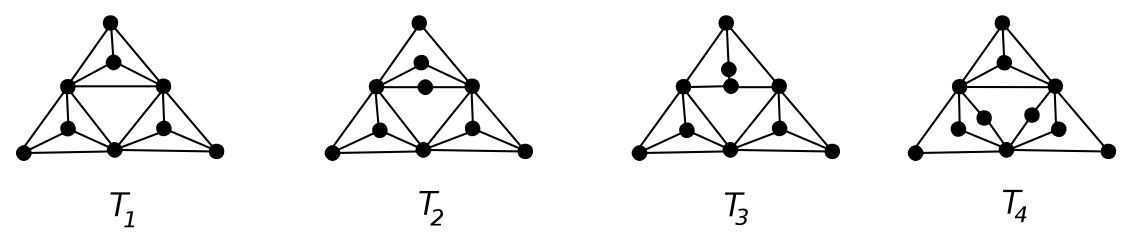

$T_{4}$
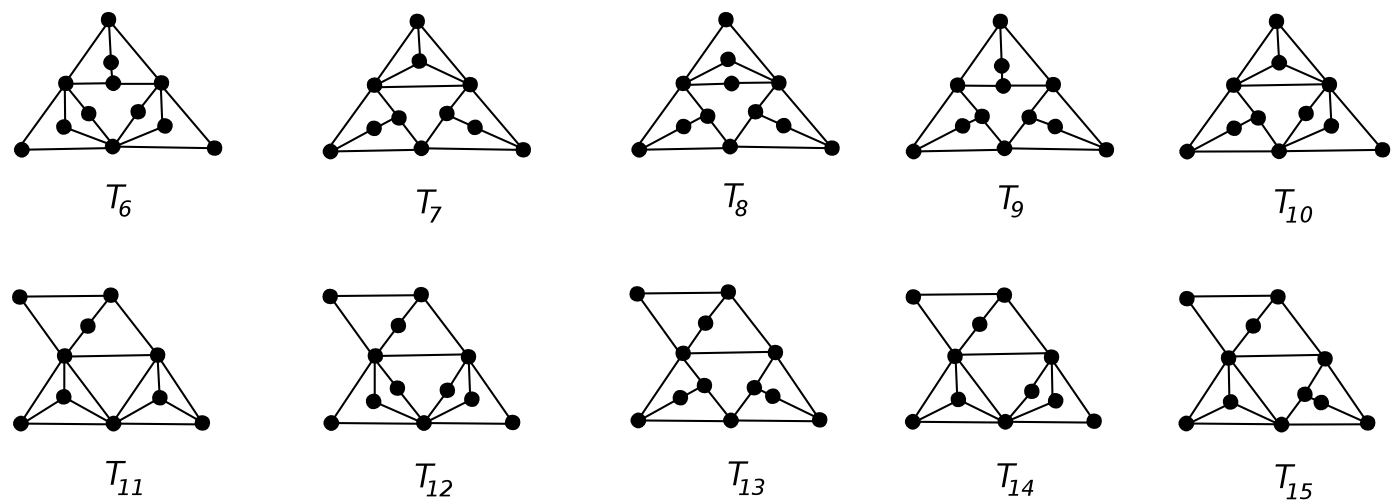

$T_{14}$
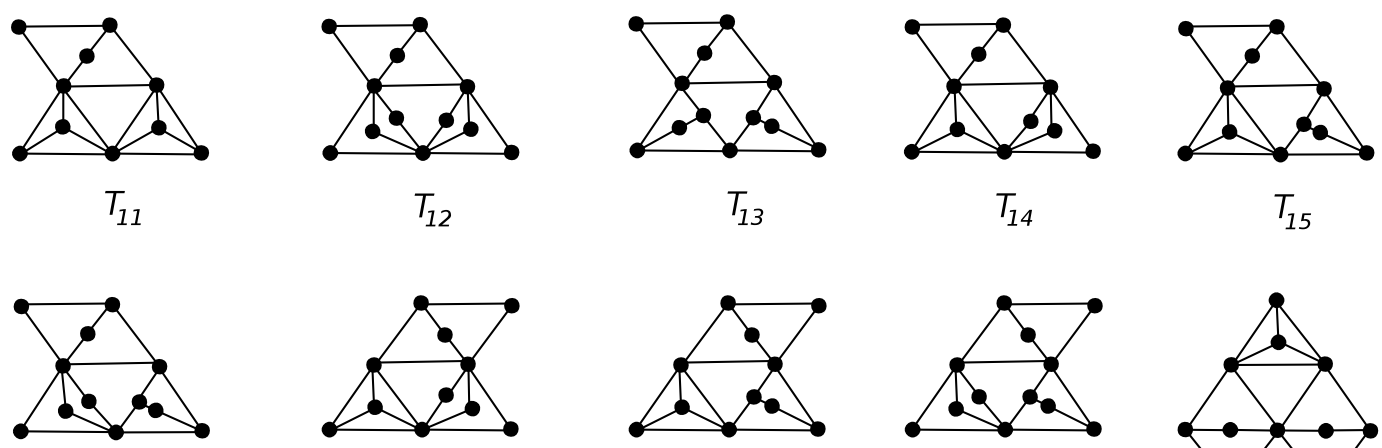

$T_{16}$

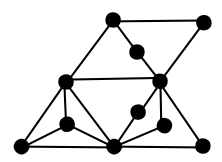

$T_{17}$

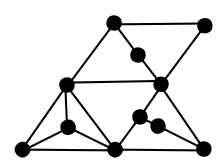

$T_{18}$

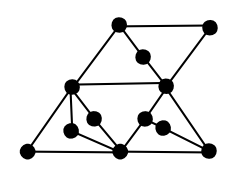

$T_{19}$
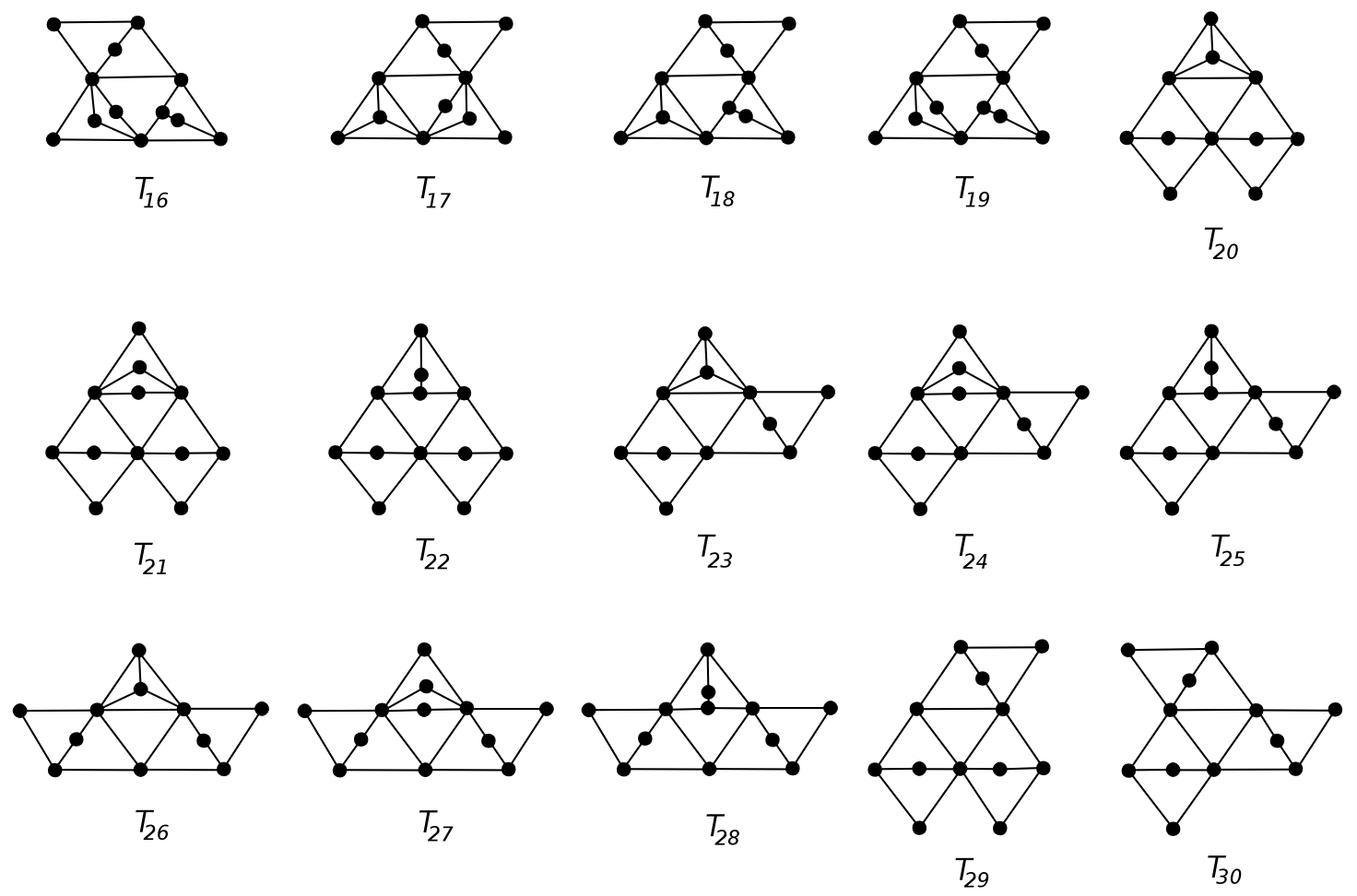

FIGURE 3.1. $\mathcal{T}$ family 


\subsection{Case 2: One Side Outerplanar}

Case 2: For each 2-separation, $L=P_{2}$ or $C_{4}$.

\subsubsection{Case 2.1}

Case 2.1: There exists a 2-separation such that $L=C_{4}$

Case 2.1.1: For each 2-separation, $L=P_{2}$ or $C_{4}$, and there exists a 2-separation such that $L=C_{4}$ with $G-\{x, y\} \notin \mathcal{O}$;

By the hypothesis, it follows that $R-\{x, y\} \notin \mathcal{O}$, hence $R-\{x, y\}$ contains a $K$-subdivision as a subgraph, call it $K^{\prime}$. Note that if $R$ does not have at least two internally disjoint paths from $x$ to $y$, then $R$ has a cut-vertex $z$ separating $x$ and $y$, and hence $G$ has a 2 -separation $\left(L^{\prime}, R^{\prime}\right)$ over $\{x, z\}$ or over $\{y, z\}$ with the property that $R^{\prime} \notin \mathcal{O}$, and either $L^{\prime} \notin \mathcal{O}$ (violating the Case 2 hypothesis) or $L^{\prime} \in \mathcal{O}$ but $L^{\prime}$ different from $P_{2}$ and $C_{4}$ (violating Lemma 2.7), a contradiction. Hence,

(a) $R$ has at least two internally disjoint paths from $x$ to $y$.

Also, note that $R$ does not have a path $P$ from $x$ to $y$ disjoint from $K^{\prime}$, for otherwise $G$ would contain two disjoint $K$-graphs (namely $K^{\prime}$ and the $K_{2,3}$-subdivision formed from the union of $L$ and $P$ ). Therefore $G$ has the following structure:

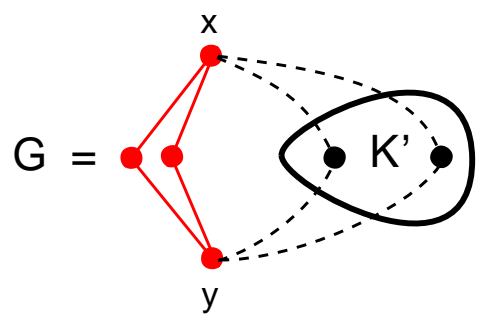

Note that,

(b) A graph with the above structure does not belong to $\mathcal{O}^{*}$.

This is because none of its vertices is apex: if $v \in V(G)-V\left(K^{\prime}\right)$, then $v$ is not apex, because of $K^{\prime}$; and if $v \in V\left(K^{\prime}\right)$, then $R-v$ has a path from $x$ to $y$, which along with $L$ forms a $K_{2,3}$-subdivision in $G-v$, hence $v$ is not apex. 
Fix a planar embedding of $G$. Let $C$ be the outer cycle of $K^{\prime}$. Let $S_{x} \subseteq V(C)$ and $S_{y} \subseteq V(C)$ be the sets of vertices of $C$ from which there is a path to $x$, or respectively to $y$, that doesn't contain other vertices of $C$. It follows, by $(a)$, that $\left|S_{x}\right| \geqslant 2$ and $\left|S_{y}\right| \geqslant 2$, hence $\left|S_{x} \cup S_{y}\right| \geqslant 2$. However, if $\left|S_{x} \cup S_{y}\right|=2$ (see the following figure), then let $\{a, b\}:=S_{x}=S_{y}$, and note that $G$ has a 2-separation $\left(L^{\prime \prime}, R^{\prime \prime}\right)$ over $\{a, b\}$, where $L^{\prime \prime}=K^{\prime} \notin \mathcal{O}$ and $R^{\prime \prime}$ contains a subdivision of $K_{2,4}$, hence $R^{\prime \prime} \notin \mathcal{O}$, contradicting the hypothesis of Case 2 .

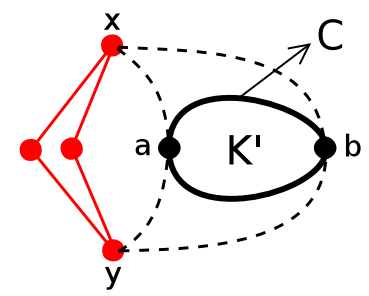

Hence, $\left|S_{x} \cup S_{y}\right| \geqslant 3$. Also, note that, by $(b)$, the paths from $S_{x}$ to $x$ and $S_{y}$ to $y$ are actually simple edges, for otherwise we could perform a contraction along such a path, and by $(b)$, the resulting graph would still be outside of $\mathcal{O}^{*}$, contradicting the minor-minimality of $G$.

Since $K^{\prime}$ is a subdivision of either $K_{4}$ or $K_{2,3}$, it follows that actually $K^{\prime}=K_{4}$ or $K^{\prime}$ is a subdivision of $K_{2,3}$. If $K^{\prime}=K_{4}$, then in view of all the observations above, $G$ is the following graph:

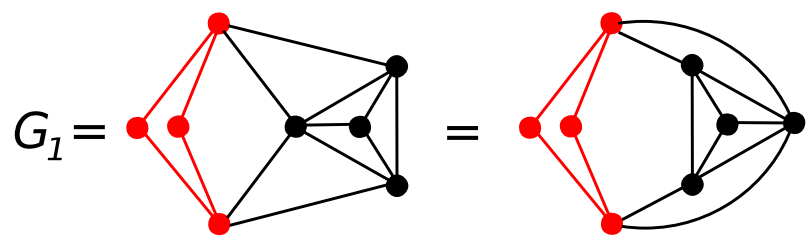

It is easy to verify that the above graph is minor-minimal $\notin \mathcal{O}^{*}$ satisfying the hypothesis of Case 2.1.1. We label it $G_{1}$, and so $G_{1} \in \mathbf{o b}\left(\mathcal{O}^{*}\right)$.

So now, $K^{\prime} \neq K_{4}$, and so $K^{\prime}$ is a subdivision of $K_{2,3}$, or equivalently, $K^{\prime}$ is a $\Theta$-graph. Therefore $K^{\prime}$ consists of the outer cycle $C$ and a path $Q$ of length at least 2 connecting two non-adjacent vertices of $C$. Note that $Q$ has length exactly 
2 , for otherwise we could perform a contraction along $Q$, and by $(b)$, the resulting graph would still be outside of $\mathcal{O}^{*}$, contradicting the minor-minimality of $G$. Let $Q=a, c, b$, so that $a, b \in V(C)$. Then since $K^{\prime}$ is a $\Theta$-graph, we have:

(c) There is at least one vertex in $C(a, b)$ and at least one in $C(b, a)$.

Thus, $G$ has the following structure:

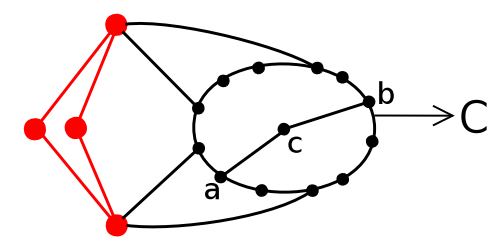

It is straightforward to verify that the following graphs are minor-minimal $\notin \mathcal{O}^{*}$ satisfying the hypothesis of Case 2.1.1 (except the second one, which is minorminimal after contracting $e$; we label this graph $J_{1}$ ). We label them $G_{2}, G_{3}, G_{4}$, $G_{5}$. Hence $J_{1}, G_{i} \in \mathbf{o b}\left(\mathcal{O}^{*}\right)$ for $i=1, \ldots, 5$.
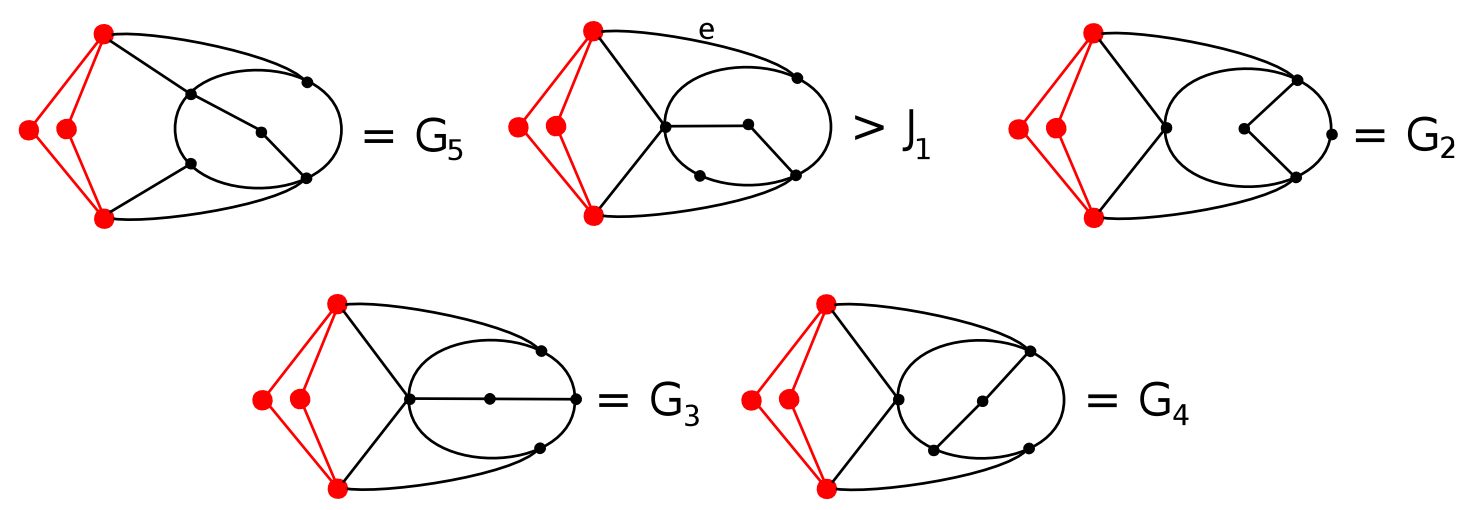

In the remainder of the proof, assume furthermore that $G \notin\left\{J_{1}, G_{1}, G_{2}, G_{3}, G_{4}, G_{5}\right\}$. Let $x_{1}, x_{2} \in S_{x}$ and $y_{1}, y_{2} \in S_{y}$ in the clockwise order $x_{1}, x_{2}, y_{1}, y_{2}$ around $C$. First, assume that all four can be chosen so that they are all distinct. Then, if $a, b \in C\left[x_{1}, x_{2}\right]$ or $a, b \in C\left[y_{1}, y_{2}\right]$, then by $(c), G \geqslant_{m} J_{1}$, a contradiction. If $a, b \in C\left[x_{2}, y_{1}\right]$ or $a, b \in C\left[y_{2}, x_{1}\right]$, then by $(c), G \geqslant_{m} G_{2}$, a contradiction. Finally, if $a$ and $b$ are in distinct segments among $C\left(x_{1}, x_{2}\right), C\left(y_{1}, y_{2}\right), C\left(x_{2}, y_{1}\right), C\left(y_{2}, x_{1}\right)$, or if $\{a, b\}=\left\{x_{1}, y_{1}\right\}$ or if $\{a, b\}=\left\{x_{2}, y_{2}\right\}$, then $G \geqslant_{m} G_{5}$, a contradiction. 

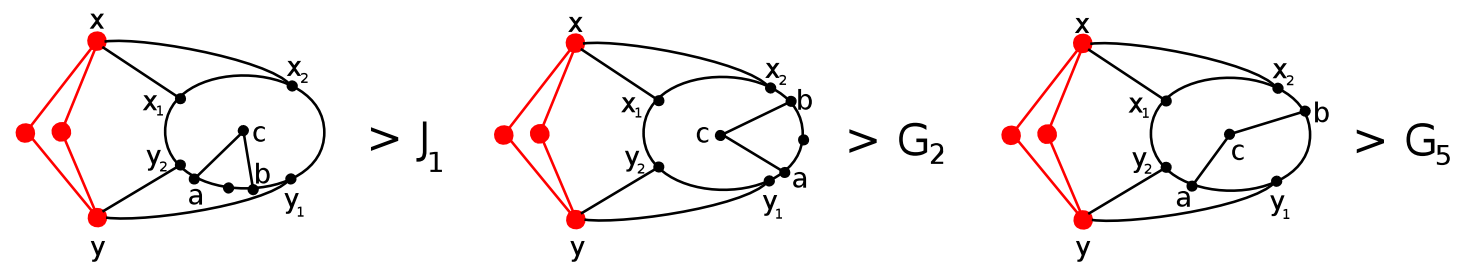

Therefore $x_{1}, x_{2}, y_{1}, y_{2}$ cannot be chosen to be all distinct. Since $\left|S_{x}\right| \geqslant 2$ and $\left|S_{y}\right| \geqslant 2$, and $\left|S_{x} \cup S_{y}\right| \geqslant 3$, it follows that $\left|S_{x} \cup S_{y}\right|=3$. Hence, we let $x_{1}=y_{2}$ and $x_{2} \neq y_{1}$, as in the figure below.

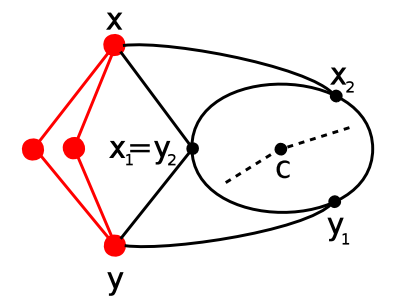

Now, if $a$ is in one of $C\left(x_{1}, x_{2}\right)$ or $C\left(y_{1}, x_{1}\right)$, say $C\left(y_{1}, x_{1}\right)$, then: if $b \in C\left[y_{1}, x_{1}\right]$, then by $(c), G \geqslant_{m} J_{1}$, a contradiction; if $b \in C\left(x_{1}, x_{2}\right]$, then $G \geqslant_{m} G_{4}$; finally, if $b \in C\left(x_{2}, y_{1}\right)$, then $G \geqslant_{m} G_{3}$. Hence, we have shown that neither $a$ nor $b$ can be in $C\left(x_{1}, x_{2}\right) \cup C\left(y_{1}, x_{1}\right)$. If $a=x_{1}$, then if $b=x_{2}$ or $y_{1}$, then by $(c), G \geqslant_{m} J_{1}$, a contradiction; and if $b \in C\left(x_{2}, y_{1}\right)$, then $G \geqslant_{m} G_{3}$, a contradiction. So finally, both $a$ and $b$ must be in $C\left[x_{2}, y_{1}\right]$. But, then it follows by $(c)$ that $G \geqslant_{m} G_{2}$, a contradiction. This concludes the proof of Case 2.1.1.

Finally, Figure 3.2 shows slightly different embeddings of the $G_{i}$ 's from the ones above. 

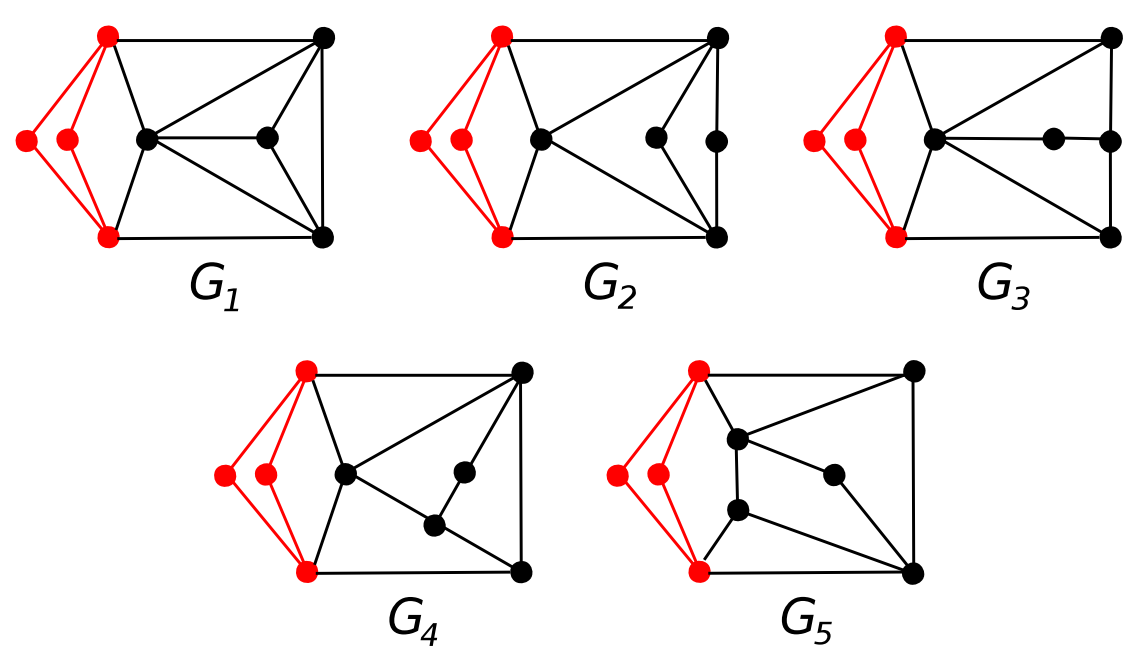

FIGURE 3.2. $\mathcal{G}$ family

Case 2.1.2: For each 2-separation, $L=P_{2}$ or $C_{4}$, and there exists a 2-separation such that $L=C_{4}$, and for every such 2-separation $G-\{x, y\} \in \mathcal{O}$.

It is straightforward to verify that the graphs in Figure 3.3 are minor-minimal $\notin \mathcal{O}^{*}$ satisfying the hypothesis of Case 2.1.2. We label them $J_{1}, J_{2}, J_{3}, J_{4}, J_{5}$. Hence $J_{i} \in \mathbf{o b}\left(\mathcal{O}^{*}\right)$ for $i=1, \ldots, 5$.
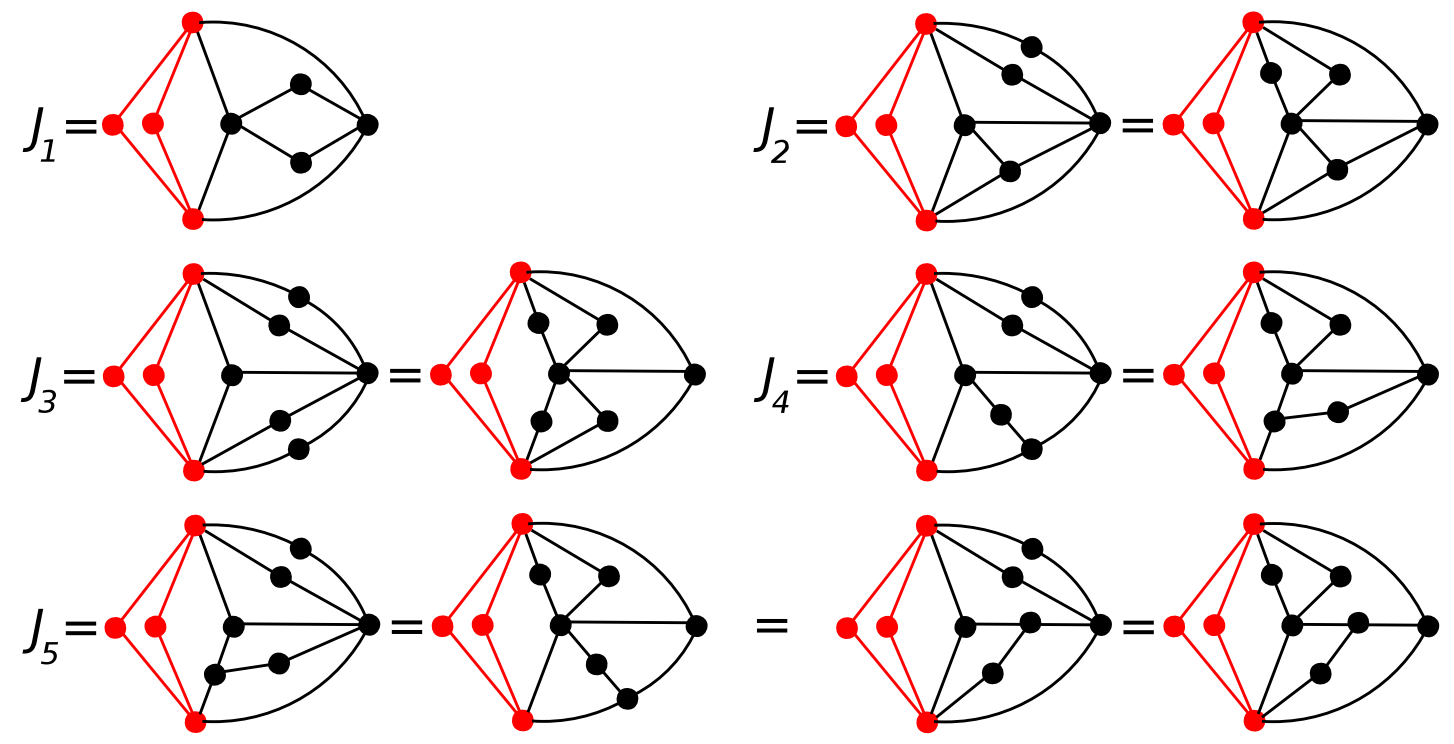

FIGURE 3.3. $\mathcal{J}$ family 
In the remainder of the proof, assume furthermore that $G \notin\left\{J_{1}, J_{2}, J_{3}, J_{4}, J_{5}, Q_{2}\right\}$, where $Q_{2} \in \mathbf{o b}\left(\mathcal{O}^{*}\right)$ as will be shown in Case 2.2.2. Since $R-\{x, y\} \in \mathcal{O}$, it follows by the same arguments as in Case 1, that $G$ must have the following structure:

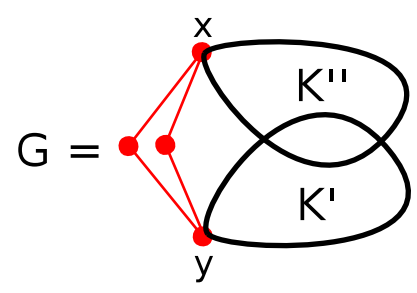

where $K^{\prime}$ is a $K$-subdivision contained in $R-x$ containing $y$ (so that $K^{\prime}-y \in \mathcal{O}$ ), and $K^{\prime \prime}$ is a $K$-subdivision contained in $R-y$ containing $x$ (so that $K^{\prime \prime}-x \in \mathcal{O}$ ). Note that,

(a) $R$ does not have a path $P$ from $x$ to $y$ that is internally disjoint from $K^{\prime} \cup K^{\prime \prime}$. For otherwise, $G$ would have a 2-separation $\left(L^{\prime}, R^{\prime}\right)$ over $\{x, y\}$, with $L^{\prime}=L \cup P \notin$ $\mathcal{O}$ and $R^{\prime}=R \notin \mathcal{O}$, contradicting the hypothesis of Case 2 .

Also, note that if $R$ does not have at least two internally disjoint paths from $x$ to $y$, then $R$ has a cut-vertex $z$. Note that $z$ lies at the intersection of $K^{\prime}$ and $K^{\prime \prime}$ (for otherwise $K^{\prime}$ and $K^{\prime \prime}$ would be disjoint, or $R-\{x, y\}$ would not be outerplanar). But, $R-z \in \mathcal{O}$ (for otherwise $K^{\prime}$ and $K^{\prime \prime}$ would be disjoint), therefore $G-z \in \mathcal{O}$, a contradiction. Hence, $R$ has at least two internally disjoint paths from $x$ to $y$.
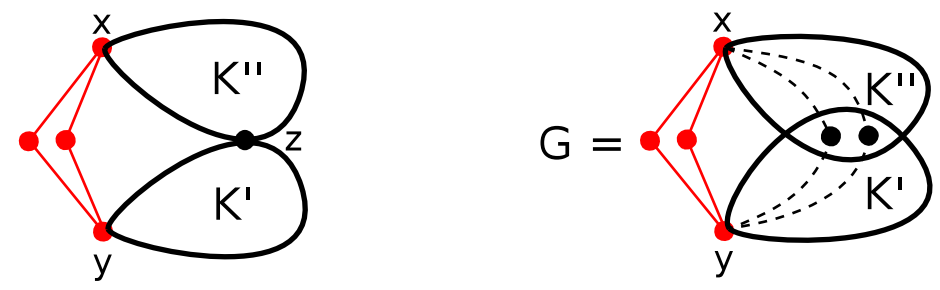

Note that,

(b) a graph with the above structure does not belong to $\mathcal{O}^{*}$.

This is because none of its vertices is apex: if $v \in V(G)-V\left(K^{\prime}\right)$, then $v$ is not apex, because of $K^{\prime}$; if $v \in V(G)-V\left(K^{\prime \prime}\right)$, then $v$ is not apex, because of $K^{\prime \prime}$; and 
if $v \in V\left(K^{\prime}\right) \cap V\left(K^{\prime \prime}\right)$, then $R-v$ has a path from $x$ to $y$, which along with $L$ forms a $K_{2,3}$-subdivision in $G-v$, hence $v$ is not apex.

Fix a planar embedding of $G$. Since $R$ does not have a cut-vertex, it is 2 connected. Let $C$ be the outer cycle of $R$, so that the rest of $R$ is embedded in the closed disk bounded by $C$. Let $P_{1}$ and $P_{2}$ be the two internally disjoint paths from $x$ to $y$ whose union is $C$. Note that neither $P_{1}$ nor $P_{2}$ is a simple edge, since $x y \notin E(G)$. Note that,

(c) There must be a path $P_{3}$ between $\operatorname{int}\left(P_{1}\right)$ and $\operatorname{int}\left(P_{2}\right)$ such that $V\left(P_{3}\right) \cap V(C)=$ $\{a, b\}$, where $a \in \operatorname{int}\left(P_{1}\right)$ and $b \in \operatorname{int}\left(P_{2}\right)$ are the endpoints of $P_{3}$.

For otherwise, one of $\operatorname{int}\left(P_{1}\right)$ or $\operatorname{int}\left(P_{2}\right)$ would be vertex-disjoint from $K^{\prime} \cup K^{\prime \prime}$, contradicting $(a)$.

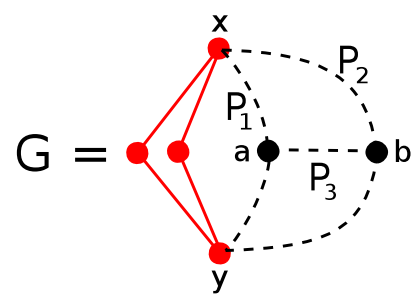

Let $\mathcal{P}$ be the set of paths with property $(c)$. By $(c)$, it follows that $|\mathcal{P}| \geqslant 1$. The remainder of the analysis for this case is based on the length $l(\mathcal{P})$ of the longest path in $\mathcal{P}$.

Case 2.1.2a: $l(\mathcal{P})=1$.

By hypothesis, all the paths in $\mathcal{P}$ are simple edges. Let $a_{1}, a_{2}, \ldots, a_{s} \in \operatorname{int}\left(P_{1}\right)$ be the left endpoints of the paths in $\mathcal{P}$ in the order of vertices in $P_{1}$ from $x$ to $y$, and similarly let $b_{1}, b_{2}, \ldots, b_{t} \in \operatorname{int}\left(P_{2}\right)$ be the right endpoints of the paths in $\mathcal{P}$ in the order of vertices in $P_{2}$ from $x$ to $y$. Note that, for $i=1, \ldots, s-1$ (and for $j=1, \ldots t-1)$, if $a_{i} a_{i+1}\left(\right.$ or $\left.b_{j} b_{j+1}\right)$ is not a simple edge, then $G$ has a 2 -separation $\left(L^{\prime}, R^{\prime}\right)$ over $\left\{a_{i}, a_{i+1}\right\}$ (or over $\left.\left\{b_{j}, b_{j+1}\right\}\right)$. By the Case 2 hypothesis, $L^{\prime}=P_{2}$ or 
$C_{4}$. However, by the hypothesis of Case 2.1.2, $L^{\prime} \neq C_{4}$, because $G-\left\{a_{i}, a_{i+1}\right\}$ (and $\left.G-\left\{b_{j}, b_{j+1}\right\}\right)$ contains a $K_{2,3}$-subdivision. Hence,

(d) for $i=1, \ldots, s-1$ and for $j=1, \ldots t-1, a_{i} a_{i+1}$ and $b_{j} b_{j+1}$ are either simple edges or edges subdivided once.

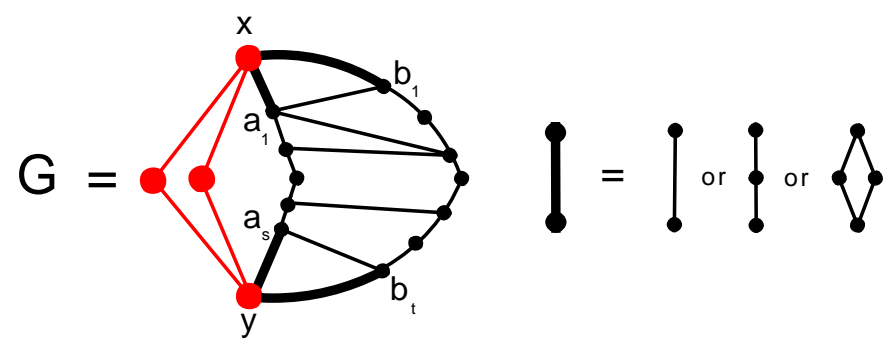

Similarly, if $x a_{1}, x b_{1}, y a_{s}$, or $y b_{t}$ is not a simple edge, then $G$ has a 2-separation $\left(L^{\prime}, R^{\prime}\right)$ over the corresponding 2 -vertex set, and by the Case 2 hypothesis, $L^{\prime}=P_{2}$ or $C_{4}$. If $L^{\prime}\left(x, a_{1}\right)=C_{4}$ and $L^{\prime}\left(y, a_{s}\right)=C_{4}\left(\right.$ or $L^{\prime}\left(x, b_{1}\right)=C_{4}$ and $\left.L^{\prime}\left(y, b_{t}\right)=C_{4}\right)$, then $G \geqslant_{m} J_{3}$, a contradiction (see figure below). Similarly, $L^{\prime}\left(x, a_{1}\right)=C_{4}$ and $L^{\prime}\left(y, b_{t}\right)=C_{4}$ (or $L^{\prime}\left(x, b_{1}\right)=C_{4}$ and $\left.L^{\prime}\left(y, a_{s}\right)=C_{4}\right)$, then $G \geqslant_{m} J_{1}$, a contradiction (see figure below).

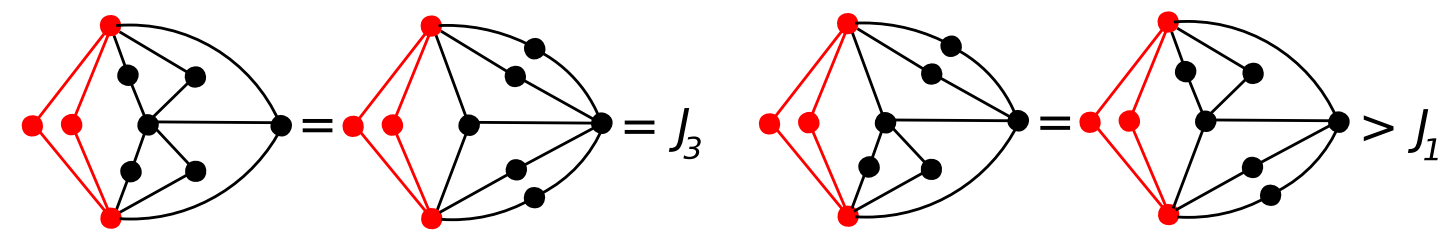

Therefore, for one of the sides, say the $x$-side, we must have that $x a_{1}$ and $x b_{1}$ are either simple edges, or edges subdivided once. Therefore, it follows by $(d)$ that the vertex $y$ is apex, a contradiction since $G \notin \mathcal{O}^{*}$.

Case 2.1.2b: $l(\mathcal{P}) \geqslant 2$.

Let $P=p_{0} p_{1} \ldots p_{n}$ be a path in $\mathcal{P}$ of length $n:=l(\mathcal{P})$, with $p_{0} \in \operatorname{int}\left(P_{1}\right)$ and $p_{n} \in \operatorname{int}\left(P_{2}\right)$. Since $G \ngtr_{m} J_{1}$, it follows that:

(e) for $i=0,1, \ldots, n-2$, there is no path of length $\geqslant 2$ from $p_{i}$ to $\operatorname{int}\left(P_{2}\right)$ that is internally disjoint from $P \cup C$.

Note that, by choice of $P$, the same holds true for $i=n-1$. Similarly: 
$(f)$ for $i=2,3, \ldots, n$, there is no path of length $\geqslant 2$ from $p_{i}$ to $\operatorname{int}\left(P_{1}\right)$ that is internally disjoint from $P \cup C$.

And, by choice of $P$, the above also holds true for $i=1$. Therefore, equivalently: $\left(e^{\prime}\right)$ for $i=0,1, \ldots, n-1$, all the paths from $p_{i}$ to $\operatorname{int}\left(P_{2}\right)$ that are internally disjoint from $P \cup C$ are simple edges.

$\left(f^{\prime}\right)$ for $i=1,2, \ldots, n$, all the paths from $p_{i}$ to $\operatorname{int}\left(P_{1}\right)$ that are internally disjoint from $P \cup C$ are simple edges.

Let $P_{11}$ and $P_{12}$ be the subpaths of $P_{1}$ from $x$ to $p_{0}$, and from $p_{0}$ to $y$, respectively. Similarly, Let $P_{21}$ and $P_{22}$ be the subpaths of $P_{2}$ from $x$ to $p_{n}$, and from $p_{n}$ to $y$, respectively. Let $C_{x}$ be the cycle formed from the union of the paths $P, P_{11}$ and $P_{21}$, and let $C_{y}$ be the cycle formed from the union of the paths $P, P_{12}$ and $P_{22}$.

Again, since $G \ngtr_{m} J_{1}$, it follows that:

(g) all the paths in $\mathcal{P}$ that are internally disjoint from $P$ are simple edges.

It follows by $\left(e^{\prime}\right)$ and $\left(f^{\prime}\right)$, that $G$ does not have a bridge with one foot in $\operatorname{int}(P)$ and another in $\operatorname{int}\left(P_{1}\right) \cup \operatorname{int}\left(P_{2}\right)$. Also, if $G$ has a bridge with two feet in $P$, then if the feet are consecutive vertices of $P$, then this violates the choice of $P$; and if they are non-consecutive, then $G \geqslant_{m} J_{1}$, a contradiction. Therefore:

(h) the only bridges of $G$ that attach to $\operatorname{int}(P)$ have one foot in $\operatorname{int}(P)$ and the other at $x$ or $y$.

Let $B$ be a bridge that attaches to $\operatorname{int}(P)$. Then, it follows by $(h)$ that $B$ has one foot, call it $p$, in $\operatorname{int}(P)$ and the other at $x$ or $y$, say $x$. Then $G$ has a 2 -separation $\left(L^{\prime}, R^{\prime}\right)$ over $\{x, p\}$, and it follows by the Case 2 hypothesis that $L^{\prime}=P_{2}$ or $C_{4}$. Hence, $B-\{x, p\}$ is a single vertex, or a pair of non-adjacent vertices. We call such a bridge a $P_{2}$-bridge, or a $C_{4}$-bridge over $\{x, p\}$, respectively. Thus we have shown: $\left(h^{\prime}\right)$ If $B$ is a bridge with one foot $p \in \operatorname{int}(P)$ and the other at $x$ (or $\left.y\right)$, then $B$ is a $P_{2^{-}}$or $C_{4}$-bridge over $\{x, p\}$ (over $\{y, p\}$ respectively). 
Let $F_{0}$ be the set of edges with one endpoint in $\operatorname{int}\left(P_{1}\right)-\left\{p_{0}\right\}$ and the other in $\operatorname{int}\left(P_{2}\right)-\left\{p_{n}\right\}$, and let $F_{1}$ be the set of edges whose both endpoints are nonconsecutive vertices of $P$. Let $F_{2}$ be the set of edges with one endpoint in $\left\{p_{0}, p_{1}, \ldots, p_{n-2}\right\}$ and the other in $\operatorname{int}\left(P_{2}\right)-\left\{p_{n}\right\}$, and let $F_{3}$ be the set of edges with one endpoint in $\left\{p_{2}, p_{3}, \ldots, p_{n}\right\}$ and the other in $\operatorname{int}\left(P_{1}\right)-\left\{p_{0}\right\}$. Note that $F_{0}, F_{1}, F_{2}$, and $F_{3}$ are pairwise disjoint. Let $F:=F_{0} \cup F_{1} \cup F_{2} \cup F_{3}$ if $n \geq 3$. For shorthand, we will say that an edge or a vertex is embedded in the top or in the bottom, if it is embedded in the closed disk bounded by $C_{x}$ or in the closed disk bounded by $C_{x}$, respectively. We prove the following:

(i) If $F \neq \varnothing$, then all edges of $F$ can be embedded on one side: top or bottom.

First, assume that this is impossible for two edges $e$ and $f$ of $F_{1}$. If the endpoints of $e=p_{i_{0}} p_{i_{1}}$ and $f=p_{i_{2}} p_{i_{3}}$ overlap, in the sense that $i_{0}<i_{2}<i_{1}<i_{3}$, then $G \geqslant_{m} J_{1}$, a contradiction (see figure below).

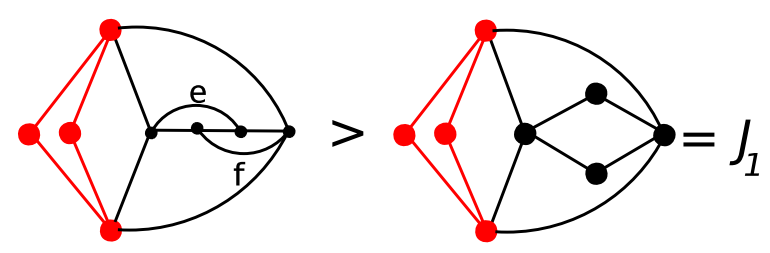

If the endpoints of $e$ and $f$ do not overlap (in the sense that $i_{0}<i_{1}<i_{2}<i_{3}$ ) and, without loss of generality, $e$ is in the top and $f$ is in the bottom, then since $G$ does not have a 2 -separation over $\left\{p_{i_{0}}, p_{i_{1}}\right\}$ (by the Case 2 hypothesis), and since the vertices $p_{i_{0}}, p_{i_{1}}$ are non-consecutive in $P$, there is a path from a vertex in $P\left(p_{i_{0}}, p_{i_{1}}\right)$ to $P_{12}$ (note that if the path is to a vertex in $\operatorname{int}\left(P_{2}\right)$, then $G \geqslant_{m} J_{1}$ as in the overlapping case; and similarly if the path is to a vertex $p_{i_{4}} \in P$ for some $i_{4}<i_{0}$ or $i_{4}>i_{1}$ ). Similarly, since $G$ does not have a 2-separation over $\left\{p_{i_{2}}, p_{i_{3}}\right\}$, there is a path from a vertex in $P\left(p_{i_{2}}, p_{i_{3}}\right)$ to $P_{21}$. Therefore $G \geqslant_{m} Q_{2}$, a contradiction (see figure below). 


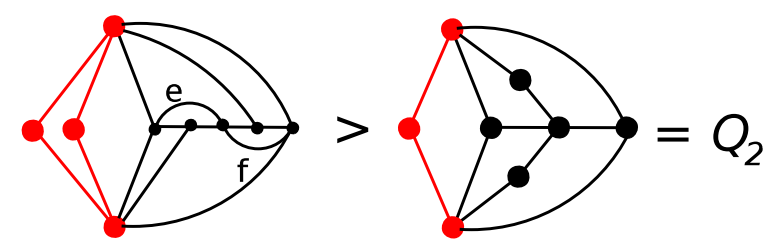

Second, assume that $(i)$ is impossible due to two edges $e$ and $f$ of $F_{2}$ (the proof for $F_{3}$ is similar). Hence, both $e$ and $f$ have one endpoint in $\left\{p_{0}, p_{1}, \ldots, p_{n-2}\right\}$, however $e$ has the other endpoint in $\operatorname{int}\left(P_{21}\right)$ and $f$ in $\operatorname{int}\left(P_{22}\right)$. Then, $G-\{x, y\}$ contains a $K_{2,3}$-subdivision, contradicting the hypothesis of Case 2.1.2.

Third, assume that $(i)$ is impossible due to an edge $e \in F_{2}$, embedded, say, in the bottom, and an edge $f \in F_{3}$ embedded in the top. Then $G$ contains the following minor, which contains a $Q_{2}$-minor, a contradiction (see figure below).

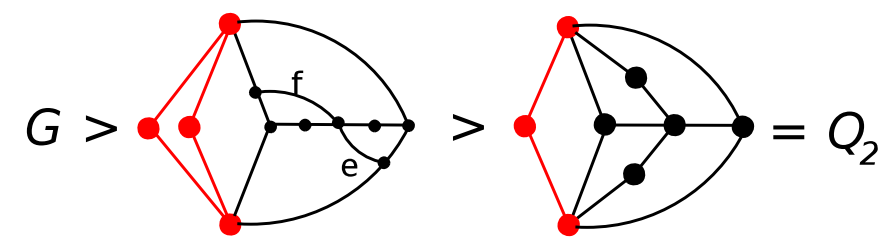

Fourth, assume that $(i)$ is impossible due to an edge $e \in F_{1}$, embedded, say, in the bottom, and an edge $f \in F_{2}$ (the proof for $f \in F_{3}$ is similar) embedded in the top. Let $p_{i_{0}} q:=f$ with $i_{0} \in\{0,1, \ldots, n-2\}$ and $q \in \operatorname{int}\left(P_{21}\right)$, and let $p_{i_{1}} p_{i_{2}}:=e$ with $i_{1}<i_{2}$. If $i_{1} \geqslant i_{0}$, then $G-\{x, y\}$ contains a $K_{4}$-subdivision, a contradiction. Hence, $i_{1}<i_{0}$. If $i_{2}=n$, then since $i_{0} \in\{0,1, \ldots, n-2\}$, it follows that $G-\{x, y\}$ contains a $K_{2,3}$-subdivision, a contradiction. If $i_{2} \in\left(i_{0}, n-1\right]$, then $G \geqslant_{m} J_{1}$ (as in the overlapping case), a contradiction. Therefore, $i_{2} \leqslant i_{0}$ and since $G$ does not have a 2 -separation over $\left\{p_{i_{1}}, p_{i_{2}}\right\}$ (by the Case 2 hypothesis), there is a path from a vertex in $P\left(p_{i_{1}}, p_{i_{2}}\right)$ to $P_{12}-\left\{p_{0}\right\}$, and thus $G$ contains the following minor, which contains a $Q_{2}$-minor, a contradiction (see figure below).

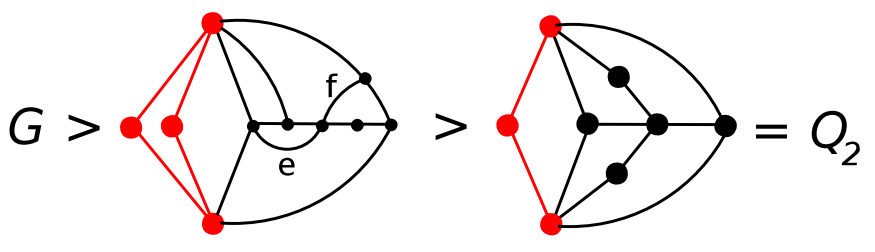


Finally, assume that $(i)$ is impossible due to an edge $e \in F_{0}$, embedded, say, in the top, and an edge $f \in F_{0} \cup F_{1} \cup F_{2} \cup F_{3}$ embedded in the bottom (the case $f \in F_{0}$ is illustrated below). Then, it can easily be checked that $G-\{x, y\}$ contains a $K_{4^{-}}$or $K_{2,3^{-}}$subdivision, a contradiction. This proves $(i)$.

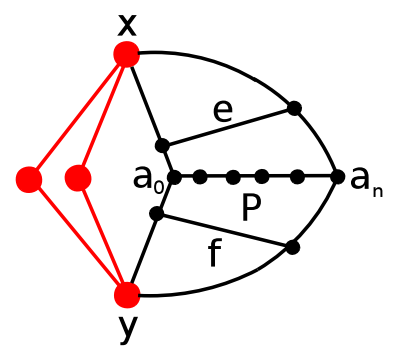

As in Case 2.1.2a, let $a_{1}, a_{2}, \ldots, a_{s} \in \operatorname{int}\left(P_{1}\right)$ be the left endpoints of the paths in $\mathcal{P}$ in the order of vertices on $P_{1}$ from $x$ to $y$, and similarly let $b_{1}, b_{2}, \ldots, b_{t} \in \operatorname{int}\left(P_{2}\right)$ be the right endpoints of the paths in $\mathcal{P}$ in the order of vertices on $P_{2}$ from $x$ to $y$. Similarly to $(d)$ in Case 2.1.2a, we have that:

(j) for $i=1, \ldots, s-1$ and for $j=1, \ldots t-1, a_{i} a_{i+1}$ and $b_{j} b_{j+1}$ are either simple edges or edges subdivided once.

Similarly, if $x a_{1}, x b_{1}, y a_{s}$, or $y b_{t}$ is not a simple edge, then $G$ has a 2-separation $\left(L^{\prime}, R^{\prime}\right)$ over the corresponding 2 -vertex set, and by the Case 2 hypothesis, $L^{\prime}=P_{2}$ or $C_{4}$. Thus:

(k) If $x a_{1}, x b_{1}, y a_{s}$, or $y b_{t}$ is not a simple edge, then $L^{\prime}\left(x, a_{1}\right), L^{\prime}\left(x, b_{1}\right), L^{\prime}\left(y, a_{s}\right)$, $L^{\prime}\left(y, b_{t}\right) \in\left\{P_{2}, C_{4}\right\}$, respectively (equivalently, $G$ has a $P_{2^{-}}$or $C_{4^{-}}$-bridge over $\left\{x, a_{1}\right\},\left\{x, b_{1}\right\},\left\{y, a_{s}\right\}$, or $\left\{y, b_{t}\right\}$, respectively)

We will first consider the case that $F \neq \varnothing$. Then, it follows from $(i)$ that all the edges of $F$ can be embedded, say, in the bottom (hence there are no edges of $F$ embedded in the top). We will show that since $G$ does not contain $J_{i}$-minor for 
$i=1, \ldots, 5$, the vertex $x$ will be apex in $G$, obtaining a contradiction. To do this, we first prove the following Claim.

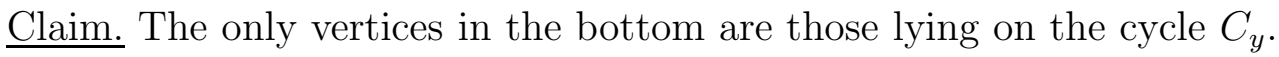

We prove the above claim by showing that there are no bridges in the interior of the disk bounded by $C_{y}$. So assume that there is such a bridge $B$. First, if $B$ has a foot in $\operatorname{int}(P)$, then by $(h)$, it follows that the other foot of $B$ is $y$. Since $F \neq \varnothing$, it contains an edge $e \in F_{i}$ for some $i=0,1,2,3$. Actually, $e \notin F_{0}$, for otherwise $e$ would cross $B$, a contradiction. If $e \in F_{1}$, then $G$ contains the following minor, which contains a $Q_{2}$-minor, contradiction (see figure below).

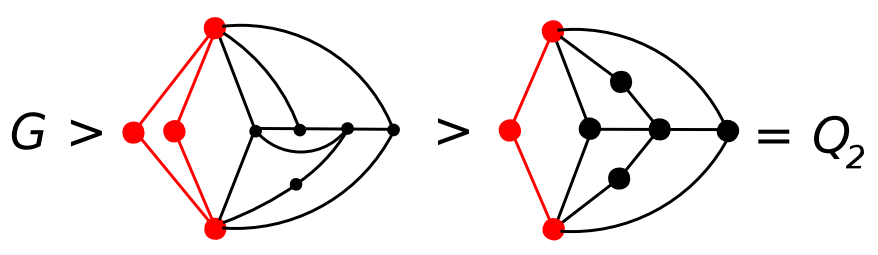

And if $e \in F_{2}$ (the proof for $F_{3}$ is similar), then $G$ contains the following minor, which again contains a $Q_{2}$-minor, contradiction (see figure below).

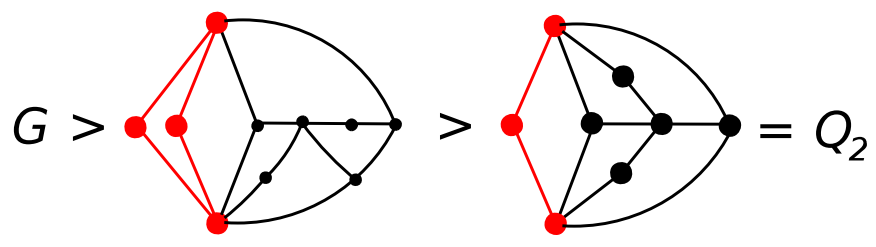

Therefore $B$ has its feet in $P_{12} \cup P_{22}$, but it cannot have a foot in $P_{12}$ and another in $P_{22}$, because this would contradict either $\left(e^{\prime}\right),\left(f^{\prime}\right)$, or $(g)$. Hence, $B$ has all of its feet in $P_{12}$ or all in $P_{22}$; by symmetry, we may assume that in $P_{12}$. Let $p$ and $q$ be the first and last feet of $B$ in the order of vertices on $P_{12}$. Then $G$ has a 2-separation $\left(L^{\prime}, R^{\prime}\right)$ over $\{p, q\}$, and by the Case 2 hypothesis, $L^{\prime}=P_{2}$ or $C_{4}$, so that $B$ is a $P_{2^{-}}$or $C_{4}$-bridge over $\{p, q\}$. Since $F \neq \varnothing$, it follows that $B \neq C_{4}$ for otherwise $G$ would contain a $J_{2^{-}}, J_{4^{-}}$, or $J_{5^{-}}$minor (see figure below). 

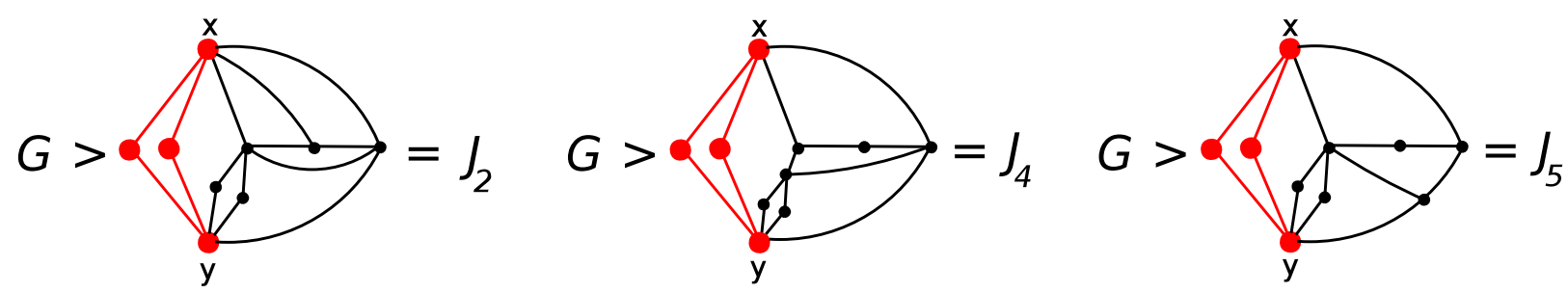

Hence, $B=P_{2}$, and so $B$ is a subgraph of $P_{12}$. This proves the Claim.

Hence, it follows by the Claim, that $L^{\prime}\left(y, a_{s}\right) \neq C_{4}$ and $L^{\prime}\left(y, b_{t}\right) \neq C_{4}$. Hence, $y a_{s}$ and $y b_{t}$ are either simple edges, or edges subdivided once. However, $L^{\prime}\left(x, a_{1}\right)$ and $L^{\prime}\left(x, b_{1}\right)$ could be either $P_{2}$ or $C_{4}$, or $x a_{1}$ and $x b_{1}$ could be simple edges.

By the fact that there are no edges of $F$ in the top, and from $(h),\left(h^{\prime}\right),(j)$, and $(k)$, it follows that the only possible edges in the top are:

- edges from $p_{1}$ to $P_{11}$;

- edges from $p_{n-1}$ to $P_{21}$;

- edges from $\operatorname{int}(P)$ to $x$;

- edges that are part of the $P_{2^{-}}$or $C_{4^{-}}$-bridges from $\operatorname{int}(P)$ to $x$;

- edges that are part of the $P_{2^{-}}$or $C_{4}$-bridges from $a_{1}$ or $b_{1}$ to $x$;

- edges of the cycle $C_{x}$;

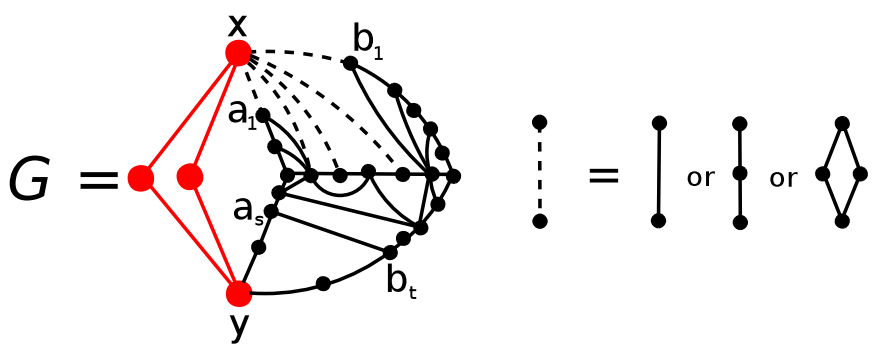

Hence, the only possible vertices lying in the interior of the disk bounded by $C_{x}$ are those from the $P_{2^{-}}$or $C_{4}$-bridges from $\operatorname{int}(P) \cup\left\{a_{1}, b_{1}\right\}$ to $x$. Hence, from this and the Claim it follows that $G-x$ is outerplanar (i.e. $x$ is an apex vertex of $G$ ), a contradiction. 
Finally, we consider the case that $F=\varnothing$. Again, by the fact that there are no edges of $F$ in the top and none in the bottom, and from $(h),\left(h^{\prime}\right),(j)$, and $(k)$, it follows that the only possible edges in $G$ are:

- edges from $p_{1}$ to $P_{1}$;

- edges from $p_{n-1}$ to $P_{2}$;

- edges from $\operatorname{int}(P)$ to $x$ or to $y$;

- edges that are part of the $P_{2^{-}}$or $C_{4}$-bridges from $\operatorname{int}(P)$ to $x$ or to $y$;

- edges that are part of the $P_{2^{-}}$or $C_{4^{-}}$-bridges from $a_{1}$ or $b_{1}$ to $x$, and from $a_{s}$ or $b_{t}$ to $y$

- edges of the cycles $C_{x}$ and $C_{y}$.

If there are no $P_{2^{-}}$or $C_{4}$-bridges from $\operatorname{int}(P)$ to $x$ nor to $y$, then, just as in the proof of Case 2.1.2a, if $L^{\prime}\left(x, a_{1}\right)=C_{4}$ and $L^{\prime}\left(y, a_{s}\right)=C_{4}\left(\right.$ or $L^{\prime}\left(x, b_{1}\right)=C_{4}$ and $\left.L^{\prime}\left(y, b_{t}\right)=C_{4}\right)$, then $G \geqslant_{m} J_{3}$ (see old figure). Similarly, $L^{\prime}\left(x, a_{1}\right)=C_{4}$ and $L^{\prime}\left(y, b_{t}\right)=C_{4}\left(\right.$ or $L^{\prime}\left(x, b_{1}\right)=C_{4}$ and $\left.L^{\prime}\left(y, a_{s}\right)=C_{4}\right)$, then $G \geqslant_{m} J_{1}$ (see old figure). Therefore, for one of the sides, say the $x$-side, we must have that $x a_{1}$ and $x b_{1}$ are either simple edges, or edges subdivided once. Hence, $G-y$ is outerplanar, a contradiction.

Hence, there is a $P_{2^{-}}$or $C_{4}$-bridge from $\operatorname{int}(P)$ to $x$ or to $y$, but there cannot be such bridges to both $x$ and $y$, for otherwise $G$ would contain a $Q_{2}$-minor. Hence,

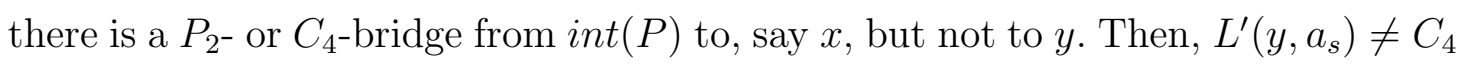
and $L^{\prime}\left(y, b_{t}\right) \neq C_{4}$, for otherwise $G \geqslant_{m} J_{5}$ (see figure below).

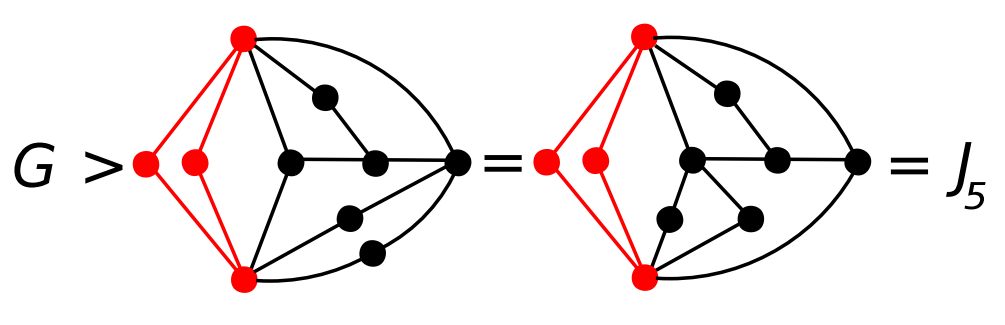


Therefore, $y a_{s}$ and $y b_{t}$ are either simple edges, or edges subdivided once. Hence, $G-x$ is outerplanar, a contradiction (see figure below).

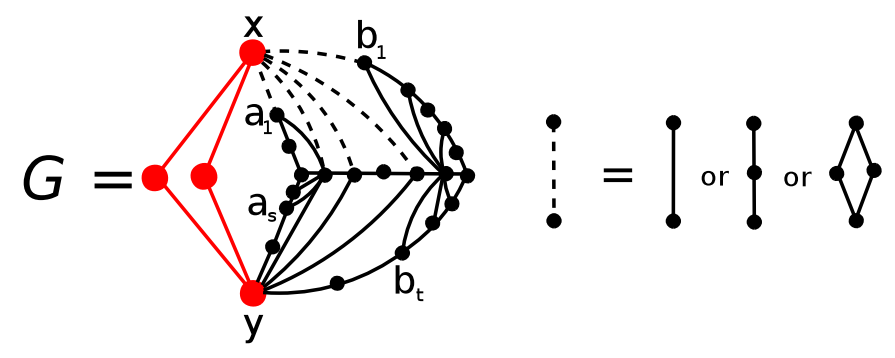

This concludes the proof of Case 2.1.2.

\subsubsection{Case 2.2}

Case 2.2: For each 2-separation, $L=P_{2}$;

Recall that a graph $H$ is internally 3-connected if it is 2-connected, and for every 2-cut $\{s, t\}, H-\{s, t\}$ has two connected components, one of which is a single vertex.

It follows from the Case 2.2 hypothesis that $G$ is internally 3 -connected. Let $(L, R)$ be a 2 -separation over vertices $\{x, y\}$ such that $L=P_{2}$. Let $v$ be the third (middle) vertex of $L$. Since $G$ is minor-minimal $\notin \mathcal{O}^{*}, G / v y$ has an apex vertex $a$ (i.e. $a$ such that $(G / v y)-a \in \mathcal{O}$ ). Note that $a \neq y$ and $a \neq x$, for otherwise $y$ (or $x$, respectively) is an apex vertex in $G$, a contradiction. Since $\operatorname{deg}(v)=2$, it follows that $G / v y$ is also internally 3 -connected. Hence, the only possible 1-separations in $(G / v y)-a$ are those that separate a pendant vertex. Call such 1-separations trivial. Therefore, $(G / v y)-a$ is 2-connected up to trivial 1-separations (pendant edges), and outerplanar.

Fix a planar embedding of $G$ so that all the vertices of $(G / v y)-a \in \mathcal{O}$ and $a$ are incident with the outer face (i.e. infinite face). Since $(G / v y)-a$ is 2-connected up to trivial 1-separations, it follows that all the vertices of $(G / v y)-a \in \mathcal{O}$ lie along a cycle $C$, except (possibly) for the vertices of degree 1 in $(G / v y)-a$ that 
are adjacent to some vertex of $C$. Note that such vertices have degree 2 in $G / v y$ (and in $G$ ), and that no two of them are adjacent to the same vertex $c$ of $C$, for otherwise $G$ has a 2-separation $\left(L^{\prime}, R^{\prime}\right)$ over $\{c, a\}$ such that $L^{\prime}=C_{4}$ or $L^{\prime} \notin \mathcal{O}$ and $R^{\prime} \notin \mathcal{O}$, contradicting the hypothesis of Case 2.2. Since $v \in G-a \notin \mathcal{O}$, it follows that $v$ is embedded in the interior of the disk bounded by $C$. Hence,

(a) The edges of $G$ are:

- edges of $C$;

- chords of $C$, that is, edges not in $E(C)$ with both endpoints in $C$ (note that such edges are embedded in the interior of the disk bounded by $C$ );

- edges $x v$ and $v y$, with $x, y \in V(C)$;

- edges with one endpoint in $C$ and the other at $a$ (or such edges subdivided once).

Also note that there are no two consecutive vertices in $C$ of degree 2 , since such vertices and their neighbors would induce a $P_{3}$ or a $C_{4}$ in $G$ giving rise to a 2-separation violating the hypothesis of Case 2.2 .

In this Case 2.2 , by a neighbor of $a$, we mean a vertex $u$ in $C$ such that $a u$ is actually an edge of $G$ or an edge subdivided once. As usual, we denote by $N(a)$ the set of neighbors of $a$. Since $x y \notin E(G)$, it follows that $G$ has vertices in both $C(x, y)$ and $C(y, x)$. Furthermore,

(b) a must have a neighbor in both $C(x, y)$ and $C(y, x)$.

For otherwise, $G$ has a 2 -separation over $\{x, y\}$ contradicting the hypothesis of Case 2.2.

Note that a chord must have both of its endpoints in $C[x, y]$ or $C[y, x]$. We say that two chords $c:=c_{1} c_{2}$ and $d:=d_{1} d_{2}$ are non-overlapping if their endpoints satisfy $c_{1}<c_{2} \leqslant d_{1}<d_{2}$ in the cyclic order of $C$. Two chords are said to be nested if they are not non-overlapping. It follows from $(a)$ that: 
$\left(b^{\prime}\right)$ If $c:=c_{1} c_{2}$ is a chord with $c_{1}<c_{2}$ (in the clockwise order restricted to $C[x, y]$ or $C[y, x])$, then $a$ has a neighbor in $C\left(c_{1}, c_{2}\right)$.

For otherwise, $G$ has a 2-separation over $\left\{c_{1}, c_{2}\right\}$ contradicting the hypothesis of Case 2.2. Also,

$\left(b^{\prime \prime}\right)$ Within a single segment $C[x, y]$ or $C[y, x]$, there are no non-overlapping chords (or equivalently, all the chords are nested).

Suppose that the chords $c:=c_{1} c_{2}$ and $d:=d_{1} d_{2}$ are non-overlapping with $c_{1}<c_{2} \leqslant d_{1}<d_{2}$ within, say $C[x, y]$. Then, by $\left(b^{\prime}\right), a$ has a neighbor in $C\left(c_{1}, c_{2}\right)$ and in $C\left(d_{1}, d_{2}\right)$, and by $(b)$, it has a neighbor in $C(y, x)$. Then, $G$ contains the following graph as a minor, which we label $Q_{1}$, and which can easily be verified to belong to $\mathbf{o b}\left(\mathcal{O}^{*}\right)$. This is a contradiction, since $G$ is minor-minimal $\notin \mathcal{O}^{*}$.

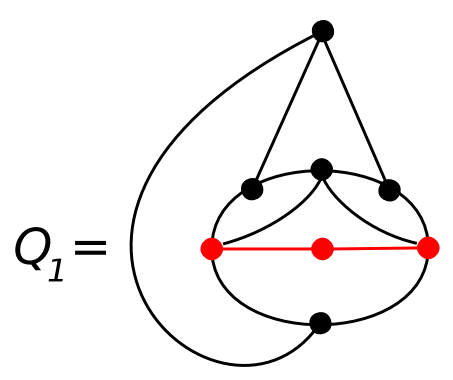

Case 2.2.1: For each 2-separation, $L=P_{2}$, and there exists such a 2-separation with $G-\{x, y\} \notin \mathcal{O}$;

We now further analyze the structure of $G$. By the hypothesis, $G-\{x, y\} \notin \mathcal{O}$, hence $G-\{x, y\}$ contains a $K$-subdivision as a subgraph, call it $K^{\prime}$. By $(a)$, it follows that $a$ is a cut-vertex in $G-\{x, y\}$, hence, without loss of generality, $K^{\prime}$ is a subgraph of $G-C[y, x]$. Let $C^{\prime}$ be the outer cycle of $K^{\prime}$. Then, $\left|V\left(C^{\prime}\right) \cap C(x, y)\right| \geqslant 2$, for otherwise if $u:=V\left(C^{\prime}\right) \cap C(x, y)$, then it follows by $(a)$ that $G$ has a 2separation $\left(L^{\prime}, R^{\prime}\right)$ over $\{a, u\}$ such that $L^{\prime} \notin \mathcal{O}$ and $R^{\prime} \notin \mathcal{O}$, contradicting the Case 2 hypothesis. 


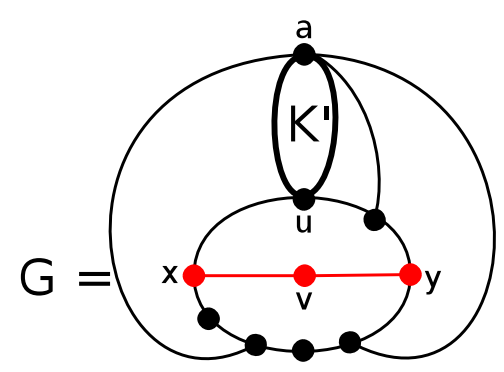

Let $s, t \in V\left(C^{\prime}\right) \cap C(x, y)$ be the first and last vertices, respectively, of $V\left(C^{\prime}\right) \cap$ $C(x, y)$ in the clockwise order of $C(x, y)$. Note that $s \neq x$ and $t \neq y$. Also, since $G$ does not contain two disjoint $K$-graphs, it follows that:

(c) $G$ does not have a chord with one endpoint in $C[x, s)$ and the other in $C(t, y]$.

It is straightforward to verify that the graphs in Figure 3.4 are minor-minimal $\notin \mathcal{O}^{*}$ satisfying the hypothesis of Case 2.2.1. We label them $H_{1}, H_{2}, H_{3}, H_{4}, H_{5}$. Hence $H_{i} \in \mathbf{o b}\left(\mathcal{O}^{*}\right)$ for $i=1, \ldots, 5$.
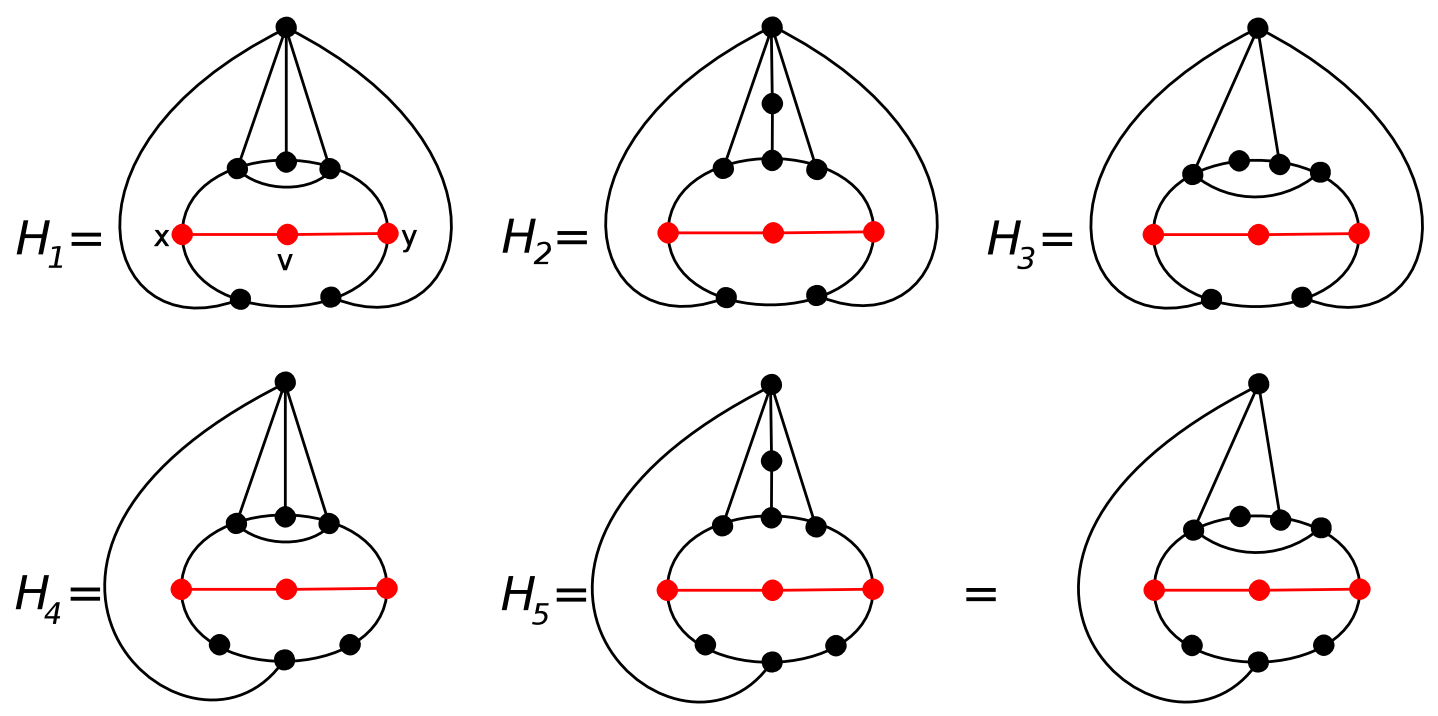

FIGURE 3.4. $\mathcal{H}$ family

Therefore, if $a$ has at least two neighbors in $C(y, x)$, or one neighbor $z \in C(y, x)$ and $C(y, z) \neq \varnothing$ and $C(z, x) \neq \varnothing$, then it is easy to verify that $G$ contains an $H_{i}$-minor for some $i=1, \ldots, 5$ (see figure below). 

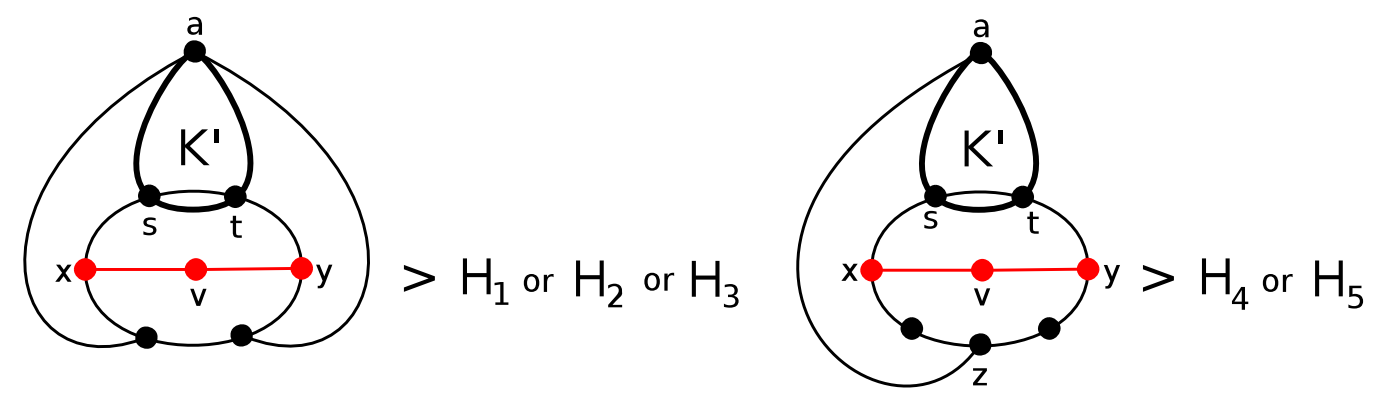

Hence, let $z$ be the only neighbor of $a$ in $C(y, x)$. We only need to consider two cases: either both $C(y, z)$ and $C(z, x)$ are empty, or one of them is empty, say $C(y, z)$, and the other is not.

First, suppose that $C(y, z)=\varnothing$ and $C(z, x) \neq \varnothing$. So $y z \in E(G)$. Then $G$ has the following structure as a subgraph:

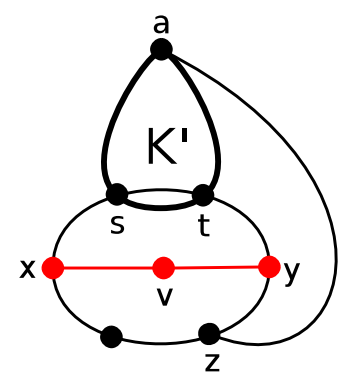

(d) In $G / y z$, the only apex vertex is $s$.

This is because an apex vertex in $G / y z$ must destroy both $K^{\prime}$ and the $\Theta$-graph with outer cycle $C$. Hence it must be a vertex in $V\left(C^{\prime}\right) \cap C(x, y)$. If $u \in C(s, t]$ is apex, then since $s, t$, and $a$ all lie on $C^{\prime}$, it follows that in $G / y z-u$ there is a path $P^{\prime}$ in $C^{\prime}$ from $a$ to $s$; this path, combined with the (possibly subdivided) edge ay $(=a z)$ and the path along $C$ from $y$ to $s$ form an outer cycle of a $\Theta$-graph with inner path $x, v, y$. Hence, $G / y z-u$ contains a $K_{2,3}$-subdivision, a contradiction. This proves $(d)$.

(e) $y(=z)$ is a cut-vertex in $G / y z-s$.

Note that there are no edges (or edges subdivided once) from $a$ to $C(z, x)$ in $G / y z$, since $z$ is the only neighbor of $a$ in $C(y, x)$ in $G$. Also, note that there are no 
edges (or edges subdivided once) from $a$ to $C[x, s)$ in $G / y z$, for otherwise $G / y z-s$ contains a $K_{2,3}$-subdivision, contradicting $(d)$. Finally, there are no chords from $C[x, s)$ to $C(s, t]$ in $G / y z$, for otherwise $G / y z-s$ contains a $K_{2,3}$-subdivision, a contradiction. These facts combined with $(c)$ imply $(e)$.

Therefore, it follows by $(e)$ that after uncontracting edge $y z$ in $G / y z-s$, the resulting graph $G-s$ is also outerplanar, a contradiction since $G \notin \mathcal{O}^{*}$.

Now consider the other case, that is: both $C(y, z)$ and $C(z, x)$ are empty (so that $y z, z x \in E(G))$. Recall that $z$ is the only neighbor of $a$ in $C(y, x)$. Then $G$ has the following structure as a subgraph:

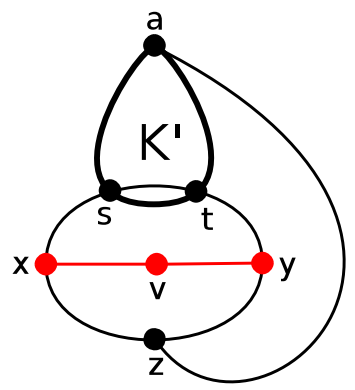

Similary to $(d)$, we obtain the following fact.

(f) In $G \backslash a z$, the only possible apex vertices are $s$ and $t$.

We use the above to prove the following key fact.

(g) One or both of the following hold:

(i) $x s$ is an edge of $G$ (or an edge subdivided once) and $\operatorname{deg}(x)=3$;

(ii) $y t$ is an edge of $G$ (or an edge subdivided once) and $\operatorname{deg}(y)=3$.

Note that if a vertex in $C(x, s)$ or $C(t, y)$ has degree $\geqslant 3$, then it is a neighbor of $a$ or an endpoint of a chord. Similarly, if $\operatorname{deg}(x) \geqslant 4$ or $\operatorname{deg}(y) \geqslant 4$, then $x$, respectively $y$, is a neighbor of $a$ or an endpoint of a chord. To prove $(g)$, we first note that $a$ does not have neighbors in both $C[x, s)$ and $C(t, y]$, for otherwise $G \backslash a z$ has no apex vertex (since neither $s$ nor $t$ is apex in $G \backslash a z$ ), a contradiction. Hence, by symmetry, $a$ has no neighbors in $C[x, s)$. Then, by $\left(b^{\prime}\right)$, there are no chords with 
both endpoints in $C[x, s]$. If $a$ has a neighbor in $C(t, y]$, then there are no chords with one endpoint in $C[x, s)$ and the other in $C(s, t]$, for otherwise $G \backslash a z$ has no apex vertex (note that the other endpoint cannot lie in $C(t, y]$ by $(c)$ ), and thus (i) holds. And if $a$ has no neighbors in $C(t, y]$ then, again by $\left(b^{\prime}\right)$, there are no chords with both endpoints in $C[t, y]$. Therefore, the only chords in $G$ are those with one endpoint in $C[x, s)$ and the other in $C(s, t]$ (in which case (ii) holds), or those with one endpoint in $C[s, t)$ and the other in $C(t, y]$ (in which case (i) holds), but not both, since two such chords would either cross or would be non-overlapping, violating $\left(b^{\prime \prime}\right)$. This proves $(g)$.

By symmetry, we have that (i) holds in $(g)$, so that $x s$ is an edge of $G$ (or an edge subdivided once, in which case denote the subdividing vertex by $w$ ). In the remainder of the proof, by $G / x s$ we mean the graph obtained from $G$ by contracting the path (of length 1 or 2) along $C$ from $s$ to $x$.

Similarly to $(d)$ and $(f)$, we obtain:

( $h$ ) In $G / x s$, the only apex vertex is $s(=x)$, unless (ii) in $(g)$ also holds, then $t$ may also be apex.

This is because if (ii) doesn't hold, then either $a$ has a neighbor in $C(t, y]$ or $G$ has a chord with one endpoint in $C[s, t)$ and the other in $C(t, y]$. And in either case $t$ is not apex in $G / x s$.

Note that $(G / x s)-s=G-\{x, s\}$ (or possibly $(G / x s)-s=G-\{x, w, s\}$ if $x s$ is subdivided). Re-embed the graph $(G / x s)-s \in \mathcal{O}$ (if necessary), so that all of its vertices are incident with the outer face. In $(G / x s)-s, \operatorname{deg}(z)=2$ and $\operatorname{deg}(v)=1$, hence edges $z y$ and $v y$ are also incident with the outer face. Since $y z$ is a simple edge, by putting $x$ (and possibly $w$ ) back in, we obtain an embedding of $G-s$ in which all the vertices are still incident with the outer face, hence $G-s$ is outerplanar, a contradiction (see figure below). 


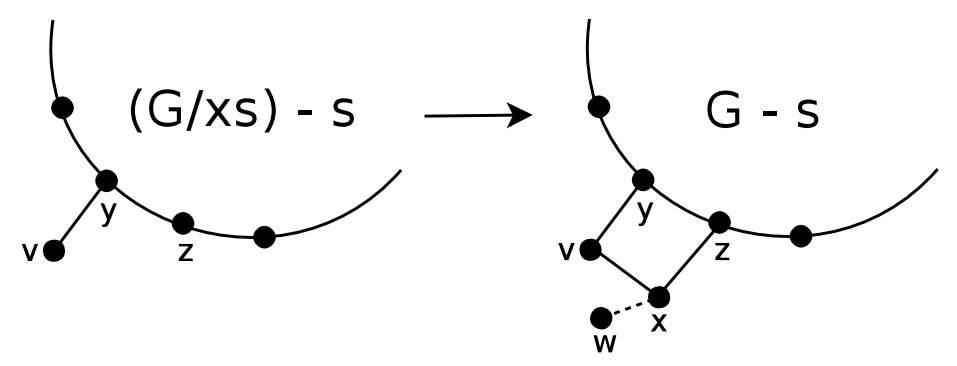

Finally, if $t$ is also apex in $G / x s$, then by the above, (ii) in $(g)$ also holds, so that $y t$ is an edge of $G$ (or an edge subdivided once, in which case denote the subdividing vertex by $u)$ and $\operatorname{deg}(y)=3$. Since $(G / x s)-t \in \mathcal{O}$, there is a face $f$ in the current embedding incident with all the vertices of $(G / x s)-t$. Since the path (of length 1 or 2 ) from $s$ to $x$ can be uncontracted along $C$, it follows that $f$ is also incident with all the vertices of $G-t$, a contradiction since $G \notin \mathcal{O}^{*}$ (see figure below).

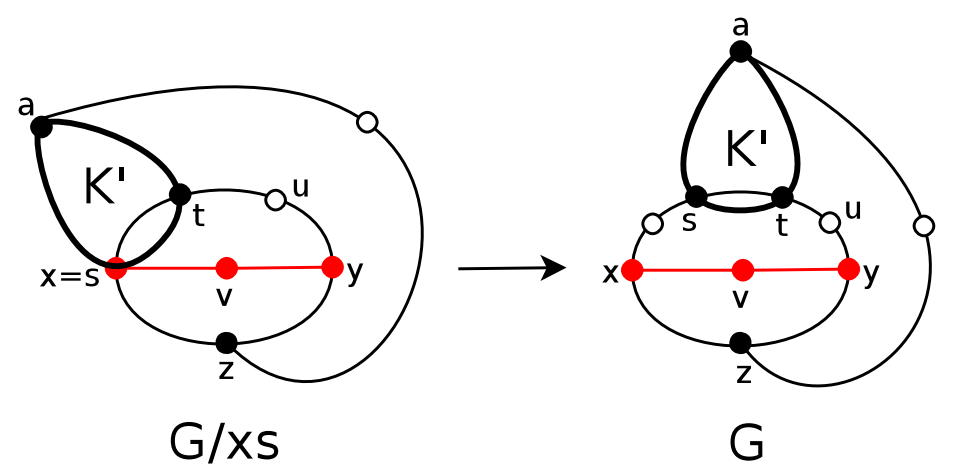

This concludes the proof of the subcase that both $C(y, z)$ and $C(z, x)$ are empty, as well as that of Case 2.1.2.

Case 2.2.2: For each 2-separation, $L=P_{2}$ and $G-\{x, y\} \in \mathcal{O}$.

It is straightforward to verify that the graphs in Figure 3.5 are minor-minimal $\notin \mathcal{O}^{*}$ satisfying the hypothesis of Case 2.2.2. We label them $Q_{1}, Q_{2}, Q_{3}, Q_{4}, Q_{5}$. Hence $Q_{i} \in \mathbf{o b}\left(\mathcal{O}^{*}\right)$ for $i=1, \ldots, 5$. 

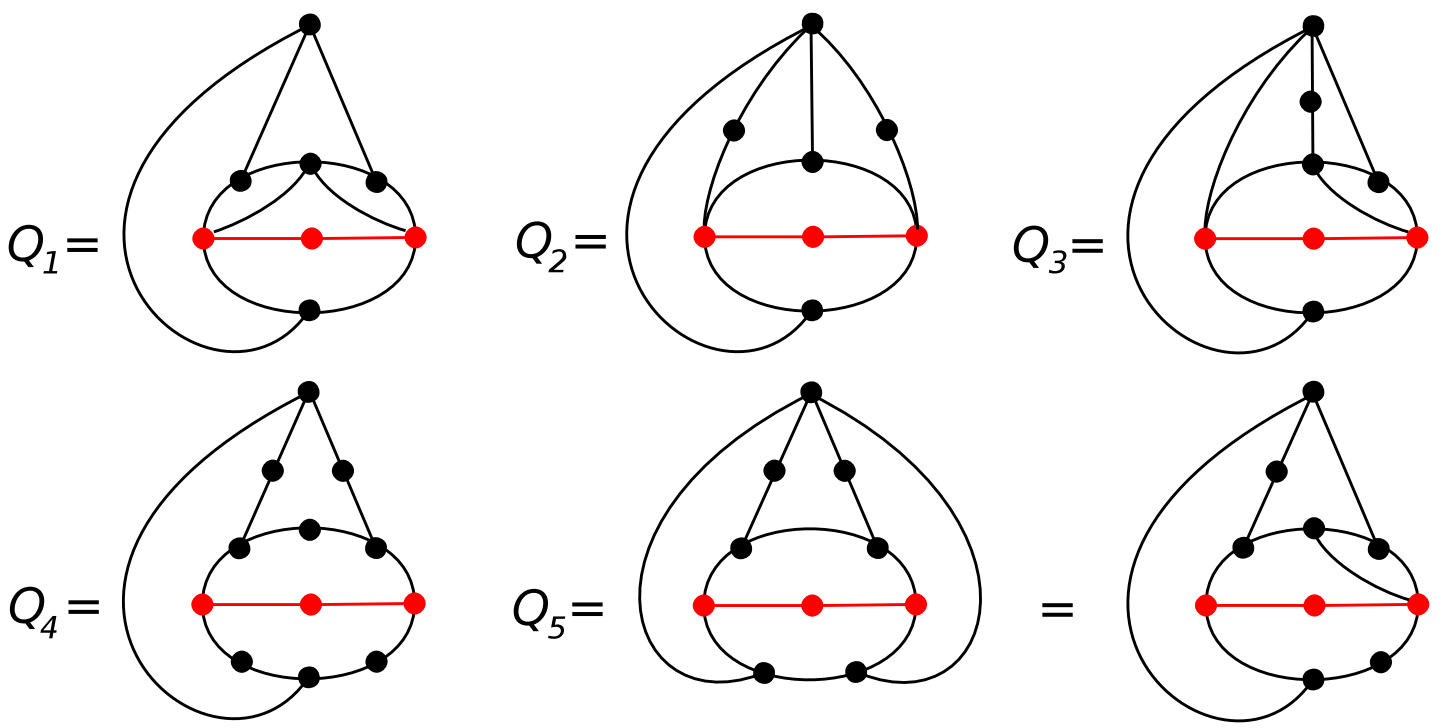

FIGURE 3.5. $\mathcal{Q}$ family

In the remainder of the proof, assume furthermore that $G \notin\left\{Q_{1}, Q_{2}, Q_{3}, Q_{4}, Q_{5}\right\}$. Observe that now:

(i) $\operatorname{deg}(a) \geqslant 3$

For otherwise, if $\operatorname{deg}(a)=2$, then let the two neighbors of $a$ be $a_{1}$ and $a_{2}$ (in $C(x, y)$ and $C(y, x)$, respectively, by $(b))$. Note that there is a chord with one endpoint in $C\left[x, a_{1}\right)$ and the other in $C\left(a_{1}, y\right]$, for otherwise, it follows by $(a)$ that $a_{1}$ is apex in $G$, a contradiction. Similarly, there is a chord with one endpoint in $C\left[y, a_{2}\right)$ and the other in $C\left(a_{2}, x\right]$, for otherwise, it follows by $(a)$ that $a_{2}$ is apex in $G$, a contradiction. Since $\operatorname{deg}(a)=2$, it follows that $G$ has a 2 -separation over $\left\{a_{1}, a_{2}\right\}$ such that $G-\left\{a_{1}, a_{2}\right\}$ contains a $K_{2,3}$-subdivision, contradicting the Case 2.2.2 hypothesis. This proves $(i)$.

We first consider the case the $G$ has no chords.

Case 2.2.2a: G has no chords.

$\underline{1,1 \text { Case: }}|N(a) \cap C(x, y)|=1$ and $|N(a) \cap C(y, x)|=1$. 
Then, there is a subdivided edge ay, for otherwise $x$ is apex. Also, there is a subdivided edge $a x$, for otherwise $y$ is apex, and hence $G \geqslant_{m} Q_{2}$, a contradiction. $\underline{2,1 \text { Case: }}|N(a) \cap C(x, y)|=2$ and $|N(a) \cap C(y, x)|=1$.

First suppose that $x, y \notin N(a)$. Let $a_{1} \in N(a) \cap C(y, x)$ and $a_{2}, a_{3} \in N(a) \cap$ $C(x, y)$ in the clockwise order around $C$. Then, there is a vertex in $C\left(a_{2}, a_{3}\right)$, for otherwise $a_{1}$ is apex. Edge $a a_{3}$ is subdivided, for otherwise $x$ is apex. Edge $a a_{2}$ is subdivided, for otherwise $y$ is apex. There is a vertex in $C\left(y, a_{1}\right)$, for otherwise $a_{2}$ is apex. Finally, there is a vertex in $C\left(a_{1}, x\right)$, for otherwise $a_{3}$ is apex, and hence $G \geqslant_{m} Q_{4}$, a contradiction.

Next, suppose that $x \in N(a)$, but $y \notin N(a)$. Then, edge $a a_{3}$ is subdivided, for otherwise $x$ is apex. Edge $a x$ is not subdivided, for otherwise $G \geqslant_{m} Q_{2}$, a contradiction. Edge $a a_{2}$ is subdivided, for otherwise $y$ is apex. Finally, there is a vertex in $C\left(a_{1}, x\right)$, for otherwise $a_{3}$ is apex, and hence $G \geqslant_{m} J_{1}$, a contradiction.

Finally, suppose that $x, y \in N(a)$. Then, at least one of $a a_{3}, a y$ is subdivided, for otherwise $x$ is apex. Also, at least one of $a a_{2}, a x$ is subdivided, for otherwise $y$ is apex. If $a a_{2}$ and $a a_{3}$ are, then $G \geqslant_{m} J_{1}$, a contradiction. If $a x$ and $a y$ are, then $G \geqslant_{m} Q_{2}$, a contradiction. Finally, if $a x$ and $a a_{3}$ are, or $a_{2}$ and $a y$ are, then again $G \geqslant_{m} Q_{2}$, a contradiction.

3+, 1 Case: $|N(a) \cap C(x, y)| \geqslant 3$ and $|N(a) \cap C(y, x)|=1$.

Let $a_{1} \in N(a) \cap C(y, x)$ and $a_{2}, a_{3} \in N(a) \cap C(x, y)$ be such that $a_{2}$ is the vertex in $N(a) \cap C(x, y)$ closest to $x$, and $a_{3}$ is the vertex in $N(a) \cap C(x, y)$ closest to $y$. Note that if $u \in N(a) \cap C\left(a_{2}, a_{3}\right)$, then edge $a u$ is not subdivided, for otherwise $G-\{x, y\}$ contains a $K_{2,3}$-subdivision, contradicting the Case 2.2.2 hypothesis.

Therefore, at least one of $a a_{3}$, ay (if $a y \in E(G)$ ) is subdivided, for otherwise $x$ is apex. Also, at least one of $a a_{2}$, $a x$ (if $a x \in E(G)$ ) is subdivided, for otherwise $y$ is apex. Hence, $G \geqslant_{m} Q_{2}$, a contradiction. 
2+,2+ Case: $|N(a) \cap C(x, y)| \geqslant 2$ and $|N(a) \cap C(y, x)| \geqslant 2$.

Let $a_{1}, a_{2} \in N(a) \cap C(y, x)$ and $a_{3}, a_{4} \in N(a) \cap C(x, y)$ be such that $a_{1}$ and $a_{4}$ are the two neighbors of $a$ closest to $y$, and $a_{2}$ and $a_{3}$ are the two neighbors of $a$ closest to $x$. Note that if $u \in N(a) \cap\left(C\left(a_{1}, a_{2}\right) \cup C\left(a_{3}, a_{4}\right)\right)$, then edge au is not subdivided, for otherwise $G-\{x, y\}$ contains a $K_{2,3}$-subdivision, contradicting the Case 2.2.2 hypothesis.

Therefore, at least one of $a a_{1}, a a_{4}$, ay (if $a y \in E(G)$ ) is subdivided, for otherwise $x$ is apex. Also, at least one of $a a_{2}, a a_{3}, a x$ (if $a x \in E(G)$ ) is subdivided, for otherwise $y$ is apex. Hence, it follows from these two facts that if $a y \in E(G)$ and it is subdivided, then $G \geqslant_{m} Q_{2}$, a contradiction. Similarly, if $a x \in E(G)$ and it is subdivided, then $G \geqslant_{m} Q_{2}$, a contradiction. Hence, if $a x \in E(G)$ or $a y \in E(G)$, then they are not subdivided. Finally, if $a a_{1}$ and $a a_{2}$ are, or if $a a_{3}$ and $a a_{4}$ are, then $G \geqslant_{m} Q_{5}$, a contradiction. And if $a a_{1}$ and $a a_{3}$ are, or if $a a_{2}$ and $a a_{4}$ are, then $G \geqslant_{m} Q_{2}$, a contradiction. This concludes the proof of Case 2.2.2a.

Case 2.2.2b: G has a chord.

We first strengthen $\left(b^{\prime}\right)$ to the following:

(j) If $c:=c_{1} c_{2}$ is a chord with $c_{1}<c_{2}$ (in the clockwise order restricted to $C[x, y]$ or $C[y, x])$, then $a$ has a neighbor in $C\left(c_{1}, c_{2}\right)$. Furthermore, for any such neighbor $w$, the edge $a w$ is not subdivided.

For otherwise, $G$ would have a 2-separation over $\{a, w\}$ contradicting the hypothesis of Case 2.2.2 (since $G-\{a, w\}$ would contain a $K_{2,3}$-subdivision).

We first prove the following two Lemmas that will greatly limit the structure of $G$. 
Lemma 3.1. Let $c=c_{1} c_{2}$, with $c_{1}, c_{2} \in C(x, y)$ in the clockwise order around $C$, be an innermost chord of $G$ (in the sense that there are no other chords with both endpoints in $\left.C\left[c_{1}, c_{2}\right]\right)$. Then a does not have two neighbors in $C\left(c_{1}, c_{2}\right)$.

Proof. Suppose that $a$ does have two neighbors $a_{1}, a_{2} \in C\left(c_{1}, c_{2}\right)$. By $(j)$, edges $a a_{1}$ and $a a_{2}$ are not subdivided. Also, $a$ does not have any other neighbors in $C(x, y)$, for otherwise $G-\{x, y\}$ would contain a $K_{4}$-subdivision, violating the hypothesis of Case 2.2.2. Also, $C\left(a_{1}, a_{2}\right)=\varnothing$, for otherwise $G-\{x, y\}$ would contain a $K_{2,3^{-}}$ subdivision, violating the hypothesis of Case 2.2.2. Note that possibly, edges $c_{1} a_{1}$ and $a_{2} c_{2}$ are subdivided once, but since $c$ is an innermost chord, there are no other vertices in $C\left(c_{1}, c_{2}\right)$. If $a$ has at least two neighbors in $C(y, x)$, then $G \geqslant_{m} Q_{5}$, a contradiction. Hence, let $z$ be the only neighbor of $a$ in $C(y, x)$.

We let $u$ be an apex vertex in $G \backslash a_{1} a_{2}$, and we assume that the graph $\left(G \backslash a_{1} a_{2}\right)$ $u \in \mathcal{O}$ is embedded in the plane with all of its vertices incident with the outer face. Note that $u \in\left\{z, c_{1}, c_{2}\right\}$, for otherwise: if $u \in\left\{a_{1}, a_{2}\right\}$, then clearly $u$ is apex in $G$, a contradiction; if $u \in\{a\} \cup C\left(c_{1}, a_{1}\right) \cup C\left(a_{2}, c_{2}\right)$, then $\left(G \backslash a_{1} a_{2}\right)-u$ contains a $K_{2,3}$-Subdivision; and if $u \in\{v\} \cup C\left(c_{2}, z\right) \cup C\left(z, c_{1}\right)$, then $\left(G \backslash a_{1} a_{2}\right)-u$ contains a $K_{4}$-subdivision.

If $u=z$, then the only neighbors of $a$ are $a_{1}, a_{2}$ and $z$ (because if $x$ or $y$ is a neighbor of $a$ then $\left(G \backslash a_{1} a_{2}\right)-z$ contains a $K_{2,3}$-subdivision $)$. Then, in $\left(G \backslash a_{1} a_{2}\right)-z$, $\operatorname{deg}(a)=2$, hence edges $a a_{1}$ and $a a_{2}$ are incident with the outer face, and by putting the edge $a_{1} a_{2}$ back in, we obtain an embedding of $G-z$ in which all the vertices are still incident with the outer face, hence $G-z$ is outerplanar, a contradiction.

Finally, suppose that $u=c_{1}$ (the case $u=c_{2}$ is symmetric). If $c_{1} a_{1}$ is subdivided once, then let $b$ be the subdividing vertex. Then, in $\left(G \backslash a_{1} a_{2}\right)-c_{1}$, then $\operatorname{deg}\left(a_{1}\right)=1$ (except if $c_{1} a_{1}$ is subdivided by $b$, then $\operatorname{deg}\left(a_{1}\right)=2$, but $a_{1}$ is adjacent to $b$ with 
$\operatorname{deg}(b)=1$, that is $a_{1} b$ is a pendant edge), and $\operatorname{deg}\left(a_{2}\right)=2$. Hence edges $a a_{2}$ and $a a_{1}$ (and possibly $a_{1} b$ ) are incident with the outer face, and since $a a_{2}$ is a simple edge, we can put edge $a_{1} a_{2}$ back in to obtain an embedding of $G-c_{1}$ in which all the vertices are still incident with the outer face, a contradiction.

Lemma 3.2. $G$ does not have a chord with both endpoints distinct from $x$ and $y$.

Proof. Suppose that $G$ does have a chord with endpoints $s, t \in C(x, y)$ in the clockwise order around $C$. We may assume, without loss of generality, that st is the innermost chord, in the sense that there are no other chords with both endpoints in $C[s, t]$. By $(j)$, there is a vertex $w \in N(a) \cap C(s, t)$ and the edge $a w$ is not subdivided. Also, by Lemma 3.1, $N(a) \cap C(s, t)=\{w\}$. Also, a does not have neighbors in both $C(x, s]$ and $C[t, y)$, for otherwise $G-\{x, y\}$ would contain a $K_{4^{-}}$ subdivision, violating the hypothesis of Case 2.2.2. Also, by $\left(b^{\prime \prime}\right), G$ does not have chords with both endpoints in $C[x, s]$ or both in $C[t, y]$. Let $z \in N(a) \cap C(y, x)$. First, we show that:

( $k$ ) Neither $s$ nor $t$ can be a neighbor of $a$.

By symmetry, we may assume that $t$ is a neighbor of $a$, so that $s$ is not. Then, $C(x, s] \cap N(a)=\varnothing$. Also, $C(w, t)=\varnothing$, for otherwise $G-\{x, y\}$ would contain a $K_{2,3}$-subdivision, violating the hypothesis of Case 2.2.2. Also, edges sw and ta are possibly subdivided once, but by choice of chord $c$, there are no other vertices in $C(s, t)$. Hence $G$ contains the following subgraph:

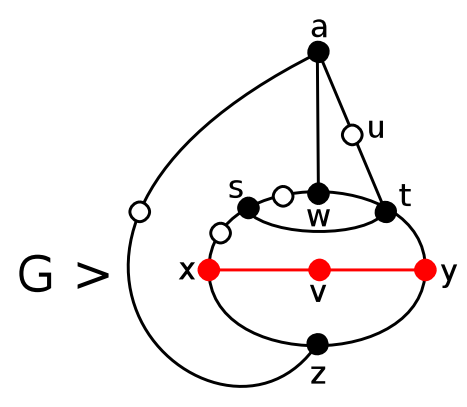


First, suppose that edge $t a$ is subdivided by vertex $u$. Then $C(t, y] \cap N(a)=$ $\varnothing$, for otherwise $G \geqslant_{m} Q_{3}$, a contradiction. For the same reason, we have that $(C(y, z) \cup C(z, x)) \cap N(a)=\varnothing$. Hence, the only neighbor of $a$ other than $z, w$ and $t$ is possibly $x$. Furthermore, if $a x \in E(G)$ then it is not subdivided for otherwise $G \geqslant_{m} Q_{2}$, a contradiction. Also, note that the remaining chords whose endpoints lie in $C[x, y]$ must have one of their endpoints at $t$, and the other in $C[x, s)$, for otherwise $\left(b^{\prime \prime}\right)$ is violated, or the subdivided edge $t a$ violates $(j)$. It follows from all of the above that if $C(z, x)=\varnothing$, then $t$ is apex in $G$, a contradiction. Hence $C(z, x) \neq \varnothing$. Then, if $a x \in E(G)$, then $G \geqslant_{m} J_{1}$ (by contracting $z$ to $y$, contracting $s$ to $x$, and deleting $w s$ ), a contradiction. Thus $a x \notin E(G)$. Therefore, since $C(w, t)=\varnothing$, if $G$ has no chords with one endpoint in $C[y, z)$ and the other in $C(z, x]$, then $z$ is apex in $G$, a contradiction. Hence, $G$ does have at least one such chord $c$. If $c$ has one endpoint in $C(z, x)$ and the other in $C[y, z)$, then $G \geqslant_{m} Q_{3}$ (by contracting $z$ to $a$, and $s$ to $x$ ), a contradiction. Hence, $c$ has one endpoint at $x$ and the other in $C(y, z)$, but then again $G \geqslant_{m} Q_{2}$ (by deleting st, contracting $z$ to $a$, contracting $s$ to $x$, and contracting $t$ to $y$ ), a contradiction. Thus we have shown that $t a$ is not subdivided, that is $t a \in E(G)$.

We let $p$ be an apex vertex in $G \backslash w t$, and we assume that the graph $(G \backslash w t)-p \in$ $\mathcal{O}$ is embedded in the plane with all of its vertices incident with the outer face. Note that $p \notin\{w, t\}$, for otherwise $p$ is apex in $G$, a contradiction. In fact, it is easy to see that if $p \notin\{z\} \cup C[x, s]$, then $p$ is not apex in $G \backslash w t$, a contradiction. $G$ and $G \backslash w t$ contain the following subgraphs, respectively: 

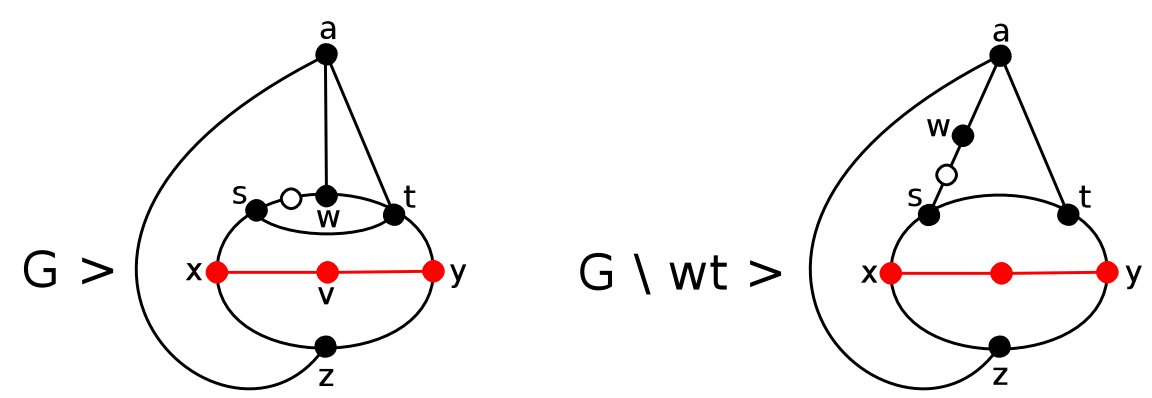

Suppose that $p=z$. Then, $a$ has no neighbors other than $w, t$, and $z$, for otherwise $(G \backslash w t)-p$ contains a $K_{4}$-subdivision, a contradiction. Therefore, in the graph $(G \backslash w t)-p, \operatorname{deg}(a)=2$, hence edges aw and at are incident with the outer face. Since at is a simple edge, we can put edge $w t$ back in, to obtain an embedding of $G-z$ in which all the vertices are still incident with the outer face, hence $G-z$ is outerplanar, a contradiction.

Therefore $p \in C[x, s]$. Recall from above that $C(x, s] \cap N(a)=\varnothing$. Note that there are no chords with one endpoint in $C[x, p)$ and the other in $C[t, y]$, for otherwise $(G \backslash w t)-p$ contains a $K_{2,3}$-subdivision, a contradiction. Also, if a chord has one endpoint in $C(p, s]$, then its other endpoint is $t$, for otherwise $(G \backslash w t)-p$ contains a $K_{4}$-subdivision, a contradiction. For simplicity, assume that $c=c_{1} t$ is the only such chord with $c_{1} \neq s$. If there is more than one such chord, the argument is similar. Also, note that edges $p c_{1}, c_{1} s$, and $s w$ may be subdivided once, but the subdividing vertices can be ignored for the purposes of this argument, as will be apparent soon. So for simplicity, we assume that $p c_{1}, c_{1} s$, and $s w$ are simple edges. By the observations above, it follows that in $(G \backslash w t)-p, \operatorname{deg}(w)=2$, and $\operatorname{deg}\left(c_{1}\right)=2$, hence edges $w a, w s, c_{1} s$ and $c_{1} t$ are incident with the outer face, which implies that edge st is not. Therefore, since in $(G \backslash w t)-p, \operatorname{deg}(s)=3$, it follows that by putting edge $w t$ back in, we obtain an embedding of $G-p$ in which all the vertices are still incident with the outer face, hence $G-p$ is outerplanar, a contradiction (see figure below). Finally, note that if edges $p c_{1}, c_{1} s$, and $s w$ are 
subdivided once, then its subdividing vertices are still incident with the outer face in the above embedding of $G-p$, since in the above argument edges $c_{1} s$ and $s w$ are incident with the outer face. This proves $(k)$.

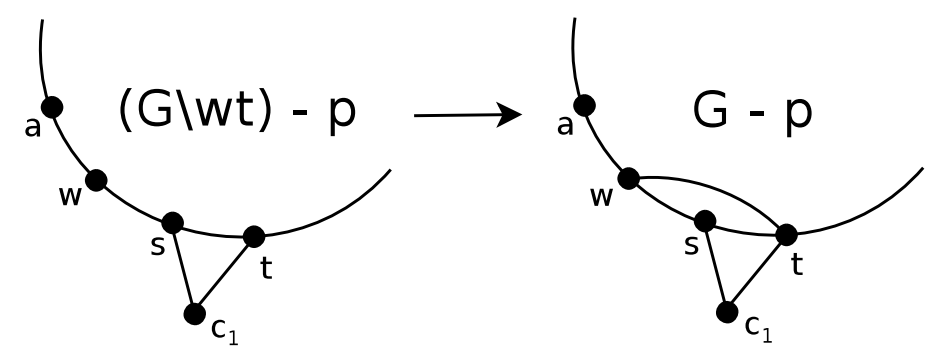

Therefore, neither $s$ nor $t$ is a neighbor of $a$. We now show furthermore:

(l) a does not have a neighbor in $C(x, s) \cup C(t, y)$.

By symmetry, suppose that $N(a) \cap C(t, y) \neq \varnothing$, so that $N(a) \cap C(x, s)=\varnothing$, and let $t^{\prime} \in N(a) \cap C(t, y)$. Then, all the chords that have an endpoint in $C(t, y)$ have the other endpoint at $x$, for otherwise $\left(b^{\prime \prime}\right)$ is violated, or $G-\{x, y\}$ contains a $K_{4}$-subdivision, a contradiction. Also, $C(w, t)=\varnothing$, for otherwise $G-\{x, y\}$ would contain a $K_{2,3}$-subdivision, violating the hypothesis of Case 2.2.2.

First, suppose that edge $t^{\prime} a$ is subdivided by vertex $u$. Then, $C\left(t, t^{\prime}\right) \cap N(a)=\varnothing$, for otherwise $G \geqslant_{m} Q_{2}$, a contradiction. Also, $C\left(t^{\prime}, y\right] \cap N(a)=\varnothing$, for otherwise $G \geqslant_{m} Q_{3}$, a contradiction. For the same reason, we have that $(C(y, z) \cup C(z, x)) \cap$ $N(a)=\varnothing$. Hence, the only neighbor of $a$ other than $z, w$ and $t^{\prime}$ is possibly $x$. Furthermore, if $a x \in E(G)$ then it is not subdivided for otherwise $G \geqslant_{m} Q_{2}$, a contradiction. Now consider what the remaining chords within $C[x, y]$ are. Note that a chord cannot have an endpoint in $C\left(t^{\prime}, y\right]$, since it would violate either $\left(b^{\prime \prime}\right)$ or $(j)$. And it cannot have an endpoint at $t$, since the other endpoint would be in $C[x, s)$, and $G$ would contain a $Q_{2}$-minor. Hence, all the remaining chords whose endpoints lie in $C[x, y]$ have an endpoint at $t^{\prime}$. It follows from all of the above 
that if $C(z, x)=\varnothing$, then $t^{\prime}$ is apex in $G$, a contradiction. Hence $C(z, x) \neq \varnothing$, and $G \geqslant_{m} Q_{5}$ (by contracting $s$ to $x$ and deleting all the chords incident with $t^{\prime}$ ), a contradiction. Thus we have shown that $t^{\prime} a$ is not subdivided, that is $t^{\prime} a \in E(G)$.

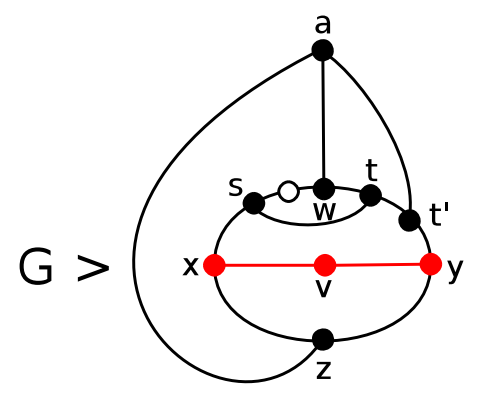

We will now proceed to show, in a sequence of steps, that the only possible chords with both endpoints in $C[x, y]$ other than st are the ones with one endpoint at $x$ and the other in $C\left[t^{\prime}, y\right)$. Recall from above that:

(1) All chords that have an endpoint in $C(t, y)$ have the other endpoint at $x$

(2) There is no chord with one endpoint at $t$ and the other in $C[x, s)$.

For otherwise, let $u \in C[x, s)$ be the other endpoint of such a chord, and choose $u$ to be the closest to $s$, in the sense that there are no chords with both endpoints in $C[u, t]$ other than st and $u t$. Note that, us and $s w$ are either edges of $G$ or edges subdivided once, but again we may assume, without loss of generality, that $u s$ and $s w$ are just simple edges. Let $p$ be an apex vertex in $G \backslash s t$. It is easy to see that $p \in C[x, u]$. If $p=u$, then there are no more chords with and endpoint at $t$, for otherwise $(G \backslash s t)-u$ contains a $K_{2,3}$-subdivision. Hence, in $(G \backslash s t)-u$, $\operatorname{deg}(t)=2$ and $\operatorname{deg}(s)=1$, hence edges $w t$ and $w s$ are incident with the outer face. Therefore, putting edge st back into this embedding, we obtain an outerplanar embedding of $G-u$, a contradiction. Therefore, we must have $p \in C[x, u)$. Also, if a chord has one endpoint in $C(p, u]$, then its other endpoint is $t$, for otherwise if the other endpoint is $y$, then $(G \backslash s t)-p$ contains a $K_{4}$-subdivision, a contradiction. For simplicity, assume that $c=c_{1} t$ is the only such chord with $c_{1} \neq s$. If there 
is more than one such chord, the argument is similar. Also, note that edges $p c_{1}$, $c_{1} u$, us and $s w$ may be subdivided once, but again we may assume, without loss of generality, that they are all just simple edges (since they will turn out to be incident with the outer face in $(G \backslash s t)-p)$. By the observations above, it follows that in $(G \backslash s t)-p, \operatorname{deg}(s)=2$, and $\operatorname{deg}\left(c_{1}\right)=2$, hence edges $s u, s w, c_{1} u$ and $c_{1} t$ are incident with the outer face, which implies that edge $u t$ is not. Therefore, since in $(G \backslash s t)-p, \operatorname{deg}(u)=3$, it follows that by putting edge $s t$ back in, we can embed $G-p$ so that all the vertices are still incident with the outer face, hence $G-p$ is outerplanar, a contradiction (see figure below). This proves (2).

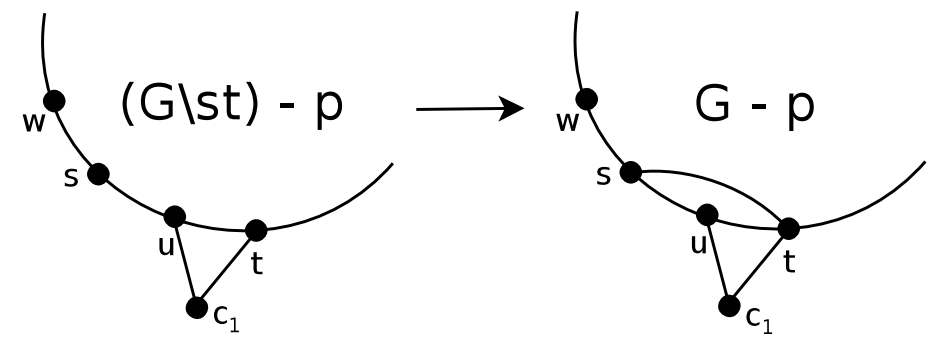

(3) There is no chord with one endpoint in $C\left(t, t^{\prime}\right)$ and the other at $x$.

Suppose the contrary, and let $u \in C\left(t, t^{\prime}\right)$ be the endpoint of such a chord. By (2), there is no chord with one endpoint at $t$ and the other in $C[x, s)$, hence $x s$ is an edge, or an edge subdivided once. Note that, $x s$ and $s w$ are either edges of $G$ or edges subdivided once, but again we may assume, without loss of generality, that $x s$ and $s w$ are just simple edges. Let $p$ be an apex vertex in $G \backslash s t$. It is easy to see that $p=x$. Hence, in $(G \backslash s t)-p, \operatorname{deg}(t)=2$ and $\operatorname{deg}(s)=1$, hence edges $w t$ and $w s$ are incident with the outer face. Therefore, putting edge $s t$ back into this embedding, we obtain an outerplanar embedding of $G-x$, a contradiction. This proves (3).

(4) There is no chord with one endpoint at $y$ and the other in $C(x, s]$

Suppose the contrary, and let $u \in C(x, s]$ be the endpoint of such a chord, and choose $u$ to be the closest to $s$, in the sense that there is no other chords with one 
endpoint at $y$ and the other in $C(u, s]$. Therefore, $u s$ and $s w$ are either edges of $G$ or edges subdivided once, but again we may assume, without loss of generality, that $u s$ and $s w$ are just simple edges. It is easy to see that $u$ is the only possible apex vertex in $G \backslash w t$. First, if $u \in C(x, s]$, then in $(G \backslash w t)-u, \operatorname{deg}(s)=2$, hence edges $s w$ and $s t$ are incident with the outer face. Therefore, putting edge $w t$ back into this embedding, we obtain an outerplanar embedding of $G-u$, a contradiction. Finally if $u=s$, then in $(G \backslash w t)-s, \operatorname{deg}\left(t^{\prime}\right)=2$ and $\operatorname{deg}(t)=1=\operatorname{deg}(w)$, hence edges $t t^{\prime}$, $t^{\prime} a$, and $a w$ are incident with the outer face. Therefore, since $a t^{\prime}$ is a simple edge, by putting edge $w t$ back into this embedding, we obtain an outerplanar embedding of $G-s$, a contradiction. This proves (4).

Therefore, it follows by (1) - (4) that:

(5) The only possible chord with both endpoints in $C[x, y]$ other than st are the ones with one endpoint at $x$ and the other in $C\left[t^{\prime}, y\right)$.

Hence, $x s$ and $s w$ are either edges of $G$ or edges subdivided once, but again we may assume, without loss of generality, that $x s$ and $s w$ are just simple edges. In the remainder of the proof of $(l)$, by $G / x s$ we mean the graph obtained from $G$ by contracting the path (of length 1 or 2 ) along $C$ from $s$ to $x$. Let $p$ be an apex vertex in $G / x s$. It is easy to see that $p=x$ or $p=t^{\prime}$. If $p=x$, then in $(G / x s)-x$, $\operatorname{deg}(w)=2=\operatorname{deg}(t)$, hence edge $w t$ is incident with the outer face. Therefore, by putting edges $w s$ and $s t$ back into this embedding, we obtain an outerplanar embedding of $G-x$, a contradiction. And if $p=t^{\prime}$, then observe the following facts. First, there are no chords with one endpoint at $x$ and the other in $C\left(t^{\prime}, y\right)$, therefore the only possible chord with both endpoints in $C[x, y]$ other than st is $x t^{\prime}$. Second, $a$ has no other neighbors, except possibly $x$, for otherwise $(G / x s)-t^{\prime}$ contains a $K_{4}$-subdivision. And if $x \in N(a)$, then $x a$ is not subdivided. Third, $C(z, x)=\varnothing$, and the only edges left in $G$ are chords from $x$ to $C(y, z)$. These 
facts account for all the edges of $G$. Hence $t^{\prime}$ is apex in $G$, a contradiction. This concludes the proof of $(l)$.

Therefore, $a$ does not have neighbors in $C(x, s) \cup C(t, y)$. It follows, by $\left(b^{\prime}\right)$, that there are no chords with both endpoints in $C[x, s]$ or both in $C[t, y]$. Again, we let $p$ be an apex vertex in $G / w a$. It follows from $(i)$ that besides $w$ and $z, a$ has another neighbor (in $C[y, x])$. Therefore $p \neq z$, since $(G / w a)-z$ contains a $K_{4}$-subdivision. In fact, it is easy to check that $p \in C[x, s] \cup C[t, y]$, for otherwise $(G / w a)-p$ contains a $K$-subdivision.
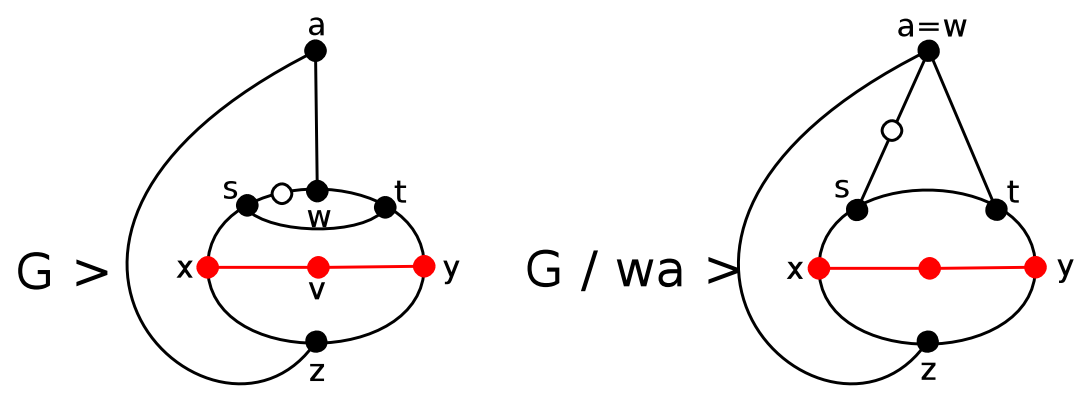

By symmetry, let $p \in C[x, s]$. First, if $p=s$, then all the chords whose endpoints lie in $C[x, y]$ have an endpoint at $s$, for otherwise $(G / w a)-s$ contains a $K_{2,3^{-}}$ subdivision, a contradiction. Thus, in $(G / w a)-s, \operatorname{deg}(t)=2$, hence edge $t a$ is incident with the outer face. Therefore, in the current embedding of $(G / w a)-s$, we can subdivide edge ta by $w$ to obtain an embedding of $G-s$ in which all the vertices are still incident with the outer face, hence $G-s$ is outerplanar, a contradiction.

Therefore, $p \in C[x, s)$. Then, by $(k)$ and $(l), a$ has no neighbors in $C(p, s]$. If a chord has an endpoint in $C(p, s]$, then its other endpoint is $t$, otherwise $(G / w a)-p$ contains a $K_{4}$-subdivision. For simplicity, assume that $c=c_{1} t$ is the only such chord with $c_{1} \neq s$. If there is more than one such chord, the argument is similar. Again, the edges $p c_{1}, c_{1} s$, and $s w$ may be subdivided once, but the subdividing vertices can be ignored for the purposes of this argument. So for simplicity, we assume 
that $p c_{1}, c_{1} s$, and $s w$ are simple edges. By the observations above, it follows that in $(G / w a)-p, \operatorname{deg}\left(c_{1}\right)=2$, hence edges $c_{1} s$ and $c_{1} t$ are incident with the outer face, which implies that edge st is not. Therefore, since in $(G / w a)-p, \operatorname{deg}(s)=3$, it follows that $s a$ is also incident with the outer face (and hence edge at is not, for otherwise the edges of the cycle $a, t, c_{1}, s, a$ are all incident with the outer face, which implies that those are all the vertices in $(G / w a)-p$, since $(G / w a)-p$ has no non-trivial 1-separations, a contradiction). Therefore, it follows that in the current embedding of $(G / w a)-p$, we can delete edge $s a$, subdivide edge at by vertex $w$, and add edge $w s$ and obtain an embedding of $G-p$ in which all the vertices are still incident with the outer face, hence $G-p$ is outerplanar, a contradiction (see figure below). This concludes the proof of the Lemma.

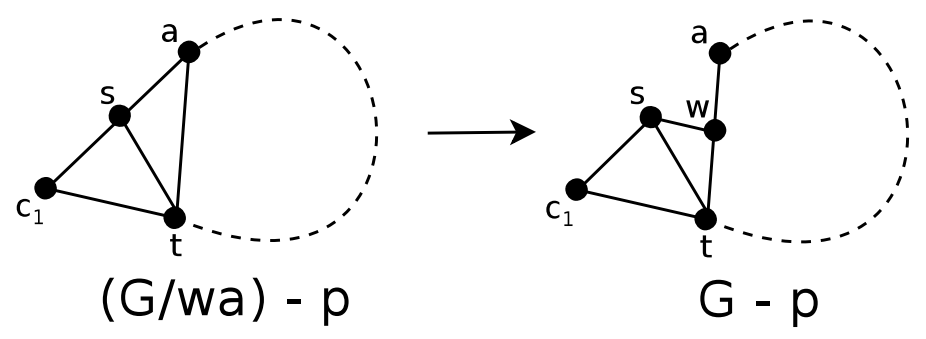

We now finish the proof of Case 2.2.2b and the entire connectivity-2 case. By Lemma 3.2 and $\left(b^{\prime \prime}\right)$, it follows that within each of the two segments $C[x, y]$ and $C[y, x]$ all the chords have an endpoint at $x$ or all the chords have an endpoint at $y$. We have three subcases:

Subcase $(i)$ : There are chords within $C[x, y]$ and within $C[y, x]$, and the ones within $C[x, y]$ have an endpoint at $y$, and the ones within $C[y, x]$ have an endpoint at $x$. Let $c_{1} y$ and $d_{1} x$ be innermost chords within $C[x, y]$ and $C[y, x]$, respectively. By $(j), a$ has a neighbor $w \in C\left(c_{1}, y\right)$, and a neighbor $z \in C\left(d_{1}, x\right)$, and edges $a w$ and $a z$ are not subdivided.

First, suppose that $a$ has a neighbor $u$ such that edge $a u$ is subdivided. Then, 
by $(j), u \notin C\left(c_{1}, y\right) \cup C\left(d_{1}, x\right)$. If $u \in C\left(x, c_{1}\right]$ or $u \in C\left(y, d_{1}\right]$, then $G \geqslant_{m} Q_{3}$ (by contracting $z a$ or $w a$, respectively), a contradiction. Therefore, $u \in\{x, y\}$, so by symmetry $u=x$. Since $G \ngtr_{m} Q_{2}$, it follows that $N(a) \cap(C(x, w) \cup C(w, y))=\varnothing$, $C(w, y)=\varnothing$, and if $y \in N(a)$, then $a y$ is not subdivided. Therefore, $x$ is apex in $G$, a contradiction.

Therefore, for all neighbors $u$ of $a, a u$ is a simple edge. Note that if $a$ has no neighbors in $C(x, w) \cup C(w, y)$ and $C(w, y)=\varnothing$, then $x$ is apex in $G$, a contradiction. Similarly, if $a$ has no neighbors in $C(y, z) \cup C(z, x)$ and $C(z, x)=\varnothing$, then $y$ is apex in $G$, a contradiction. Therefore, either $N(a) \cap(C(x, w) \cup C(w, y)) \neq \varnothing$ or $C(w, y) \neq \varnothing$; and either $N(a) \cap(C(y, z) \cup C(z, x)) \neq \varnothing$ or $C(z, x) \neq \varnothing$. It can easily be seen that any one of the four combination yields a $Q_{2}$-minor in $G$, a contradiction.

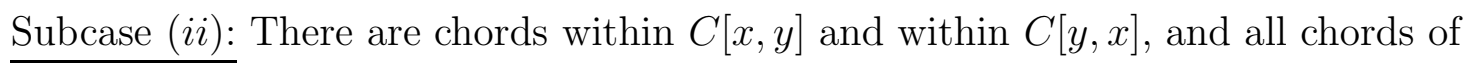
$G$ have an endpoint at $y$.

Let $c_{1} y$ and $d_{1} y$ be innermost chords within $C[x, y]$ and $C[y, x]$, respectively. By $(j), a$ has a neighbor $w \in C\left(c_{1}, y\right)$, and a neighbor $z \in C\left(y, d_{1}\right)$, and edges $a w$ and $a z$ are not subdivided.

Note that $a$ has a neighbor $u \neq y$ such that $a u$ is subdivided, for otherwise $y$ is apex in $G$, a contradiction. Then, by $(j), u \notin C\left(c_{1}, y\right) \cup C\left(y, d_{1}\right)$, hence $u \in C\left[x, c_{1}\right] \cup$ $C\left[d_{1}, x\right]$. By symmetry, we only need to consider $u \in C\left[x, c_{1}\right]$. First, if $u=x$, then since $G \ngtr_{m} Q_{2}$, it follows that $N(a) \cap(C(x, w) \cup C(w, y) \cup C(y, z) \cup C(z, x))=\varnothing$, $C(w, y) \cup C(y, z)=\varnothing$, and if $y \in N(a)$, then $a y$ is not subdivided. Therefore, $x$ is apex in $G$, a contradiction. Second, if $u \in C\left(x, c_{1}\right)$, then since $G \ngtr_{m} Q_{3}$, it follows that $N(a) \cap C(z, u)=\varnothing$. Also, since $G \ngtr_{m} Q_{2}$, it follows that $N(a) \cap C(u, w)=\varnothing$, and $C(w, y) \cup C(y, z)=\varnothing$, and if $y \in N(a)$, then ay is not subdivided. Therefore, $u$ is apex in $G$, a contradiction. Therefore we must have $u=c_{1}$. Again, since 
$G \ngtr_{m} Q_{3}$, it follows that $N(a) \cap C(z, u)=\varnothing$. And, since $G \ngtr_{m} Q_{2}$, it follows that $C(y, z)=\varnothing$, and if $y \in N(a)$, then ay is not subdivided. Therefore, $u$ is apex in $G$, a contradiction.

Subcase (iii): All the chords of $G$ lie within $C[x, y]$ and they all have an endpoint at $y$.

Let $c_{1} y$ be an innermost chord within $C[x, y]$. By $(j)$, a has a neighbor $w \in$ $C\left(c_{1}, y\right)$, and edge $a w$ is not subdivided.

Note that $a$ has a neighbor $u \neq y$ such that $a u$ is subdivided, for otherwise $y$ is apex in $G$, a contradiction. Then, by $(j), u \notin C\left(c_{1}, y\right)$. Let $z \in C(y, x)$ be the neighbor of a closest to $y$, in the sense that $y z$ is an edge of $G$ or an edge subdivided once. Then $u \in C\left(z, c_{1}\right]$, for otherwise $y$ is apex in $G$. First, if $u \in C(z, x]$, then $N(a) \cap C(u, x)=\varnothing$, for otherwise $G-\{x, y\}$ contains a $K_{2,3^{-}}$ subdivision, a contradiction. Also, since $G \ngtr m Q_{2}$, it follows that $N(a) \cap C(x, w)=$ $\varnothing, C(w, y)=\varnothing$, and if $y \in N(a)$, then ay is not subdivided. Therefore, $x$ is apex in $G$, a contradiction. Second, if $u \in C\left(x, c_{1}\right)$, then since $G \ngtr_{m} Q_{3}$, it follows that $N(a) \cap C(z, u)=\varnothing$. Also, since $G \ngtr_{m} Q_{5}$, we have that $C(y, z)=\varnothing$. And, since $G \ngtr_{m} Q_{2}$, it follows that $N(a) \cap C(u, w)=\varnothing$, and $C(w, y)=\varnothing$, and if $y \in N(a)$, then $a y$ is not subdivided. Therefore, $u$ is apex in $G$, a contradiction. Therefore, we must have $u=c_{1}$. Hence, $c_{1} y$ is the only chord in $G$, for otherwise $(j)$ would be violated. Again, since $G \ngtr_{m} Q_{3}$, it follows that $N(a) \cap C(z, u)=\varnothing$. Hence, $z x$ and $x c_{1}(=x u)$ are either edges of $G$ or edges subdivided once. Also, since $G \ngtr_{m} Q_{2}$, it follows that if $y \in N(a)$, then ay is not subdivided. Hence, $C(y, z) \neq \varnothing$, for otherwise $u$ is apex in $G$. Finally, since $G \ngtr_{m} J_{1}$, it follows that $C(u, w)=\varnothing$ and $N(a) \cap C(w, y]=\varnothing$, and hence $z$ is apex in $G$, a contradiction.

This concludes the proof of Case 2.2.2b and the entire connectivity-2 case. 


\section{Chapter 4 \\ Connectivity Three}

In this section, we let $G$ be 3 -connected graph in $\mathbf{o b}\left(\mathcal{O}^{*}\right)-\left\{K_{5}, K_{3,3}\right.$, Oct, $\left.Q\right\}$, and show that this set is actually empty.

We will make use of the following fundamental Theorem of Whitney from 1933, which can be found in [3].

Theorem 4.1 (Whitney). Every simple 3-connected planar graph has a unique planar embedding.

Whitney's Theorem has an immediate consequence for outerplanar graphs.

Corollary 4.2. Every simple 2-connected outerplanar graph has a unique outerplanar embedding.

We will also use the following basic lemma about 3-connected graphs (see [4]).

Lemma 4.3. If $G$ is 3-connected and $|V(G)| \geqslant 5$, then $G$ has an edge e such that G/e is also 3-connected.

Such an edge is called contractible. We denote by $v_{x y}$ the new vertex obtained by contracting edge $x y$. Since $G$ is minor-minimal $\notin \mathcal{O}^{*}$, there are two possibilities:

Case 1: There exists a contractible edge $x y \in E(G)$ such that $v_{x y}$ is not apex in $G / x y$ (and hence, there exists an apex vertex $a \neq v_{x y}$ in $G / x y$ ).

Case 2: For every contractible edge $x y \in E(G), v_{x y}$ is an apex vertex in $G / x y$.

\subsection{Case 1}

Let $x y$ be an edge and $a$ be a vertex that satisfy the hypothesis. Then, $(G / x y)-a \in$ $\mathcal{O}$ is 2 -connected. Since $G$ is 3 -connected, it has a unique planar embedding by Whitney's Theorem. Since $(G / x y)-a \in \mathcal{O}$ is 2 -connected, it follows by Corollary 
4.2 , that restricting this embedding to $(G / x y)-a$, we have that all the vertices of $(G / x y)-a$ lie on a cycle $C^{\prime}$ and are incident with the outer face. Since $G-a \notin \mathcal{O}$, it follows that $x$ or $y$, say $x$, is embedded in the interior of the disk bounded by $C$, where $C \subseteq G$ is the cycle isomorphic to $C^{\prime}$, and the corresponding isomorphism $\phi: V\left(C^{\prime}\right) \rightarrow V(C)$ is the identity map on $V\left(C^{\prime}\right)-v_{x y}$ and $\phi\left(v_{x y}\right)=y$.

Let $u_{1}, u_{2}, \ldots, u_{n} \in V(C)(n \geqslant 3)$ be the neighbors of $x$ in the clockwise order around $C$. For $i=1, \ldots, n$, let $S_{i}:=C\left[u_{i}, u_{i+1}\right]$, where $S_{n}$ is understood to be $C\left[u_{n}, u_{1}\right]$. We call the $S_{i}$ 's the segments of $C$. We call $u_{i}$ 's the endpoint vertices of the segments and the vertices in $C\left(u_{i}, u_{i+1}\right)$ for $i=1, \ldots, n$, the interior vertices of the segments. Two segments of $S_{i}$ and $S_{j}$ are said to be consecutive if $|i-j|=1$ (where $S_{n+1}=S_{1}$ ). We observe the following facts.

(a) The edges of $G$ are:

- edges of $C$;

- edges $x u_{i}$ for $i=1, \ldots, n$;

- chords of $C$, that is, edges not in $E(C)$ with both endpoints in a single segment of $C$ (note that such edges are embedded in the interior of the disk bounded by $C)$;

- edges with one endpoint in $C$ and the other at $a$.

It follows by the above that:

(b) Interior vertices of the segments are either endpoints of chords or neighbors of $a$.

(c) For every chord $c_{1} c_{2}$ in $G$ with $c_{1}<c_{2}$ (in the clockwise order restricted to the segment containing $\left.c_{1} c_{2}\right), a$ has a neighbor in $C\left(c_{1}, c_{2}\right)$ (by 3 -connectivity of $G$ ).

Let $N(a):=N_{G}(a)$. We now prove the following Lemma.

Lemma 4.4. $N(a)$ is covered by exactly two consecutive segments of $C$. 
Proof. First, we show that $N(a)$ is covered by exactly two segments of $C$. If there are four internally disjoint paths from $a$ to $x$, then the subgraph of $G$ formed from the union of those paths and $C$ contains an Oct-minor, a contradiction.

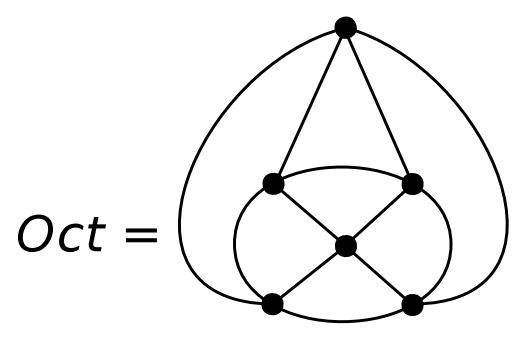

Therefore, by Menger's Theorem and the fact that $G$ is 3-connected, it follows that $G$ has a 3 -cut separating $a$ and $x$. By $(a)$ above, it follows that this 3 -cut is a subset of $V(C)$, and therefore at least one of $a$ or $x$ has degree 3. Let $u \in\{a, x\}$ be such that $\operatorname{deg}_{G}(u)=3$, and let $v \in\{a, x\}-\{u\}$. The three neighbors of $u$ divide $C$ into three segments. If all three segments contain interior vertices that are in $N(v)$, then $G$ contains a $Q$-minor, a contradiction

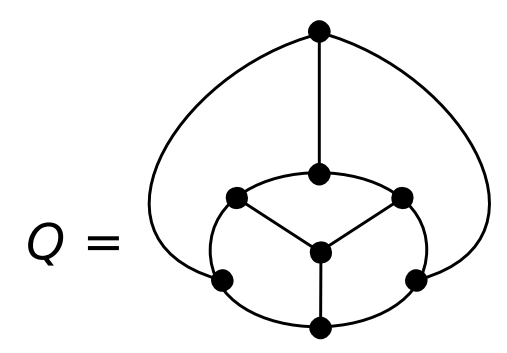

Hence, one segment does not contain any interior vertices that are in $N(v)$. Then, if $u=x$ then we are done. And similarly, if $u=a$ then we are done. Hence, we have shown that $N(a)$ is covered by exactly two segments of $C$.

Furthermore, the two segments that cover $N(a)$ are consecutive. Suppose not, and let $S_{i}$ and $S_{j}$ be the two segments that cover $N(a)$ with $|i-j|>1$. If both of them contain at least two neighbors of $a$, then two of those neighbors in each segment can be contracted to four distinct endpoint vertices and thus $G \geqslant_{m}$ Oct, a contradiction. Hence, one of them, say $S_{i}$, contains only one neighbor of $a$, call it $n_{1}$. Since $\operatorname{deg}(a) \geqslant 3, S_{j}$ must contain at least two neighbors of $a$ : let $n_{2}$ be the 
closest one to $u_{j}$, and $n_{3}$ be the closest one to $u_{j+1}$.

Suppose $n_{1}$ is an endpoint vertex, so that $n_{1}=u_{i}$ or $u_{i+1}$. Note that in this case $\operatorname{deg}(x) \geqslant 5$, for otherwise two consecutive segments cover $N(a)$. Then, since $G \notin \mathcal{O}^{*}$, it follows that $C\left(n_{2}, n_{3}\right) \neq \varnothing$ (for otherwise $n_{1}$ is an apex vertex). But then, $G \geqslant_{m} Q_{1}$, a contradiction (by deleting edge $n_{1} x$ and contracting $n_{2}$ to $u_{j}$, and $n_{3}$ to $\left.u_{j+1}\right)$.

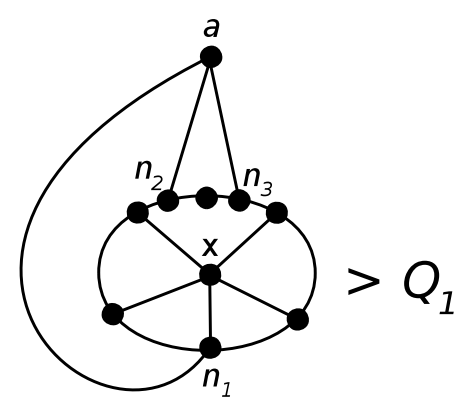

Therefore, $n_{1}$ must be an interior vertex, so $n_{1} \in C\left(u_{i}, u_{i+1}\right)$. Again, since $G \notin$ $\mathcal{O}^{*}$, there is a vertex in $C\left(n_{2}, n_{3}\right)$, or there is a chord with one endpoint in $C\left[u_{i}, n_{1}\right)$ and the other in $C\left(n_{1}, u_{i+1}\right]$ (for otherwise $n_{1}$ is an apex vertex). In the first case, $G \geqslant_{m} Q_{1}$ (just like above), a contradiction. And in the second case, $G \geqslant_{m}$ Oct, a contradiction (by contracting edge $n_{1} a$ ). This proves the Lemma.

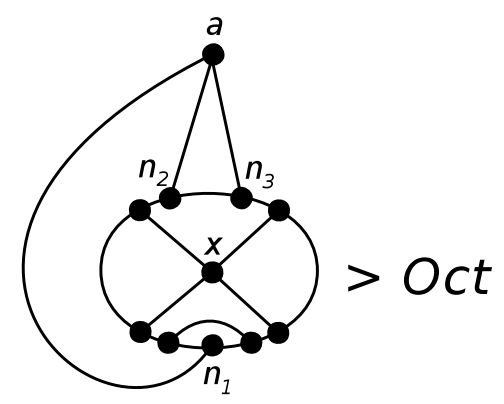

We now show that $C$ actually has exactly three segments.

Lemma 4.5. $C$ has exactly three segments, or equivalently deg $(x)=3$, or equivalently $n=3$. 
Proof. By Lemma 4.4, we may assume that $N(a)$ is covered by $S_{1}$ and $S_{2}$. Since interior vertices are either endpoints of chords or neighbors of $a$, it follows by $(b)$ and $(c)$ that $C\left(u_{i}, u_{i+1}\right)=\varnothing$ for $i=3,4, \ldots, n\left(\right.$ where $\left.u_{n+1}=u_{1}\right)$.

Suppose that $n \geqslant 4$. By Lemma 4.4, it follows that $a$ has neighbors in $C\left[u_{1}, u_{2}\right)$ and $C\left(u_{2}, u_{3}\right]$. Therefore, in the graph $G \backslash x u_{4}$, none of the vertices $a, u_{2}, x, u_{4}$ can be apex (since the deletion of any one of them still leaves a $K_{2,3}$-subdivision as a subgraph). Let $s$ be an apex vertex in $G \backslash x u_{4}$. Then $s \in V(C)$. Therefore, the unique embedding of $G$ restricted to the graph $\left(G \backslash x u_{4}\right)-s \in \mathcal{O}$ is an embedding in which all the vertices (including $x$ ) are incident with the outer face. By adding edge $x u_{4}$ to this embedding, we obtain an embedding of $G-s$ in which all the vertices are incident with the outer face, a contradiction.

Hence, we have shown that for $i=3,4, \ldots, n x u_{i} \notin E(G)$, therefore, by 3connectivity of $G$, it follows that $C$ has exactly three segments.

Hence $G$ has the following general structure:

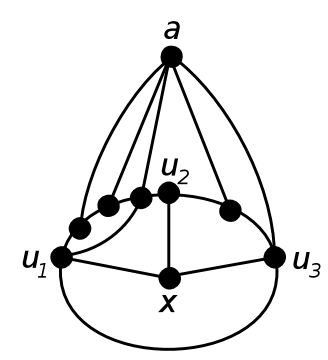

Therefore, let $S_{1}$ and $S_{2}$ cover $N(a)$. It follows by $(b)$, and $(c)$ that $C\left(u_{3}, u_{1}\right)=\varnothing$ (that is $u_{3} u_{1} \in E(G)$ ). Also, similarly to $\left(b^{\prime \prime}\right)$ of Case 2.2 , since $G \ngtr_{m} Q_{1}$, we have that

(d) Within a single segment $S_{1}$ or $S_{2}$, there are no non-overlapping chords (or equivalently, all the chords are nested).

We say that segment $S_{1}$ (respectively $S_{2}$ ) is of type-one, if $\{z\}:=N(a) \cap C\left[u_{1}, u_{2}\right)$ with $z \neq u_{1}$, and $C\left(z, u_{2}\right) \neq \varnothing$ (respectively, $\{w\}:=N(a) \cap C\left(u_{2}, u_{3}\right]$ with $w \neq u_{3}$, 
and $\left.C\left(u_{2}, w\right) \neq \varnothing\right)$. And we say that $S_{1}$ (respectively $S_{2}$ ) is of type-two, if $\mid N(a) \cap$ $C\left[u_{1}, u_{2}\right) \mid \geqslant 2$ (respectively $\left|N(a) \cap C\left(u_{2}, u_{3}\right]\right| \geqslant 2$ ). Note that if $S_{1}$ (respectively $\left.S_{2}\right)$ is not of type-one nor type-two, then $\{z\}:=N(a) \cap C\left[u_{1}, u_{2}\right)$ and $z u_{2} \in E(C)$ (respectively $\{w\}:=N(a) \cap C\left(u_{2}, u_{3}\right]$ and $u_{2} w \in E(C)$ ). Finally, note that at least one of $S_{1}$ or $S_{2}$ is of type-one or type-two, for otherwise $u_{2}$ is apex in $G$.

Hence, there are two subcases:

Case 1.1: Each of $S_{1}$ and $S_{2}$ is of type-one or type-two.

Suppose that one of the segments, say $S_{2}$ is of type-one. Then, $\{w\}:=N(a) \cap$ $C\left(u_{2}, u_{3}\right]$ with $w \neq u_{3}$, and $C\left(u_{2}, w\right) \neq \varnothing$. Hence, it follows by $(b)$, that there is a chord with one endpoint $c_{1} \in C\left(u_{2}, w\right)$, and the other $c_{2} \in C\left(w, u_{3}\right]$. Choose $c_{1}$ and $c_{2}$ so that the chord $c_{1} c_{2}$ is innermost. Then by $(d)$, all other chords in $S_{2}$ have one endpoint in $C\left[u_{2}, c_{1}\right]$ and the other in $C\left[c_{2}, u_{3}\right]$. However, since $S_{1}$ is of type-one or type-two, we either have $\{z\}:=N(a) \cap C\left[u_{1}, u_{2}\right)$ with $z \neq u_{1}$, and $C\left(z, u_{2}\right) \neq \varnothing$ (which by $(b)$ implies that there is a chord with one endpoint in $C\left[u_{1}, z\right)$ and the other in $\left.C\left(z, u_{2}\right)\right)$, or $\left|N(a) \cap C\left[u_{1}, u_{2}\right)\right| \geqslant 2$. This implies that the only other chords in $S_{2}$ that do not have an endpoint at $u_{2}$ (that is, those that do have an endpoint in $\left.C\left(u_{2}, c_{1}\right]\right)$ have an endpoint at $c_{2}$, for otherwise $G \geqslant_{m} Q_{1}$ (by contracting $w a$ and $z a$ if necessary, see figure below).

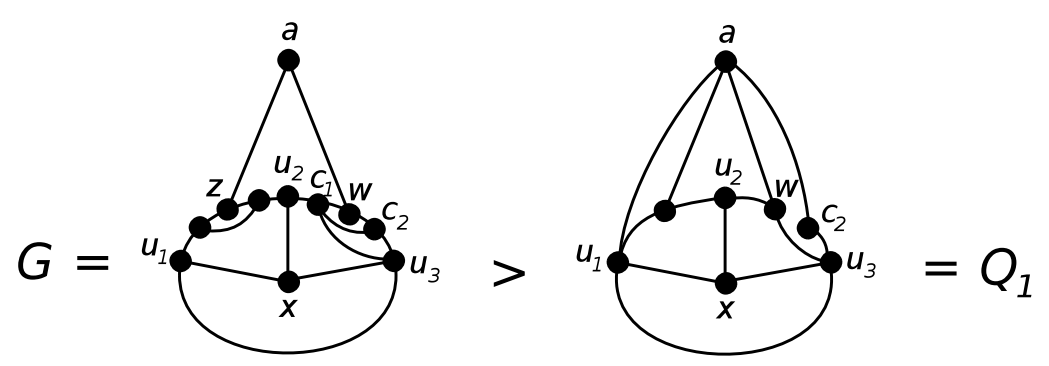

Therefore, $u_{2}$ is apex in $G$, a contradiction.

Similarly, suppose that for one of the segments, say $S_{1}$, is of type-two. Then, $\left|N(a) \cap C\left[u_{1}, u_{2}\right)\right| \geqslant 2$. If there are chords with endpoints distinct from $u_{2}$ in 
$S_{1}$, then let $d_{1} d_{2}$, with $d_{1}<d_{2}$ in the cyclic order of $C$, be an innermost chord of $S_{1}$ with $d_{2} \neq u_{2}$, and let $z \in N(a) \cap C\left(d_{1}, d_{2}\right)$. Then again, since $S_{2}$ is of type-one or type-two, we either have $\{w\}:=N(a) \cap C\left(u_{2}, u_{3}\right]$ with $w \neq u_{3}$, and $C\left(u_{2}, w\right) \neq \varnothing$ (which by $(b)$ implies that there is a chord with one endpoint in $C\left(u_{2}, w\right)$ and the other in $\left.C\left(w, u_{3}\right]\right)$, or $\left|N(a) \cap C\left(u_{2}, u_{3}\right]\right| \geqslant 2$. This implies that the only other chords in $S_{1}$ that do not have an endpoint at $u_{2}$ (that is, those that do have an endpoint in $\left.C\left[d_{1}, u_{2}\right)\right)$ have an endpoint at $d_{1}$, for otherwise $G \geqslant_{m} Q_{1}$. Furthermore, $N(a) \cap\left(C\left(d_{1}, z\right) \cup C\left(z, u_{2}\right)\right)=\varnothing$, for otherwise $G \geqslant_{m} Q_{1}$ as above. Therefore again, $u_{2}$ is apex in $G$, a contradiction.

Case 1.2: Exactly one of the segments $S_{1}$ or $S_{2}$ is of type-one or type-two.

By symmetry, suppose that $S_{2}$ is not of type-one nor type-two, and that $S_{1}$ is. Then, $\{w\}:=N(a) \cap C\left(u_{2}, u_{3}\right]$ and $u_{2} w \in E(C)$. We divide this case into two subcases depending on whether $u_{1} u_{2}$ is an edge of $G$.

Case 1.2.1: $u_{1} u_{2} \notin E(G)$

Let $s$ be an apex vertex in $G \backslash x u_{3}$, and we assume that the graph $\left(G \backslash x u_{3}\right)-s \in \mathcal{O}$ is embedded in the plane with all of its vertices incident with the outer face. Clearly, $s \neq x$ and $s \neq u_{3}$, for otherwise $x$ or $u_{3}$ is apex in $G$, a contradiction. Also, $s \neq a$, since $\left(G \backslash x u_{3}\right)-a$ contains a $K_{2,3}$-subdivision (because $C\left(u_{1}, u_{2}\right) \neq \varnothing$, since $S_{1}$ is of type-one or type-two).

First, suppose that $w=u_{3}$. Then $u_{2} u_{3} \in E(C)$ (that is, $C\left(u_{2}, u_{3}\right)=\varnothing$ ). If $s=u_{2}$ (or by symmetry, if $s=u_{1}$ ), then in $\left(G \backslash x u_{3}\right)-s, \operatorname{deg}\left(u_{3}\right)=2$ and $\operatorname{deg}(x)=1$ hence edges $u_{3} u_{1}, u_{3} a$, and $x u_{1}$ are also incident with the outer face. Since $u_{3} u_{1}$ is a simple edge, by putting the edge $x u_{3}$ back in, we can embed $G-s$ so that all the vertices are still incident with the outer face, hence $G-s$ is outerplanar, a contradiction (see figure below). 


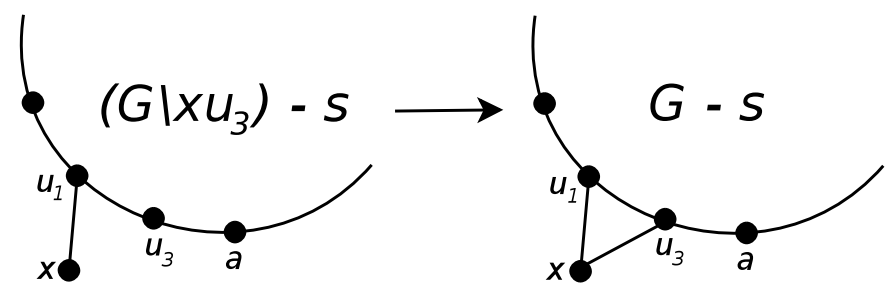

Therefore, $s \notin\left\{u_{1}, u_{2}, u_{3}, x, a\right\}$, so that $s \in C\left(u_{1}, u_{2}\right)$. Then, in $\left(G \backslash x u_{3}\right)-s$, $\operatorname{deg}(x)=2$, and so $\left(G \backslash x u_{3}\right)-s$ has an outerplanar embedding such that edges $x u_{1}$ and $x u_{2}$ are incident with the outer face. Also, note that $x, u_{1}, u_{3}, u_{2}$ is a 4 -cycle in $\left(G \backslash x u_{3}\right)-s$. Therefore, since $u_{1} u_{2} \notin E(G)$, we can put the edge $x u_{3}$ back in to obtain an embedding of $G-s$ in which all the vertices are still incident with the outer face, hence $G-s$ is outerplanar, a contradiction (see figure below).

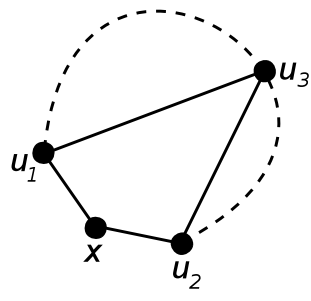

$\left(G \mid x u_{3}\right)-s$

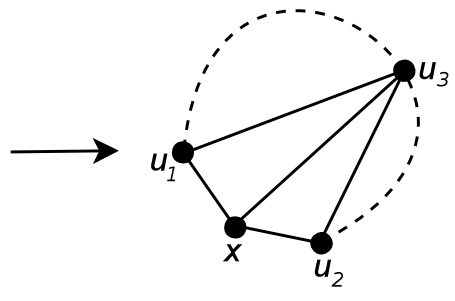

$G-S$

Therefore, $w \neq u_{3}$ and so $w \in C\left(u_{2}, u_{3}\right)$. Since $u_{2} w \in E(C)$, the only possible chords in $S_{2}$ have one endpoint at $u_{2}$ and the other in $C\left(w, u_{3}\right]$. Note that by Subcase 1 b hypothesis, $u_{3} a \notin E(G)$.

If $s=u_{2}$, then in $\left(G \backslash x u_{3}\right)-s, \operatorname{deg}\left(u_{3}\right)=2$ and $\operatorname{deg}(x)=1$, hence edges $u_{3} u_{1}, u_{3} w$, and $x u_{1}$ are incident with the outer face. Since $u_{3} u_{1}$ is a simple edge, by putting the edge $x u_{3}$ back in, we obtain an embedding of $G-s$ in which all the vertices are still incident with the outer face, hence $G-s$ is outerplanar, a contradiction.

Now suppose $s=u_{1}$. If $u_{2} u_{3}$ is a chord of $C$, then in $\left(G \backslash x u_{3}\right)-s, \operatorname{deg}\left(u_{3}\right)=2$ and $\operatorname{deg}(x)=1$, hence edges $u_{3} u_{2}, u_{3} w$, and $x u_{2}$ are incident with the outer face. Since $u_{3} u_{2}$ is a simple edge, by putting the edge $x u_{3}$ back in, we can embed $G-s$ so that all the vertices are still incident with the outer face, hence $G-s$ is outerplanar, 
a contradiction. Hence $u_{2} u_{3}$ is not a chord of $C$. If $G$ has a chord $c=u_{2} c_{1}$ with $c_{1} \in C\left(w, u_{3}\right)$, then choose $c_{1}$ closest to $u_{3}$, so that $c_{1} u_{3} \in E(C)$. And if there is no such chord, then let $c_{1}:=w$. Then, in $\left(G \backslash x u_{3}\right)-s, \operatorname{deg}(x)=1$, and $\operatorname{deg}\left(c_{1}\right)=3$, but $c_{1}$ is adjacent to $u_{3}$ with $\operatorname{deg}\left(u_{3}\right)=1$, hence edges $x u_{2}, u_{2} c_{1}$, and $c_{1} u_{3}$ are all incident with the outer face. Since $u_{2} c_{1}$ is a simple edge (even if $c_{1}=w$ ), by putting the edge $x u_{3}$ back in, we can embed $G-s$ so that all the vertices are still incident with the outer face, hence $G-s$ is outerplanar, a contradiction (see figure below).

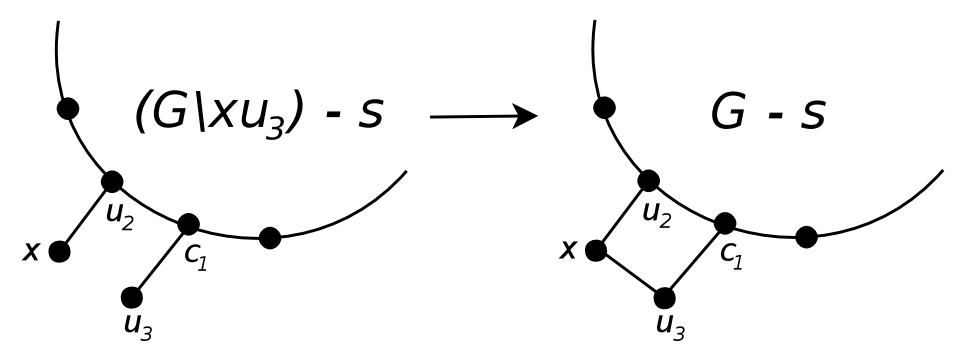

Similarly, if $s=w$, then $G$ has no chords with one endpoint at $u_{2}$ and the other in $C\left(w, u_{3}\right]$, for otherwise $\left(G \backslash x u_{3}\right)-s$ contains a $K_{2,3}$-subdivision (because $C\left(u_{1}, u_{2}\right) \neq \varnothing$, since $S_{1}$ is of type-one or type-two $)$. Hence, $C\left(w, u_{3}\right)=\varnothing$. Therefore, in $\left(G \backslash x u_{3}\right)-s, \operatorname{deg}(x)=2$ and $\operatorname{deg}\left(u_{3}\right)=1$, hence edges $x u_{1}$, and $u_{1} u_{3}$ are incident with the outer face. Since $x u_{1}$ is a simple edge, by putting the edge $x u_{3}$ back in, we can embed $G-s$ so that all the vertices are still incident with the outer face, hence $G-s$ is outerplanar, a contradiction.

Therefore, $s \notin\left\{u_{1}, u_{2}, u_{3}, x, a, w\right\}$, and so $s \in C\left(u_{1}, u_{2}\right)$ (by $(a)$ ). Again, if $G$ has a chord $c=u_{2} c_{1}$ with $c_{1} \in C\left(w, u_{3}\right)$, then choose $c_{1}$ closest to $u_{3}$, so that $c_{1} u_{3} \in E(G)$. And if there is no such chord, then let $c_{1}:=w$. Then, in $\left(G \backslash x u_{3}\right)-s$, $\operatorname{deg}(x)=2$, hence edges $x u_{2}$ and $x u_{1}$ are incident with the outer face. Also, note that $x, u_{1}, u_{3}, c_{1}, u_{2}$ is a 5 -cycle in $\left(G \backslash x u_{3}\right)-s$. Therefore, since $u_{1} u_{2} \notin E(G)$ and $u_{1} c_{1} \notin E(G)$ (by $(a)$ ), we can put the edge $x u_{3}$ back in (even if $u_{2} u_{3} \in E(G)$ ) to 
obtain an embedding of $G-s$ in which all the vertices are still incident with the outer face, hence $G-s$ is outerplanar, a contradiction (see figure below).

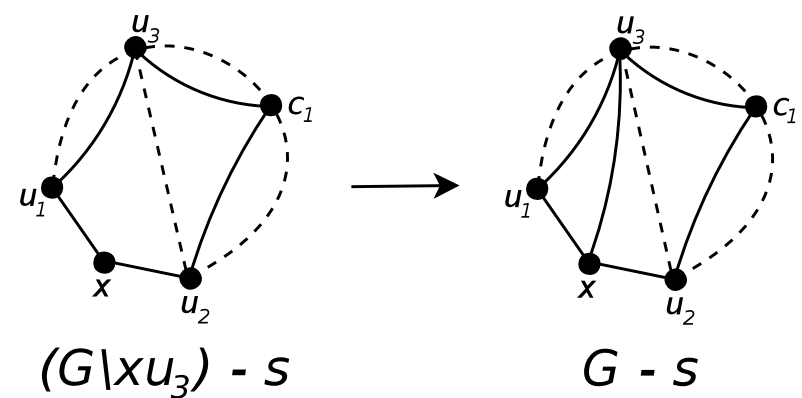

Case 1.2.2: $u_{1} u_{2} \in E(G)$

If all chords within $S_{1}$ have an endpoint at $u_{1}$ or all have an endpoint at $u_{2}$, then $u_{1}$, or $u_{2}$ respectively, is apex in $G$, a contradiction. Hence, there is a chord with both endpoints in $C\left(u_{1}, u_{2}\right)$. Let $c_{1} c_{2} \in E(G)$ be the innermost chord with $c_{1}, c_{2} \in C\left(u_{1}, u_{2}\right)$ (in the sense that there are no other chords with both endpoints in $\left.C\left[c_{1}, c_{2}\right]\right)$, and let $a_{1} \in N(a) \cap C\left(c_{1}, c_{2}\right)$.

Suppose that $a_{2} \neq a_{1}$ is another neighbor of $a$ in $C\left(c_{1}, c_{2}\right)$. Then by choice of $c_{1} c_{2}$, we have that $\operatorname{deg}\left(a_{1}\right)=3=\operatorname{deg}\left(a_{2}\right)$. Note that $a$ has no other neighbors in $C\left(u_{1}, u_{2}\right)$, for otherwise $G$ contains two disjoint $K$-graphs, a contradiction. Let $s$ be an apex vertex in $G \backslash a_{1} a_{2}$, and we assume that the graph $\left(G \backslash a_{1} a_{2}\right)-s \in \mathcal{O}$ is embedded in the plane with all of its vertices incident with the outer face. It is easy to see that $s=w$ (regardless of whether $w=u_{3}$ ), for otherwise: if $s \in\{a\} \cup C\left(u_{1}, u_{2}\right)$, then $\left(G \backslash a_{1} a_{2}\right)-s$ contains a $K_{4}$-subdivision; and if $u \in$ $\left\{u_{1}, u_{2}\right\} \cup C\left(w, u_{3}\right]$, then $\left(G \backslash a_{1} a_{2}\right)-s$ contains a $K_{2,3}$-subdivision. Therefore $s=w$, and hence the only neighbors of $a$ are $a_{1}, a_{2}$ and $w$ (because if $u_{1}$ or $u_{2}$ is a neighbor of $a$ then $\left(G \backslash a_{1} a_{2}\right)-s$ contains a $K_{2,3}$-subdivision). Then, in $\left(G \backslash a_{1} a_{2}\right)-s$, $\operatorname{deg}(a)=2$, hence edges $a a_{1}$ and $a a_{2}$ are incident with the outer face, and by putting 
the edge $a_{1} a_{2}$ back in, we obtain an embedding of $G-s$ in which all the vertices are still incident with the outer face, hence $G-s$ is outerplanar, a contradiction.

Hence, we have shown that $a_{1}$ is the only neighbor of $a$ in $C\left(c_{1}, c_{2}\right)$.

We now show furthermore that $N(a) \cap\left(C\left(u_{1}, c_{1}\right] \cup C\left[c_{2}, u_{2}\right)\right)=\varnothing$. For suppose otherwise, and let $c_{3} \in C\left(u_{1}, c_{1}\right]$ (the argument for $c_{3} \in C\left[c_{2}, u_{2}\right)$ is similar). Then $N(a) \cap C\left[c_{2}, u_{2}\right)=\varnothing$ (for otherwise $\left.G \geqslant_{m} 2 K_{4}\right)$. Let $s$ be an apex vertex in $G \backslash c_{1} a_{1}$. Then clearly $s \in\left\{u_{2}, w\right\}$. If $s=w$, then since $w$ is apex in $G \backslash c_{1} a_{1}$ we have that: $c_{3}=c_{1} ; N(a) \cap\left(C\left[u_{1}, c_{1}\right) \cup\left\{u_{2}\right\}\right)=\varnothing$; and $G$ does not have any chords with one endpoint at $u_{2}$ and the other in $C(w, u]$ (in the case that $w \neq u_{3}$ ). Therefore $w$ is apex in $G$, a contradiction. If $s=u_{2}$, then since $u_{2}$ is apex in $G \backslash c_{1} a_{1}$, it follows that $G$ has no chords with one endpoint in $C\left[u_{1}, c_{1}\right)$ and the other in $C\left[c_{2}, u_{2}\right)$. Hence all chords of $G$ have one endpoint at $c_{1}$ or at $u_{2}$. Therefore $u_{2}$ is apex in $G$, a contradiction.

Hence, we have shown that $N(a) \cap\left(C\left(u_{1}, a_{1}\right) \cup C\left(a_{1}, u_{2}\right)\right)=\varnothing$. Thus the only possible neighbors of $a$ are $u_{1}$ and $u_{2}$. In fact, at least one of them is a neighbor of $a$ since $\operatorname{deg}_{G}(a) \geqslant 3$. Let $s$ be an apex vertex in $G / a a_{1}$. Then clearly $s \in\left\{u_{1}, u_{2}\right\}$. Suppose that $s=u_{2}$ (the argument for $s=u_{1}$ is similar). Since $u_{2}$ is apex in $G / a a_{1}$, it follows that $G$ has no chords with one endpoint in $C\left[u_{1}, c_{1}\right)$ and the other in $C\left[c_{2}, u_{2}\right)$. Hence all chords of $G$ have one endpoint at $c_{1}$ or at $u_{2}$. Therefore $u_{2}$ is apex in $G$, a contradiction.

This concludes the proof of Subcase 1.2.2 and that of Case 1.

\subsection{Case 2}

In this case, we have that for every contractible edge $x y \in E(G), v_{x y}$ is an apex vertex in $G / x y$. 
The following simple Lemma provides a way of testing whether an edge in a 3-connected graph is contractible.

Lemma 4.6. Let $G$ be a 3-connected graph with edge $x y$. Then, G/xy is 3connected if and only if $G-\{x, y\}\left(=(G / x y)-v_{x y}\right)$ is 2-connected.

Proof. If $G / x y$ is 3 -connected, then clearly $(G / x y)-v_{x y}$ is 2-connected.

Now, suppose that $G-\{x, y\}\left(=(G / x y)-v_{x y}\right)$ is 2-connected and that $G / x y$ is not 3-connected, so that $G / x y$ has a 2 -cut. Since $G$ is 3 -connected, it follows that $v_{x y}$ is one of the vertices in that 2-cut (for otherwise, this 2-cut would also be a 2-cut in $G)$. Therefore, $(G / x y)-v_{x y}$ has a cut-vertex, a contradiction.

Let $x y$ be a contractible edge. Then, by the above Lemma, $(G / x y)-v_{x y} \in \mathcal{O}$ is 2-connected. Since $G$ is 3-connected it has a unique planar embedding. Restricting this embedding to $(G / x y)-v_{x y}$, we have that all the vertices of $(G / x y)-v_{x y}$ lie on a cycle $C$ and are incident with the outer face.

Let $x_{1}, x_{2}, \ldots, x_{m} \in V(C)(m \geqslant 2)$ be the neighbors of $x$ in the clockwise order around $C$. And let $y_{1}, y_{2}, \ldots, y_{n} \in V(C)(n \geqslant 2)$ be the neighbors of $y$ in the clockwise order around $C$. Note that $x_{i} \notin C\left(y_{1}, y_{n}\right)$ for all $i$ and $y_{j} \notin C\left(x_{1}, x_{m}\right)$ for all $j$, for otherwise $G$ would contain a $K_{3,3}$-minor. Also, note that possibly $x_{m}=y_{1}$ or $y_{n}=x_{1}$.

(a) The edges of $G$ are:

- edges of $C$;

- edges $x x_{i}$ for $i=1, \ldots, m$, and $y y_{j}$ for $j=1, \ldots, n$;

- chords of $C$, that is, edges not in $E(C)$ with both endpoints in $C$ (note that such edges are embedded in the interior of the disk bounded by $C$ );

- edge $x y$.

Just as in Case 1, it follows from (a) that: 
(b) The vertices of $C$ are either endpoints of chords or neighbors of $x$ or $y$.

(c) For every chord $c_{1} c_{2}$ in $G$ with $c_{1}<c_{2}$ (in the clockwise order restricted to the segment containing $\left.c_{1} c_{2}\right)$, there is a neighbor of $x$ or $y$ in $C\left(c_{1}, c_{2}\right)$.

Also, since neither $y$ nor $x$ is apex in $G$, it follows, respectively, that:

(d) $C\left(x_{1}, x_{m}\right) \neq \varnothing$ and $C\left(y_{1}, y_{n}\right) \neq \varnothing$.

Hence $G$ has the following general structure:

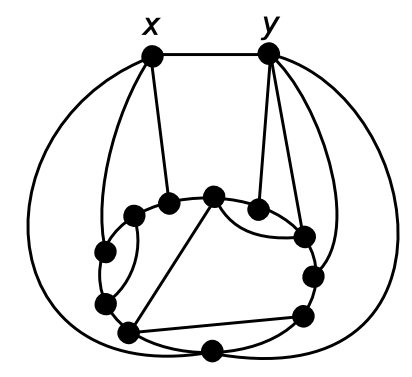

Finally, note that:

(e) If a vertex is not in $C\left[x_{m}, y_{1}\right] \cup C\left[y_{n}, x_{1}\right]$, then it is not apex.

This is because, neither $x$ nor $y$ is apex by $(d)$; and, $G-V\left(C\left(x_{1}, x_{m}\right)\right)$ and $G-V\left(C\left(y_{1}, y_{n}\right)\right)$ both contain $K_{4}$-subdivisions.

Before we proceed, we prove a Lemma regarding the structure of $G$.

Lemma 4.7. $G$ does not have a chord with both endpoints in $C\left[y_{n}, y_{1}\right]$. And by symmetry, the same statement holds for $C\left[x_{m}, x_{1}\right]$.

Proof. Let $c_{1} c_{2}$ be a chord of $G$ with both endpoints in $C\left[y_{n}, y_{1}\right]$. Without loss of generality, we may assume that $c_{1} c_{2}$ is the innermost such chord, in the sense that there are no other chords with both endpoints in $C\left[c_{1}, c_{2}\right]$. By $(c)$, it follows that $x$ has a neighbor $s$ in $C\left(c_{1}, c_{2}\right)$. Note that $x$ does not have another such neighbor $t$ in $C\left(c_{1}, c_{2}\right)$, for otherwise edge $s t$ is contractible (because $G-\{s, t\}$ is 2-connected), but $(G / s t)-v_{s t} \notin \mathcal{O}$ (because it contains a $K_{2,3}$-subdivision, since $\left.C\left(y_{1}, y_{n}\right) \neq \varnothing\right)$, violating the hypothesis of Case 2 . Therefore, the only vertex in $C\left(c_{1}, c_{2}\right)$ is $s$. But then, edge $x s$ is contractible (because $G-\{x, s\}$ is 2-connected), 
and $(G / x s)-v_{x s} \notin \mathcal{O}$ (because it contains a $K_{2,3}$-subdivision, since $\left.C\left(y_{1}, y_{n}\right) \neq \varnothing\right)$, again a contradiction.

The Lemma has the following consequence.

Corollary 4.8. The only chords in $G$ have one endpoint in $C\left(x_{1}, x_{m}\right)$ and the other in $C\left(y_{1}, y_{n}\right)$.

The following Lemma further tightens up the structure of $G$.

Lemma 4.9. There is exactly one vertex in $C\left(x_{1}, x_{m}\right)$ and exactly one in $C\left(y_{1}, y_{n}\right)$.

Proof. Suppose that $C\left(x_{1}, x_{m}\right)$ has two vertices $s$ and $t$. Then, by $(b)$ it follows that both $s$ and $t$ are neighbors of $x$, or endpoints of chords whose other endpoints lie in $C\left(y_{1}, y_{n}\right)$ by Corollary 4.8 , or both. Note that st is contractible (because $G-$ $\{s, t\}$ is 2-connected), and $(G / s t)-v_{s t} \notin \mathcal{O}$ (because it contains a $K_{4}$-subdivision, consisting of the cycle formed by edge $x x_{m}$, the clockwise path along $C$ from $x_{m}$ to $x_{1}$, and edge $x_{1} x$; and the three spokes from $y$ to this cycle), violating the hypothesis of Case 2.

With the structure of $G$ restricted by the above two Lemmas, we are ready to finish the proof. Let $s$ and $t$ be the unique vertices in $C\left(x_{1}, x_{m}\right)$ and $C\left(y_{1}, y_{n}\right)$, respectively. Note that $s t \in E(G)$, for otherwise any one of $x_{1}, x_{m}, y_{1}, y_{n}$ is apex, a contradiction. Also, it follows by Corollary 4.8 that $C\left(x_{m}, y_{1}\right)=\varnothing$ and $C\left(y_{n}, x_{1}\right)=\varnothing$.

If $x_{m} \neq y_{1}$ and $y_{n} \neq x_{1}$, then $G \geqslant_{m} Q$, a contradiction (see figure below).

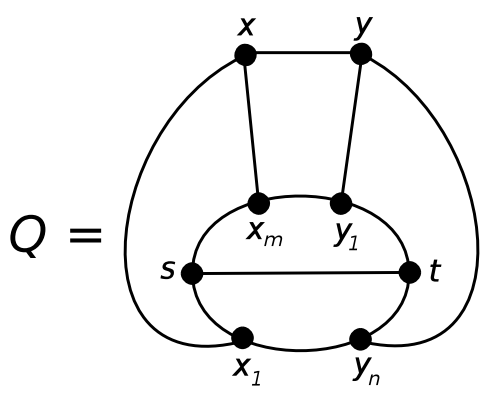


Hence, we have either the case that $x_{m} \neq y_{1}$ and $y_{n}=x_{1}$, or that $x_{m}=y_{1}$ and $y_{n}=x_{1}$. In either case, we cannot have that both $s x, t y \in E(G)$, for otherwise $G \geqslant_{m}$ Oct (see figure below).

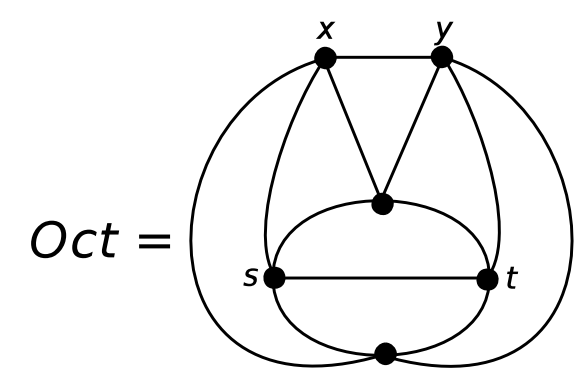

Hence, by symmetry, $s x \notin E(G)$, and it follows that $x_{m}$ is apex, a contradiction. This concludes the proof of Case 2, and also the connectivity-3 case. 


\section{References}

[1] I. Adler. Open problems related to computing obstruction sets. Pre-print, see www2.informatik.hu-berlin.de/ ${ }^{\sim}$ adler/publications/obstructions-open-problems.pdf.

[2] I. Adler, M. Grohe, S. Kreutzer. Computing excluded minors. Proceedings of the Nineteenth Annual ACM-SIAM Symposium on Discrete Algorithms, 641650, ACM, New York, 2008

[3] J.A. Bondy, U.S.R. Murty. Graph Theory, Springer, 2008.

[4] R. Diestel. Graph Theory, 2nd Edition, Springer-Verlag, New York, 2000.

[5] G. Ding, S. Dziobiak. Vertex-bipartition method for coloring minor-closed classes of graphs. Combin. Probab. Comput. 19 (4) (2010) 579-591.

[6] M. Fellows, M. Langston. On search, decision and the efficiency of polynomialtime algorithms (extended abstract). In 21st ACM Symposioum on Theory of Computing (1989), 501-512.

[7] R. Halin, H.A. Jung. Über minimalstrukturen von graphen, insbesondere von n-fach zusammenhängenden graphen, Math. Ann. 152 (1963), 75-94.

[8] H. Hadwiger. Über eine klassifikation der streckenkomplexe. Vierteljschr. Naturforsch. Ges. Zürich 88 (1943) 133-142.

[9] L. Jorgensen. Contraction to $K_{8}$. J. Graph Theory 18 (1994), 431-448.

[10] K. Kawarabayashi, B. Mohar. Some recent progress and applications in graph minor theory. Graphs Combin. 23 (1) (2007) 1-46.

[11] B. Mohar, C. Thomassen. Graphs on Surfaces, Johns Hopkins University Press, 2001.

[12] N. Robertson, D. Sanders, P. Seymour, R. Thomas. The four-colour theorem. J. Combin. Theory Ser. B 70 (1) (1997) 2-44.

[13] N. Robertson, P. Seymour. Graph Minors XIII. The disjoint paths problem. J. Comb. Theory Ser. B 63 (1995), 65-110.

[14] N. Robertson, P. Seymour. Graph minors XVI. Excluding a non-planar graph. J. Comb. Theory Ser. B 89 (2003) (1) 4376.

[15] N. Robertson, P. Seymour. Graph minors. XX. Wagners conjecture. J. Comb. Theory Ser. B 92 (2004), 325-357.

[16] N. Robertson, P. Seymour, R. Thomas. Hadwiger's conjecture for $K_{6}$-free graphs. Combinatorica 13 (3) (1993) 279-361. 
[17] N. Robertson, P. Seymour, R. Thomas. Sach's linkless embedding conjecture. J. Comb. Theory Ser. B 64 (1995), 185-227.

[18] S. Norin, R. Thomas. Configurations in large $t$-connected graphs. Pre-print.

[19] G.E. Turner. Personal communication.

[20] K. Wagner. Über eine eigenschaft der ebenen komplexe. Math. Ann. 114 (1) (1937) 570-590.

[21] L. Wargo. A characterization of $\alpha$-outerplanar graphs. J. Graph Theory 23 (1996), no. 1, 57-66.

[22] D. B. West. Introduction to Graph Theory, Prentice-Hall, Upper Saddle River, 1996. 


\section{Vita}

Stan Dziobiak was born on December 3, 1979 in Toruń, Poland. He finished his undergraduate studies at Cornell University in May 2002 majoring in computer science. He earned a master of engineering degree in computer science from Cornell University in May 2003. In August 2005 he came to Louisiana State University to pursue graduate studies in mathematics, where he earned a master of science degree in May 2007. He is currently a candidate for the degree of Doctor of Philosophy in mathematics, which will be awarded in August 2011. 